\title{
Semi-Direct Products of Lie Algebras and their Invariants
}

\author{
By
}

Dmitri I. PANYusheV*

\section{Contents}

$\S 1 . \quad$ Preliminaries

$\S 2$. Generic Stabilisers (centralisers) for the Adjoint Representation

$\S 3$. Generic Stabilisers for the Coadjoint Representation

$\S 4$. Semi-Direct Products of Lie Algebras and Modules of Covariants

$\S 5$. Generic Stabilisers and Rational Invariants for Semi-Direct Products

$\S 6$. Reductive Semi-Direct Products and their Polynomial Invariants

$\S 7 . \quad$ Takiff Lie Algebras and their Invariants

$\S 8$. The Null-Cone and its Irreducibility

$\S 9$. Isotropy Contractions and $\mathbb{Z}_{2}$-Contractions of Semisimple Lie Algebras

$\S 10$. Reductive Takiff Lie Algebras and their Representations

$\S 11$. On Invariants and Null-Cones for Generalised Takiff Lie Algebras

References

\section{Introduction}

The ground field $\mathbb{k}$ is algebraically closed and of characteristic zero. The goal of this paper is to extend the standard invariant-theoretic design, welldeveloped in the reductive case, to the setting of non-reductive group representations. This concerns the following notions and results: the existence of generic

\footnotetext{
Communicated by T. Kobayashi. Received December 5, 2006. Revised April 20, 2007. 2000 Mathematics Subject Classification(s): 14L30, 17B20, 22E46.

Supported in part by R.F.B.R. Grants 05-01-00988 and 06-01-72550.

*Independent Univ. Moscow, Bol'shoi Vlasevskii per. 11, 119002 Moscow, Russia.

e-mail: panyush@mccme.ru
} 
stabilisers and generic isotropy groups for (finite-dimensional rational) representations; structure of the fields and algebras of invariants; quotient morphisms and structure of their fibres. One of the main tools for obtaining non-reductive Lie algebras is the semi-direct product construction. There is a number of articles devoted to the study of the coadjoint representations of non-reductive Lie algebras; in particular, semi-direct products, see e.g. [31, 30, 32, 33, 41, 48]. In this article, we consider such algebras from a broader point of view. In particular, we found that the adjoint representation is an interesting object, too. Our main references for Invariant Theory are [5] and [46]. All algebraic groups are assumed to be linear.

If an algebraic group $A$ acts on an affine variety $X$, then $\mathbb{k}[X]^{A}$ stands for the algebra of $A$-invariant regular functions on $X$. If $\mathbb{k}[X]^{A}$ is finitely generated, then $X / / A:=\operatorname{Spec} \mathbb{k}[X]^{A}$, and the quotient morphism $\pi_{A}: X \rightarrow X / / A$ is the mapping associated with the embedding $\mathbb{k}[X]^{A} \hookrightarrow \mathbb{k}[X]$. If $\mathbb{k}[X]^{A}$ is polynomial, then the elements of any set of algebraically independent homogeneous generators will be referred to as basic invariants.

Let $G$ be a connected reductive algebraic group with Lie algebra $\mathfrak{g}$. Choose a Cartan subalgebra $\mathfrak{t} \subset \mathfrak{g}$ with the corresponding Weyl group W. The adjoint representation $(G: \mathfrak{g})$ has a number of good properties, some of which are listed below:

- The adjoint representation is self-dual, and $\mathfrak{t}$ is a generic stabiliser for it;

- The algebra of invariants $\mathbb{k}[\mathfrak{g}]^{G}$ is polynomial;

- the restriction homomorphism $\mathbb{k}[\mathfrak{g}] \rightarrow \mathbb{k}[\mathfrak{t}]$ induces the isomorphism $\mathbb{k}[\mathfrak{g}]^{G} \simeq \mathbb{k}[\mathfrak{t}]^{\mathrm{W}}$ (Chevalley's theorem);

- The quotient morphism $\pi_{G}: \mathfrak{g} \rightarrow \mathfrak{g} / / G$ is equidimensional and the fibre of the origin, $\mathcal{N}:=\pi_{G}^{-1}\left(\pi_{G}(0)\right)$, is an irreducible complete intersection. The ideal of $\mathcal{N}$ in $\mathbb{k}[\mathfrak{g}]$ is generated by the basic invariants;

- $\mathcal{N}$ is the union of finitely many $G$-orbits.

Each of these properties may fail if $\mathfrak{g}$ is replaced with an arbitrary algebraic Lie algebra $\mathfrak{q}$. In particular, one have to distinguish the adjoint and coadjoint representations of $\mathfrak{q}$. As usual, ad (resp. $\mathrm{ad}^{*}$ ) stands for the adjoint (resp. coadjoint) representation. Write $Q$ for a connected group with Lie algebra $\mathfrak{q}$.

First, we consider the problem of existence of generic stabilisers for ad and $\mathrm{ad}^{*}$. (See $\S 1$ for precise definitions). It turns out that if $(\mathfrak{q}$, ad $)$ has a generic stabiliser, say $\mathfrak{h}$, then $\mathfrak{h}$ is commutative and $\mathfrak{n}_{\mathfrak{q}}(\mathfrak{h})=\mathfrak{h}$. This yields a Chevalley-type theorem for the fields of invariants: $\mathbb{k}(\mathfrak{q})^{Q} \simeq \mathbb{k}(\mathfrak{h})^{W}$, where $W=N_{Q}(\mathfrak{h}) / Z_{Q}(\mathfrak{h})$ is finite. We also notice that $(\mathfrak{q}$, ad $)$ has a generic stabiliser 
if and only if the Cartan subalgebras of $\mathfrak{q}$ are commutative. If $\left(\mathfrak{q}, \mathrm{ad}^{*}\right)$ has a generic stabiliser, say $\mathfrak{h}$, then $\mathfrak{h}$ is commutative, $\operatorname{dim} N_{Q}(\mathfrak{h})=\operatorname{dim}\left(\mathfrak{q}^{*}\right)^{\mathfrak{h}}$, and $\mathbb{k}\left(\mathfrak{q}^{*}\right)^{Q} \simeq \mathbb{k}\left(\left(\mathfrak{q}^{*}\right)^{\mathfrak{h}}\right)^{N_{Q}(\mathfrak{h})}$. But unlike the adjoint case, the action $\left(N_{Q}(\mathfrak{h}):\left(\mathfrak{q}^{*}\right)^{\mathfrak{h}}\right)$ does not necessarily reduce to a finite group action. We prove that under a natural constraint the representation of the identity component of $N_{Q}(\mathfrak{h})$ on $\left(\mathfrak{q}^{*}\right)^{\mathfrak{h}}$ is the coadjoint representation.

Our main efforts are connected with the following situation. Suppose that $(\mathfrak{q}, \operatorname{ad})$ or $\left(\mathfrak{q}, \mathrm{ad}^{*}\right)$ has some of the above good properties and $V$ is a (finitedimensional rational) $Q$-module. Form the Lie algebra $\mathfrak{q} \ltimes V$. It is the semidirect product of $\mathfrak{q}$ and $V, V$ being a commutative ideal in it. The corresponding connected algebraic group is $Q \ltimes V$. (See Section 4 for the details.) Then we want to realise to which extent those good properties are preserved under this procedure. This surely depends on $V$, and we are essentially interested in two cases:

(a) $\mathfrak{q}$ is arbitrary and $V=\mathfrak{q}$ or $\mathfrak{q}^{*}$ (the adjoint or coadjoint $\mathfrak{q}$-module);

(b) $\mathfrak{q}=\mathfrak{g}$ is reductive and $V$ is an arbitrary $G$-module.

For (a), we prove that if ( $\mathfrak{q}$, ad ) has a generic stabiliser, then so do $(\mathfrak{q} \ltimes \mathfrak{q}$, ad ) and $\left(\mathfrak{q} \ltimes \mathfrak{q}^{*}\right.$, ad $)$. Furthermore, the passages $\mathfrak{q} \rightsquigarrow \mathfrak{q} \ltimes \mathfrak{q}$ and $\mathfrak{q} \rightsquigarrow \mathfrak{q} \ltimes \mathfrak{q}^{*}$ does not affect the generalised Weyl group $W$, and both fields $\mathbb{k}(\mathfrak{q} \ltimes \mathfrak{q})^{Q \ltimes \mathfrak{q}}$ and $\mathbb{k}\left(\mathfrak{q} \ltimes \mathfrak{q}^{*}\right)^{Q \ltimes \mathfrak{q}^{*}}$ are purely transcendental extensions of $\mathbb{k}(\mathfrak{q})^{Q}$. It is also true that if $\left(\mathfrak{q}, \operatorname{ad}^{*}\right)$ has a generic stabiliser, then so does $\left(\mathfrak{q} \ltimes \mathfrak{q}, \operatorname{ad}^{*}\right)$.

For (b), we prove that $(\mathfrak{g} \ltimes V$, ad ) always has a generic stabiliser. But this is not the case for ad*. Recall that any $\mathfrak{g}$-module $V$ has a generic stabiliser. The following result seems to be quite unexpected. Suppose generic $G$-orbits in $V$ are closed (i.e., the action $(G: V)$ is stable), then $\left(\mathfrak{g} \ltimes V\right.$, ad $\left.{ }^{*}\right)$ has a generic stabiliser if and only if $V$ is a polar $G$-module in the sense of [11]. The assumption of stability is relatively harmless, since there are only finitely many $G$-modules without that property. On the other hand, the hypothesis of being polar is quite restrictive, because for any $G$ there are only finitely many polar representations.

One of our main observations is that there are surprisingly many nonreductive Lie algebras $\mathfrak{a}$ and $\mathfrak{a}$-modules $M$ such that $\mathbb{k}[M]^{A}$ is a polynomial algebra. Furthermore, the basic invariants of $\mathbb{k}[M]^{A}$ can explicitly be constructed using certain modules of covariants. This concerns the following cases:

- If $\mathfrak{g}$ is reductive and $V$ is an arbitrary $\mathfrak{g}$-module, then one takes $\mathfrak{a}=M=$ $\mathfrak{g} \ltimes V ;$

- If the action $(Q: V)$ satisfies some good properties, then one takes $\mathfrak{a}=$ $\mathfrak{q} \ltimes \mathfrak{q}$ and $M=V \ltimes V$. Furthermore, the passage $(\mathfrak{q}, V) \mapsto(\hat{\mathfrak{q}}=\mathfrak{q} \ltimes \mathfrak{q}, \hat{V}=V \ltimes V)$ 
can be iterated.

The precise statements are given below.

0.1 Theorem. Let $V$ be an arbitrary $G$-module. Set $\mathfrak{q}=\mathfrak{g} \ltimes V, Q=$ $G \ltimes V$, and $m=\operatorname{dim} V^{\mathfrak{t}}$. Notice that $1 \ltimes V$ is a commutative normal subgroup of $Q$ (in fact, the unipotent radical of $Q$ ). Then

(i) $\mathbb{k}[\mathfrak{q}]^{1 \ltimes V}$ is a polynomial algebra of Krull dimension $\operatorname{dim} \mathfrak{g}+m$. It is freely generated by the coordinates on $\mathfrak{g}$ and the functions $\widehat{F}_{i}, i=1, \ldots, m$, associated with covariants of type $V^{*}$.

(ii) $\mathbb{k}[\mathfrak{q}]^{Q}$ is a polynomial algebra of Krull dimension $\operatorname{dim} \mathfrak{t}+m$. It is freely generated by the basic invariants of $\mathbb{k}[\mathfrak{g}]^{G}$ and the same functions $\widehat{F}_{i}, i=$ $1, \ldots, m$.

(iii) $\max \operatorname{dim}_{x \in \mathfrak{q}} Q \cdot x=\operatorname{dim} \mathfrak{q}-\operatorname{dim} \mathfrak{q} / / Q$;

(iv) If $\pi: \mathfrak{q} \rightarrow \mathfrak{q} / / Q$ is the quotient morphism and $\Omega:=\left\{x \in \mathfrak{q} \mid d \pi_{x}\right.$ is onto $\}$, then $\mathfrak{q} \backslash \Omega$ contains no divisors.

Given a $\mathfrak{q}$-module $V$, the space $V \times V$ can be regarded as $\mathfrak{q} \ltimes \mathfrak{q}$-module in a very natural way. Write $\hat{V}$ or $V \ltimes V$ for this module.

0.2 Theorem. Suppose the action $(Q: V)$ satisfies the following conditions:

(1) $\mathbb{k}[V]^{Q}$ is a polynomial algebra;

(2) $\max \operatorname{dim}_{v \in V} Q \cdot v=\operatorname{dim} V-\operatorname{dim} V / / Q$;

(3) If $\pi_{Q}: V \rightarrow V / / Q$ is the quotient morphism and $\Omega:=\{v \in V \mid$ $\left(d \pi_{Q}\right)_{v}$ is onto $\}$, then $V \backslash \Omega$ contains no divisors.

Set $\hat{\mathfrak{q}}=\mathfrak{q} \ltimes \mathfrak{q}$ and $\hat{Q}=Q \ltimes \mathfrak{q}$. Then

(i) $\mathbb{k}[\hat{V}]^{1 \ltimes \mathfrak{q}}$ is a polynomial algebra of Krull dimension $\operatorname{dim} V+\operatorname{dim} V / / Q$, which is generated by the coordinates on the first factor of $\hat{V}$ and the polynomials $\widehat{F}_{1}, \ldots, \widehat{F}_{m}$ associated with the differentials of basic invariants in $\mathbb{k}[V]^{Q}$;

(ii) $\mathbb{k}[\hat{V}]^{\hat{Q}}$ is a polynomial algebra of Krull dimension $2 \operatorname{dim} V / / Q$, which is freely generated by the basic invariants of $\mathbb{k}[V]^{Q}$ and the same functions $\widehat{F}_{i}, i=1, \ldots, m$.

(iii) The $\hat{Q}$-module $\hat{V}$ satisfies conditions (1)-(3), too. 
Since the adjoint representation of a reductive Lie algebra $\mathfrak{g}$ satisfies the above properties (1)-(3), one may begin with $\mathfrak{q}=\mathfrak{g}=V$, and iterate the procedure ad infinitum. For the adjoint representation of a semisimple Lie algebra, the assertion in part (ii) is due to Takiff [40]. For this reason Lie algebras of the form $\mathfrak{q} \ltimes \mathfrak{q}$ are called Takiff (Lie) algebras. We will also say that the $\hat{\mathfrak{q}}$-module $\hat{V}$ is the Takiffisation of the $\mathfrak{q}$-module $V$. But $(\mathfrak{g}$, ad $)$ is not the only possible point of departure for the infinite iteration process. In view of Theorem 0.1, the algebras $\mathfrak{q}=\mathfrak{g} \ltimes V$ and their adjoint representations can also be used as initial bricks for the Takiffisation procedure.

If $\mathbb{k}[V]^{Q}$ is polynomial, then it is natural to study the fibres of the quotient morphism $\pi_{Q}$. The null-cone, $\mathcal{N}(V)=\pi_{Q}^{-1}\left(\pi_{Q}(0)\right)$, is the most important fibre. For instance, $\mathbb{k}[V]$ is a free $\mathbb{k}[V]^{Q}$-module if and only $\operatorname{dim} \mathcal{N}(V)=\operatorname{dim} V-$ $\operatorname{dim} V / / Q$, i.e., $\pi_{Q}$ is equidimensional. We consider properties of null-cones arising in the context of semi-direct products and their representations.

For $\mathfrak{q}=\mathfrak{g} \ltimes V$, as in Theorem 0.1 , a necessary and sufficient condition for the equidimensionality of $\pi_{Q}$ is stated in terms of a stratification of $\mathcal{N}$ determined by the covariants on $\mathfrak{g}$ of type $V^{*}$. Using this stratification and some technique from [27] and [22], we prove the following:

If $\mathcal{N}(\mathfrak{q})$ is irreducible, then (i) $\pi_{Q}$ is equidimensional; (ii) the morphism $\kappa: \mathfrak{q} \rightarrow \mathfrak{q}$ defined by $\kappa(x, v)=(x, x \cdot v), x \in \mathfrak{g}, v \in V$, has the property that the closure of $\operatorname{Im}(\kappa)$ is a factorial complete intersection and its ideal in $\mathbb{k}[\mathfrak{q}]$ is generated by the polynomials $\widehat{F}_{i}, i=1, \ldots, m$, mentioned in Theorem 0.1 . This is a generalisation of [22, Prop. 2.4]. Similar results hold for the Takiffisation of $G$-modules $V$ having good properties, as in Theorem 0.2. In this case, conditions of equidimensionality for $\pi_{\hat{G}}: \hat{V} \rightarrow \hat{V} / / \hat{G}$ are stated in terms of a stratification of $\mathcal{N}(V)$ determined by the covariants on $V$ of type $V^{*}$. See $\S 8$ for the details.

In general, it is difficult to deal with the stratifications of $\mathcal{N}$ and $\mathcal{N}(V)$, but, for isotropy contractions and $\mathbb{Z}_{2}$-contractions of reductive Lie algebras, explicit results can be obtained. Let $\mathfrak{h}$ be a reductive subalgebra of $\mathfrak{g}$ and $\mathfrak{g}=\mathfrak{h} \oplus \mathfrak{m}$ a direct sum of $\mathfrak{h}$-modules. Then $\mathfrak{h} \ltimes \mathfrak{m}$ is called an isotropy contraction of $\mathfrak{g}$. If $\mathfrak{g}=\mathfrak{h} \oplus \mathfrak{m}$ is a $\mathbb{Z}_{2}$-grading, then we say about a $\mathbb{Z}_{2}$-contraction. (The word "contraction" can be understood in the usual sense of deformation theory of Lie algebras.) Semi-direct products occurring in this way have some interesting properties. As a sample, we mention the following useful fact: ind $(\mathfrak{h} \ltimes \mathfrak{m})=$ ind $\mathfrak{g}+2 c(G / H)$, where ind (.) is the index of a Lie algebra and $c($.$) is the$ complexity of a homogeneous space. In particular, ind $(\mathfrak{h} \ltimes \mathfrak{m})=$ ind $\mathfrak{g}$ if and only if $H$ is a spherical subgroup of $G$. 
Our main results on the equidimensionality of quotient morphisms and irreducibility of null-cones are related to the $\mathbb{Z}_{2}$-contractions of simple Lie algebras. Given a $\mathbb{Z}_{2}$-grading $\mathfrak{g}=\mathfrak{g}_{0} \oplus \mathfrak{g}_{1}$, Theorem 0.1 applies to the semi-direct product $\mathfrak{k}=\mathfrak{g}_{0} \ltimes \mathfrak{g}_{1}$, so that $\mathbb{k}[\mathfrak{k}]^{K}$ is a polynomial algebra of Krull dimension rk $\mathfrak{g}$. Using the classification of $\mathbb{Z}_{2}$-gradings, we prove that $\mathcal{N}(\mathfrak{k})$ is irreducible. Therefore the good properties discussed in a preceding paragraph hold for the morphism $\kappa: \mathfrak{k} \rightarrow \mathfrak{k}, \kappa\left(x_{0}, x_{1}\right)=\left(x_{0},\left[x_{0}, x_{1}\right]\right)$. Our proof of irreducibility of $\mathcal{N}(\mathfrak{k})$ basically reduces to the verification of certain inequality for the nilpotent $G_{0}$-orbits in $\mathfrak{g}_{0}$. Actually, we notice that one may prove a stronger constraint (cf. inequalities (9.8) and (9.9)). This leads to the following curious result: Consider $\tilde{\mathfrak{k}}=\mathfrak{g}_{0} \ltimes\left(\mathfrak{g}_{1} \oplus \mathfrak{g}_{1}\right)$. (In view of Theorem $0.1, \mathbb{k}[\tilde{\mathfrak{k}}]^{\tilde{K}}$ is polynomial.) Then $\pi_{\tilde{K}}$ is still equidimensional, although $\mathcal{N}(\tilde{\mathfrak{k}})$ can already be reducible.

To discuss similar results for the Takiffisation of $\mathfrak{q}$-modules, i.e., $\hat{\mathfrak{q}}$-modules $\hat{V}$, one has to impose more constraints on $V$. We also assume below that $\mathfrak{q}=\mathfrak{g}$ is reductive.

0.3 Theorem. Suppose the $G$-module $V$ satisfies conditions (1)-(3) of Theorem 0.2 and also the following two conditions:

(4) $\mathcal{N}(V):=\pi_{G}^{-1}\left(\pi_{G}(0)\right)$ consists of finitely many $G$-orbits;

(5) $\mathcal{N}(V)$ is irreducible and has only rational singularities.

For $\pi_{\hat{G}}: \hat{V} \rightarrow \hat{V} / / \hat{G}$ and $\mathcal{N}(\hat{V})=\pi_{\hat{G}}^{-1}\left(\pi_{\hat{G}}(0)\right)$, we then have, in addition to the conclusions of Theorem 0.2 ,

(i) $\mathcal{N}(\hat{V})$ is an irreducible complete intersection and the ideal of $\mathcal{N}(\hat{V})$ in $\mathbb{k}[\hat{V}]$ is generated by the basic invariants in $\mathbb{k}[\hat{V}]^{\hat{G}}$;

(ii) $\pi_{\hat{G}}$ is equidimensional and $\mathbb{k}[\hat{V}]$ is a free $\mathbb{k}[\hat{V}]^{\hat{G}}$-module.

For $G$ semisimple, conditions (2) and (3) are satisfied for all $V$, therefore the most essential conditions are (4) and (5). The main point here is to prove the irreducibility. The crucial step in proving this theorem is the use of the Goto-Watanabe inequality [25, Theorem 2'] which relates the dimension and embedding dimension of the local rings that are complete intersections with only rational singularities, see $\S 10$. (We refer to [18] for the definition of rational singularities.) For $V=\mathfrak{g}$, the idea of using that inequality is due to M. Brion. The irreducibility of $\mathcal{N}(\hat{\mathfrak{g}})$ was first proved by F. Geoffriau [16] via case-bycase checking. Then, applying the Goto-Watanabe inequality, Brion found a conceptual proof of Geoffriau's result [6]. Our observation is that Brion's idea applies in a slightly more general setting of the Takiffisation of representations $(G: V)$ satisfying conditions (1)-(5). 
The irreducibility of $\mathcal{N}(\hat{\mathfrak{g}})$ is equivalent to that a certain inequality holds for all non-regular nilpotent elements (orbits). Here is it:

$$
\operatorname{dim} \mathfrak{z}_{\mathfrak{g}}(x)+\operatorname{rk}\left(d \pi_{G}\right)_{x}>2 \operatorname{rkg} \quad \text { if } x \in \mathcal{N} \backslash \mathcal{N}^{\text {reg }} .
$$

Using case-by-case checking, we prove a stronger inequality

$$
\operatorname{dim} \mathfrak{z}_{\mathfrak{g}}(x)+2 \operatorname{rk}\left(\mathrm{d} \pi_{G}\right)_{x}-3 \operatorname{rkg} \mathfrak{g} \geqslant 0 \text { for all } x \in \mathcal{N} .
$$

It seems that the last inequality is more fundamental, because it is stated more uniformly, can be written in different equivalent forms, and has geometric applications. For instance, if $\mathfrak{g}=\mathfrak{g}_{0} \oplus \mathfrak{g}_{1}$ is a $\mathbb{Z}_{2}$-grading of maximal rank and $\hat{\mathfrak{g}}_{1}=\mathfrak{g}_{1} \ltimes \mathfrak{g}_{1}$, then the equidimensionality of $\pi_{\hat{G}_{0}}: \hat{\mathfrak{g}}_{1} \rightarrow \hat{\mathfrak{g}}_{1} / / \hat{G}_{0}$ is essentially equivalent to the last inequality. This result cannot be deduced from Theorem 0.3 , because $\mathcal{N}\left(\mathfrak{g}_{1}\right)$ is not normal. Furthermore, $\mathcal{N}\left(\hat{\mathfrak{g}}_{1}\right)$ can be reducible.

Our methods also work for generalised Takiff algebras introduced in [33]. The vector space $\mathfrak{q}_{\infty}:=\mathfrak{q} \otimes \mathbb{k}[\mathrm{T}]$ has a natural Lie algebra structure such that $\left[x \otimes \mathrm{T}^{l}, y \otimes \mathrm{T}^{k}\right]=[x, y] \otimes \mathrm{T}^{l+k}$. Then $\mathfrak{q}_{\geqslant(n+1)}=\bigoplus_{j \geqslant n+1} \mathfrak{q} \otimes \mathrm{T}^{j}$ is an ideal of $\mathfrak{q}_{\infty}$, and the respective quotient is a generalised Takiff Lie algebra, denoted $\mathfrak{q}\langle n\rangle$. Write $Q\langle n\rangle$ for the corresponding connected group. Clearly, $\operatorname{dim} \mathfrak{q}\langle n\rangle=(n+1) \operatorname{dim} \mathfrak{q}$ and $\mathfrak{q}\langle 1\rangle \simeq \mathfrak{q} \ltimes \mathfrak{q}$. We prove that if $(Q: \mathfrak{q})$ satisfies conditions (1)-(3) of Theorem 0.2, then the similar conclusions hold for the adjoint action $(Q\langle n\rangle: \mathfrak{q}\langle n\rangle)$. In particular, $\mathbb{k}[\mathfrak{q}\langle n\rangle]^{Q\langle n\rangle}$ is a polynomial algebra of Krull dimension $(n+1) \operatorname{dim} \mathfrak{q} / / Q$.

For $\mathfrak{q}=\mathfrak{g}$ semisimple, our methods enable us to deduce the equidimensionality of $\pi_{G\langle 2\rangle}: \mathfrak{g}\langle 2\rangle \rightarrow \mathfrak{g}\langle 2\rangle / / G\langle 2\rangle$ from the same fact related to the semi-direct product $\mathfrak{g} \ltimes(\mathfrak{g} \oplus \mathfrak{g})$. However, it was shown by Eisenbud and Frenkel that $\pi_{G\langle n\rangle}: \mathfrak{g}\langle n\rangle \rightarrow \mathfrak{g}\langle n\rangle / / G\langle n\rangle$ is equidimensional for any $n$, see [24, Appendix]. Their proof exploits the interpretation of $\mathcal{N}(\mathfrak{g}\langle n\rangle)$ as a jet scheme and uses the deep result of Mustaţă concerning the irreducibility of jet schemes [24, Theorem 3.3].

\section{$\S 1$. Preliminaries}

Algebraic groups are denoted by capital Latin letters and their Lie algebras are denoted by the corresponding lower-case Gothic letters. The identity component of an algebraic group $Q$ is denoted by $Q^{\circ}$.

Let $Q$ be an affine algebraic group acting regularly on an irreducible variety $X$. Then $Q_{x}$ stands for the isotropy group of $x \in X$. Likewise, the stabiliser of $x$ in $\mathfrak{q}=$ Lie $Q$ is denoted by $\mathfrak{q}_{x}$. We write $\mathbb{k}[X]^{Q}\left(\right.$ resp. $\left.\mathbb{k}(X)^{Q}\right)$ for the algebra 
of regular (resp. field of rational) $Q$-invariants on $X$. A celebrated theorem of M. Rosenlicht says that there is a dense open $Q$-stable subset $\tilde{\Omega} \subset X$ such that $\mathbb{k}(X)^{H}$ separates the $Q$-orbits in $\tilde{\Omega}$, see e.g. $[5,1.6]$, [46, 2.3]. In particular, $\operatorname{trdeg} \mathbb{k}(X)^{Q}=\operatorname{dim} X-\max \operatorname{dim}_{x \in X} Q \cdot x$. We will use Rosenlicht's theorem in the following equivalent form:

1.1 Theorem. Let $\mathbb{F}$ be a subfield of $\mathbb{k}(X)^{Q}$. Then $\mathbb{F}=\mathbb{k}(X)^{Q}$ if and only if $\mathbb{F}$ separates the $Q$-orbits in a dense open subset of $X$.

We say that the action $(Q: X)$ has a generic stabiliser, if there exists a dense open subset $\Omega \subset X$ such that all stabilisers $\mathfrak{q}_{\xi}, \xi \in \Omega$, are $Q$-conjugate. Then each of the subalgebras $\mathfrak{q}_{\xi}, \xi \in \Omega$, is called a generic stabiliser. The points of such an $\Omega$ are said to be generic. Likewise, one defines a generic isotropy group, which is a subgroup of $Q$. Clearly, the existence of a generic isotropy group implies that of a generic stabiliser. That the converse is also true is proved by Richardson $[34, \S 4]$. The reader is also referred to $[46, \S 7]$ for a thorough discussion of generic stabilisers. If $Y \subset X$ is irreducible, then $Y^{r e g}:=\{y \in$ $\left.Y \mid \operatorname{dim} Q \cdot y=\max _{z \in Y} \operatorname{dim} Q \cdot z\right\}$. It is a dense open subset of $Y$. The points of $Y^{\text {reg }}$ are said to be regular. Of course, these notions depend on $\mathfrak{q}$. If we wish to make this dependence explicit, we speak about $\mathfrak{q}$-generic or $\mathfrak{q}$-regular points. Since $X^{r e g}$ is dense in $X$, all generic points (if they do exist) are regular. The converse is however not true.

If $Q$ is reductive and $X$ is smooth, then $(Q: X)$ always has a generic stabiliser [34]. One of our goals is to study existence of generic stabilisers in case of non-reductive $Q$. Specifically, we consider the adjoint and coadjoint representations of $Q$. To this end, we recall some standard invariant-theoretic techniques and a criterion for the existence of generic stabilisers.

Let $\rho: Q \rightarrow G L(V)$ be a finite-dimensional rational representation of $Q$ and $\bar{\rho}: \mathfrak{q} \rightarrow \mathfrak{g l}(V)$ the corresponding representation of $\mathfrak{q}$. For $s \in Q$ and $v \in V$, we usually write $s \cdot v$ in place of $\rho(s) v$. Similarly, $x \cdot v$ is a substitute for $\bar{\rho}(x) v$, $x \in \mathfrak{q}$. (But for the adjoint representation, the standard bracket notation is used.) It should be clear from the context which meaning of '. ' is meant. Given $v \in V$, consider

$$
U=V^{\mathfrak{q}_{v}}=\left\{y \in V \mid \mathfrak{q}_{v} \cdot y=0\right\},
$$

the fixed point space of $\mathfrak{q}_{v}$. Associated to $U \subset V$, there are two subgroups of Q:

$$
\mathbf{N}(U)=\{s \in Q \mid s \cdot U \subset U\}, \quad \mathrm{Z}(U)=\{s \in Q \mid s \cdot u=u \text { for all } u \in U\} .
$$

The following is well known and easy. 


\subsection{Lemma.}

(i) $\operatorname{Lie} \mathrm{Z}(U)=\mathfrak{q}_{v}$ and $\mathrm{Z}(U)$ is a normal subgroup of $\mathrm{N}(U)$;

(ii) $\mathrm{N}(U)=N_{Q}(\mathrm{Z}(U))=N_{Q}\left(\mathfrak{q}_{v}\right)$.

It is not necessarily the case that $\mathbf{Z}(U)$ is connected; however, $\mathbf{Z}(U)$ and $\mathbf{Z}(U)^{o}$ have the same normaliser in $Q$.

1.3 Lemma. If $y \in U^{r e g}$ (i.e., $\left.\mathfrak{q}_{y}=\mathfrak{q}_{v}\right)$, then $Q \cdot y \cap U=\mathrm{N}(U) \cdot y$ and $\mathfrak{q} \cdot y \cap U=\mathfrak{n}_{\mathfrak{q}}\left(\mathfrak{q}_{v}\right) \cdot y$.

Proof. 1. Suppose $s \cdot y \in U$ for some $s \in Q$. Then $\mathfrak{q}_{s \cdot y}=\mathfrak{q}_{v}=\mathfrak{q}_{y}$. Hence $s \in N_{Q}\left(\mathfrak{q}_{v}\right)$, and we refer to Lemma 1.2 .

2. Suppose $s \cdot y \in U$ for some $s \in \mathfrak{q}$. Then $0=\mathfrak{q}_{v}(s \cdot y)=\left[\mathfrak{q}_{v}, s\right] \cdot y$. Hence $\left[\mathfrak{q}_{v}, s\right] \subset \mathfrak{q}_{y}=\mathfrak{q}_{v}$.

Set $Y=\overline{Q \cdot U}$. It is a $Q$-stable irreducible subvariety of $V$.

1.4 Proposition. The restriction homomorphism $\left.(f \in \mathbb{k}(Y)) \mapsto f\right|_{U}$ yields an isomorphism $\mathbb{k}(Y)^{Q} \stackrel{\sim}{\longrightarrow} \mathbb{k}(U)^{\mathrm{N}(U)}=\mathbb{k}(U)^{\mathrm{N}(U) / \mathrm{Z}(U)}$.

Proof. This follows from the first equality in Lemma 1.3 and Rosenlicht's theorem.

1.5 Example. Let $G$ be a semisimple algebraic group with Lie algebra $\mathfrak{g}$, and $v=e \in \mathfrak{g}$ a nilpotent element. Then $\mathfrak{g}_{e}=\mathfrak{z}_{\mathfrak{g}}(e)$ is the centraliser of $e$ and $U=\left\{x \in \mathfrak{g} \mid\left[x, \mathfrak{z}_{\mathfrak{g}}(e)\right]=0\right\}=: \mathfrak{d}_{\mathfrak{g}}(e)$ is the centre of $\mathfrak{z}_{\mathfrak{g}}(e)$. Here $\mathrm{N}(U)=N_{G}\left(\mathfrak{z}_{\mathfrak{g}}(e)\right)$ is the normaliser of $\mathfrak{z}_{\mathfrak{g}}(e)$ in $G$. Letting $Y=\overline{G \cdot \mathfrak{d}_{\mathfrak{g}}(e)}$, we obtain an isomorphism

$$
\mathbb{k}(Y)^{G} \simeq \mathbb{k}\left(\mathfrak{d}_{\mathfrak{g}}(e)\right)^{N_{G}\left(\mathfrak{z}_{\mathfrak{g}}(e)\right)}
$$

It is known that $\mathfrak{d}_{\mathfrak{g}}(e)$ contains no semisimple elements [9], so that $Y$ is the closure of a nilpotent orbit and hence $\mathbb{k}(Y)^{G}=\mathbb{k}$. It follows that $N_{G}\left(\mathfrak{z}_{\mathfrak{g}}(e)\right)$ has a dense orbit in $\mathfrak{d}_{\mathfrak{g}}(e)$. This fact was already noticed in [30, $\left.\S 4\right]$. Actually, the dense $G$-orbit in $Y$ is just $G \cdot e$.

Clearly, if $\overline{Q \cdot U}=V$, then $(Q: V)$ has a generic stabiliser and $v$ is a generic point. A general criterion for this to happen is proved in $[14, \S 1]$. For future reference, we recall it here. 
1.6 Lemma (Elashvili). Let $v \in V$ be an arbitrary point. Then $Q \cdot V^{\mathfrak{q}_{v}}$ is dense in $V$ if and only if $V=\mathfrak{q} \cdot v+V^{\mathfrak{q}_{v}}$.

The existence of a non-trivial generic stabiliser yields a Chevalley-type theorem for the field of invariants. Indeed, it follows from Proposition 1.4 that if $(Q: V)$ has a generic stabiliser, $v \in V$ is a generic point, and $U=V^{\mathfrak{q}_{v}}$, then

$$
\mathbb{k}(V)^{Q} \simeq \mathbb{k}(U)^{\mathrm{N}(U)}=\mathbb{k}(U)^{\mathrm{N}(U) / \mathrm{Z}(U)} .
$$

In this context, the group $W:=\mathrm{N}(U) / \mathrm{Z}(U)$ is called the Weyl group of the action $(Q: V)$. Notice that this $W$ is not necessarily finite.

The corresponding question for the algebras of invariants is much more subtle. The restriction homomorphism $\left.f \mapsto f\right|_{U}$ certainly induces an embedding $\mathbb{k}[V]^{Q} \hookrightarrow \mathbb{k}[U]^{\mathrm{N}(U) / \mathrm{Z}(U)}$. However, if $Q$ is non-reductive, then it is usually not onto.

\section{§2. Generic Stabilisers (centralisers) for the Adjoint Representation}

In what follows, $Q$ is a connected algebraic group. In this section, we elaborate on the existence of generic stabilisers and its consequences for the adjoint representations $\mathrm{Ad}: Q \rightarrow G L(\mathfrak{q})$ and ad $: \mathfrak{q} \rightarrow \mathfrak{g l}(\mathfrak{q})$.

For $x \in \mathfrak{q}$, the stabiliser $\mathfrak{q}_{x}$ is nothing but the centraliser of $x$ in $\mathfrak{q}$, so that we write $\mathfrak{z}_{\mathfrak{q}}(x)$ in place of $\mathfrak{q}_{x}$. The centraliser of $x$ in $Q$ is denoted by $Z_{Q}(x)$. If $(\mathfrak{q}$, ad $)$ has a generic stabiliser, then we also say that $\mathfrak{q}$ has a generic centraliser. By Lemma 1.6, a point $x \in \mathfrak{q}$ is generic if and only if

$$
[\mathfrak{q}, x]+\mathfrak{q}^{\mathfrak{z}^{\mathfrak{q}}}(x)=\mathfrak{q} .
$$

Since $\mathfrak{q}^{\mathfrak{z}_{\mathfrak{q}}}(x)$ is the centre of the Lie algebra $\mathfrak{z}_{\mathfrak{q}}(x)$ and $\operatorname{dim}[\mathfrak{q}, x]=\operatorname{dim} \mathfrak{q}-$ $\operatorname{dim} \mathfrak{z}_{\mathfrak{q}}(x)$, one immediately derives

2.1 Proposition. An algebraic Lie algebra $\mathfrak{q}$ has a generic centraliser if and only if there is an $x \in \mathfrak{q}$ such that

$$
\begin{gathered}
\mathfrak{z}_{\mathfrak{q}}(x) \text { is commutative and } \\
{[\mathfrak{q}, x] \oplus \mathfrak{z}_{\mathfrak{q}}(x)=\mathfrak{q} .}
\end{gathered}
$$

Equality (2.3) implies that $\operatorname{Im}(\operatorname{ad} x)=\operatorname{Im}(\operatorname{ad} x)^{2}$. The latter is never satisfied if $\operatorname{ad} x$ is nilpotent and $\operatorname{Im}(\operatorname{ad} x) \neq 0$. That is, if $\mathfrak{q}$ is nilpotent and $[\mathfrak{q}, \mathfrak{q}] \neq 0$, 
then $\mathfrak{q}$ has no generic centralisers. It also may happen that neither of the centralisers $\mathfrak{z}_{\mathfrak{q}}(x)$ is commutative. (Consider the Heisenberg Lie algebra $H_{n}$ of dimension $2 n+1$ for $n \geqslant 2$.) On the other hand, if there is a semisimple $x \in \mathfrak{q}$ such that $\mathfrak{z}_{\mathfrak{q}}(x)$ is commutative, then the conditions of Proposition 2.1 are satisfied, so that a generic centraliser exists. [Warning: this does not imply that the semisimple elements are dense in $\mathfrak{q}$.]

2.4 Lemma. Let $x \in \mathfrak{q}$ be a generic point. Then $\mathfrak{n}_{\mathfrak{q}}\left(\mathfrak{z}_{\mathfrak{q}}(x)\right)=\mathfrak{z}_{\mathfrak{q}}(x)$.

Proof. Assume that $\mathfrak{n}_{\mathfrak{q}}\left(\mathfrak{z}_{\mathfrak{q}}(x)\right) \neq \mathfrak{z}_{\mathfrak{q}}(x)$. In view of Eq. (2.3), there is then a nonzero $y \in \mathfrak{n}_{\mathfrak{q}}\left(\mathfrak{z}_{\mathfrak{q}}(x)\right) \cap[\mathfrak{q}, x]$. That is, $y=[s, x]$ for some $s \in \mathfrak{q}$. Then

$$
\left[y, \mathfrak{z}_{\mathfrak{q}}(x)\right]=\left[\left[s, \mathfrak{z}_{\mathfrak{q}}(x)\right], x\right] \subset[\mathfrak{q}, x]
$$

and hence $\left[y, \mathfrak{z}_{\mathfrak{q}}(x)\right]=0$. Thus, $y \in \mathfrak{z}_{\mathfrak{q}}(x) \cap[\mathfrak{q}, x]=0$, and we are done.

Recall that a subalgebra $\mathfrak{h}$ of $\mathfrak{q}$ is called a Cartan subalgebra if $\mathfrak{h}$ is nilpotent and $\mathfrak{n}_{\mathfrak{q}}(\mathfrak{h})=\mathfrak{h}$. Every Lie algebra has a Cartan subalgebra, and all Cartan subalgebras of $\mathfrak{q}$ are conjugate under $Q$, see [37, Ch. III].

2.5 Proposition. An algebraic Lie algebra $\mathfrak{q}$ has a generic centraliser if and only if the Cartan subalgebras of $\mathfrak{q}$ are commutative.

Proof. If $\mathfrak{q}$ has a generic centraliser, then, by Lemma 2.4, such a centraliser is a (commutative) Cartan subalgebra. Conversely, any Cartan subalgebra of $\mathfrak{q}$ is of the form $\mathfrak{h}=\left\{y \in \mathfrak{q} \mid(\operatorname{ad} x)^{n} y=0\right.$ for $\left.n \gg 0\right\}$ for some $x \in \mathfrak{q}$ [37, Ch. III.4, Cor. 2]. Therefore, the commutativity of $\mathfrak{h}$ implies that $\mathfrak{h}=\mathfrak{z}_{\mathfrak{q}}(x)$ and $\operatorname{ad} x$ is invertible on $[\mathfrak{q}, x]$.

As is already mentioned, the existence of a generic centraliser implies that of a generic isotropy group. For this reason, we always assume that a generic point $x$ has the property that $Z_{Q}(x)$ is a generic isotropy group. (This is only needed if a generic isotropy group is disconnected.)

2.6 Theorem. Suppose $\mathfrak{q}$ has a generic centraliser. Let $x \in \mathfrak{q}$ be a generic point such that $Z_{Q}(x)$ is a generic isotropy group. Then $(\mathrm{i}) \mathrm{Z}\left(\mathfrak{z}_{\mathfrak{q}}(x)\right)=$ $Z_{Q}(x)$ and (ii) $\mathbb{k}(\mathfrak{q})^{Q} \simeq \mathbb{k}\left(\mathfrak{z}_{\mathfrak{q}}(x)\right)^{W}$, where $W=N_{Q}\left(\mathfrak{z}_{\mathfrak{q}}(x)\right) / Z_{Q}(x)$ is a finite group.

Proof. (i) Since $x \in \mathfrak{z}_{\mathfrak{q}}(x)$, we have $\mathbf{Z}\left(\mathfrak{z}_{\mathfrak{q}}(x)\right) \subset Z_{Q}(x)$. Hence one has to prove that $Z_{Q}(x)$ acts trivially on $\mathfrak{z}_{\mathfrak{q}}(x)$. Assume that the fixed point space 
of $Z_{Q}(x)$ is a proper subspace of $\mathfrak{z}_{\mathfrak{q}}(x)$, say $M$. Since $\operatorname{dim} Q \cdot M \leqslant \operatorname{dim}[\mathfrak{q}, x]+$ $\operatorname{dim} M<\operatorname{dim} \mathfrak{q}, Q \cdot M$ cannot be dense in $\mathfrak{q}$, which contradicts the fact that $Z_{Q}(x)$ is a generic isotropy group.

(ii) This follows from Eq. (1.7) and Lemma 2.4.

Below, we state a property of generic points related to the dual space $\mathfrak{q}^{*}$.

2.7 Proposition. Let $x \in \mathfrak{q}$ be a generic point, as in Theorem 2.6. Then
(i) $\mathfrak{q}^{*}=x \cdot \mathfrak{q}^{*} \oplus\left(\mathfrak{q}^{*}\right)^{x}=x \cdot \mathfrak{q}^{*} \oplus\left(\mathfrak{q}^{*}\right)^{\mathfrak{z} \mathfrak{q}(x)}$ and
(ii) $\left(\mathfrak{q}^{*}\right)^{Z_{Q}(x)}=\left(\mathfrak{q}^{*}\right)^{\mathfrak{z} \mathfrak{q}(x)}$.

Proof. (i) We have $[\mathfrak{q}, x]^{\perp}=\left(\mathfrak{q}^{*}\right)^{x}$ and $\mathfrak{z}_{\mathfrak{q}}(x)^{\perp}=x \cdot \mathfrak{q}^{*}$. Hence the first equality follows from Eq. (2.3).

The second equality means that $\left(\mathfrak{q}^{*}\right)^{x}=\left(\mathfrak{q}^{*}\right)^{\mathfrak{z}_{\mathfrak{q}}(x)}$. Clearly, $\left(\mathfrak{q}^{*}\right)^{x} \supset\left(\mathfrak{q}^{*}\right)^{\mathfrak{z}_{\mathfrak{q}}(x)}$. Taking the annihilators provides the inclusion $[\mathfrak{q}, x] \subset\left[\mathfrak{q}, \mathfrak{z}_{\mathfrak{q}}(x)\right]$. Then using Eq. (2.2) and (2.3) yields

$$
\left[\mathfrak{q}, \mathfrak{z}_{\mathfrak{q}}(x)\right] \subset\left[\mathfrak{z}_{\mathfrak{q}}(x)+[\mathfrak{q}, x], \mathfrak{z}_{\mathfrak{q}}(x)\right]=\left[[\mathfrak{q}, x], \mathfrak{z}_{\mathfrak{q}}(x)\right]=\left[\left[\mathfrak{q}, \mathfrak{z}_{\mathfrak{q}}(x)\right], x\right] \subset[\mathfrak{q}, x] .
$$

(ii) In view of $(\mathbf{i}),\left(\mathfrak{q}^{*}\right)^{\mathfrak{z}_{\mathfrak{q}}(x)}$ is identified with $\left(\mathfrak{z}_{\mathfrak{q}}(x)\right)^{*}$. Hence the assertion stems from Theorem 2.6(i).

Thus, the very existence of a generic centraliser implies that $\mathfrak{q}$ has some properties in common with reductive Lie algebras. For instance, the Weyl group of $(Q: \mathfrak{q})$ is finite, and the decomposition of $\mathfrak{q}^{*}$ with respect to a generic element $x \in \mathfrak{q}$ is very similar to that of $\mathfrak{q}$. It will be shown below that there is a vast stock of such Lie algebras.

\section{$\S 3 . \quad$ Generic Stabilisers for the Coadjoint Representation}

In this section, we work with the coadjoint representations of $Q$ and $\mathfrak{q}$. Usually, we use lowercase Latin (resp. Greek) letters to denote elements of $\mathfrak{q}$ (resp. $\mathfrak{q}^{*}$ ). By Lemma 1.6, a point $\xi \in \mathfrak{q}^{*}$ is generic if and only if

$$
\mathfrak{q} \cdot \xi+\left(\mathfrak{q}^{*}\right)^{\mathfrak{q} \xi}=\mathfrak{q}^{*} .
$$

As was noticed by Tauvel and $\mathrm{Yu}$ [41], taking the annihilators yields a simple condition, entirely in terms of $\mathfrak{q}$. Namely, $\xi$ is generic if and only if

$$
\mathfrak{q}_{\xi} \cap\left[\mathfrak{q}, \mathfrak{q}_{\xi}\right]=\{0\}
$$

Below, we assume that $\left(\mathfrak{q}, \operatorname{ad}^{*}\right)$ has a generic stabiliser and thereby Eq. (3.1) is satisfied for some $\xi$. This readily implies that $\mathfrak{q}_{\xi}$ is commutative and $\mathfrak{n}_{\mathfrak{q}}\left(\mathfrak{q}_{\xi}\right)=$ 
$\mathfrak{z}_{\mathfrak{q}}\left(\mathfrak{q}_{\xi}\right)$. However, unlike the adjoint representation case, $\mathfrak{q}_{\xi}$ can be a proper subalgebra of $\mathfrak{z}_{\mathfrak{q}}\left(\mathfrak{q}_{\xi}\right)$. In other words, the Weyl group of $\left(Q: \mathfrak{q}^{*}\right)$ is not necessarily finite. Our goal is to understand what isomorphism (1.7) means in this situation. Set $\mathfrak{h}=\mathfrak{q}_{\xi}$ and $U=\left(\mathfrak{q}^{*}\right)^{\mathfrak{q}_{\xi}}$. Then we can write

$$
\mathbb{k}\left(\mathfrak{q}^{*}\right)^{Q} \simeq\left(\mathbb{k}(U)^{Z_{Q}(\mathfrak{h})^{o}}\right)^{N_{Q}(\mathfrak{h}) / Z_{Q}(\mathfrak{h})^{o}} .
$$

That is, one first takes the invariants of the connected group $Z_{Q}(\mathfrak{h})^{\circ}$, and then the invariants of the finite group $N_{Q}(\mathfrak{h}) / Z_{Q}(\mathfrak{h})^{o}$.

3.2 Lemma. $\operatorname{dim} U=\operatorname{dim} \mathfrak{z}_{\mathfrak{q}}(\mathfrak{h})$.

Proof. By Lemma 1.3 and Eq. (3.1), we have $\mathfrak{q} \cdot \xi \cap U=\mathfrak{z}_{\mathfrak{q}}(\mathfrak{h}) \cdot \xi$. Equating the dimensions of these spaces yields the assertion.

In view of this equality, it is tempting to interpret $U$ as the space of the coadjoint representation of $\mathfrak{z}_{\mathfrak{q}}(\mathfrak{h})=\operatorname{Lie} Z_{Q}(\mathfrak{h})^{o}$. However it seems to only be possible under an additional assumption on $\mathfrak{h}$.

3.3 Definition. We say that a subalgebra $\mathfrak{h}$ is near-toral if $[\mathfrak{q}, \mathfrak{h}] \cap \mathfrak{z}_{\mathfrak{q}}(\mathfrak{h})=$ $\{0\}$.

This condition is stronger than (3.1). It is obviously satisfied if $\mathfrak{h}$ is a toral Lie algebra (= Lie algebra of a torus).

Recall that the index of (a Lie algebra) $\mathfrak{q}$, ind $\mathfrak{q}$, is the minimal codimension of $Q$-orbits in $\mathfrak{q}^{*}$. Equivalently, ind $\mathfrak{q}=\operatorname{trdeg} \mathbb{k}\left(\mathfrak{q}^{*}\right)^{Q}$. If ind $\mathfrak{q}=0$, then $\mathfrak{q}$ is called Frobenius.

3.4 Theorem. Suppose the generic stabiliser $\mathfrak{h}$ is near-toral. Then

(i) $[\mathfrak{q}, \mathfrak{h}] \oplus \mathfrak{z}_{\mathfrak{q}}(\mathfrak{h})=\mathfrak{q}$ and $U \simeq \mathfrak{z}_{\mathfrak{q}}(\mathfrak{h})^{*}$;

(ii) ind $\mathfrak{q}=\operatorname{ind} \mathfrak{z}_{\mathfrak{q}}(\mathfrak{h})=\operatorname{dim} \mathfrak{h}$ and $\mathfrak{h}$ is the centre of $\mathfrak{z}_{\mathfrak{q}}(\mathfrak{h})$

Proof. (i) It is easily seen that $[\mathfrak{q}, \mathfrak{h}]^{\perp}=\left(\mathfrak{q}^{*}\right)^{\mathfrak{h}}=U$. Therefore Definition 3.3 says that $\mathfrak{z}_{\mathfrak{q}}(\mathfrak{h})^{\perp}+U=\mathfrak{q}^{*}$. From Lemma 3.2, it then follows that this sum (of $\mathfrak{z}_{\mathfrak{q}}(\mathfrak{h})$-modules) is direct. Hence $U \simeq \mathfrak{q}^{*} / \mathfrak{z}_{\mathfrak{q}}(\mathfrak{h})^{\perp} \simeq \mathfrak{z}_{\mathfrak{q}}(\mathfrak{h})^{*}$.

(ii) Since $\xi$ is generic and hence regular in $\mathfrak{q}^{*}$, we have ind $\mathfrak{q}=\operatorname{dim} \mathfrak{h}$. For $\nu \in U^{r e g}$, we have $U \cap \mathfrak{h}^{\perp}=U \cap \mathfrak{q} \cdot \nu=\mathfrak{z}_{\mathfrak{q}}(\mathfrak{h}) \cdot \nu$. In particular, $\operatorname{dim} \mathfrak{z}_{\mathfrak{q}}(\mathfrak{h}) \cdot \nu=$ $\operatorname{dim} U-\operatorname{dim} \mathfrak{h}$. Hence almost all $Z_{Q}(\mathfrak{h})$-orbits in $U$ are of codimension $\operatorname{dim} \mathfrak{h}$. This also means that the centre of $\mathfrak{z}_{\mathfrak{q}}(\mathfrak{h})$ cannot be larger than $\mathfrak{h}$.

3.5 Corollary. If the generic stabiliser $\mathfrak{h}$ is near-toral, then $\mathbb{k}\left(\mathfrak{q}^{*}\right)^{Q} \simeq$ $\left(\mathbb{k}\left(\mathfrak{z}_{\mathfrak{q}}(\mathfrak{h})^{*}\right)^{Z_{Q}(\mathfrak{h})^{o}}\right)^{F}$, where $F=N_{Q}(\mathfrak{h}) / Z_{Q}(\mathfrak{h})^{o}$ is finite. That is, one first takes 
the invariants of the coadjoint representation for a smaller Lie algebra and then the invariants of a finite group.

Under the assumption that $\mathfrak{h}$ is near-toral, $\mathfrak{s}:=\mathfrak{z}_{\mathfrak{q}}(\mathfrak{h})$ has the property that ind $\mathfrak{s}=\operatorname{dim} \mathfrak{z}(\mathfrak{s})$. The following results present some properties of such algebras.

3.6 Proposition. Suppose ind $\mathfrak{s}=\operatorname{dim} \mathfrak{z}(\mathfrak{s})$. Then

1. The closure of any regular $S$-orbit in $\mathfrak{s}^{*}$ is an affine space.

2. If $\mathfrak{z}(\mathfrak{s})$ is toral, then $\mathfrak{s} / \mathfrak{z}(\mathfrak{s})$ is Frobenius.

Proof. 1. If $y \in\left(\mathfrak{s}^{*}\right)^{\text {reg }}$, then $\mathfrak{s}_{y}=\mathfrak{z}(\mathfrak{s})$ and hence $\mathfrak{s} \cdot y=\mathfrak{z}(\mathfrak{s})^{\perp}$. Hence all points of the orbit $S \cdot y$ have one and the same tangent space. Therefore $S \cdot y$ is open and dense in the affine space $y+\mathfrak{z}(\mathfrak{s})^{\perp}$.

2. Since $\mathfrak{z}(\mathfrak{s})$ is reductive, one has a direct sum of Lie algebras $\mathfrak{s}=\mathfrak{r} \dot{+} \mathfrak{z}(\mathfrak{s})$, and ind $\mathfrak{r}=\operatorname{ind} \mathfrak{s}-\operatorname{ind} \mathfrak{z}(\mathfrak{s})=0$.

It is not, however, always true that $\mathfrak{s} / \mathfrak{z}(\mathfrak{s})$ is Frobenius. For instance, the Heisenberg Lie algebra $H_{n}$ has one-dimensional centre and ind $H_{n}=1$. But $H_{n} / \mathfrak{z}\left(H_{n}\right)$ is commutative, so that ind $\left(H_{n} / \mathfrak{z}\left(H_{n}\right)\right)=2 n$.

3.7 Examples. 1. Let $\mathfrak{b}$ be a Borel subalgebra of a simple Lie algebra $\mathfrak{g}$. Then $\left(\mathfrak{b}, \mathrm{ad}^{*}\right)$ has a generic stabiliser, which is always a toral Lie algebra, see e.g. [41]. If $\mathfrak{h}$ is such a stabiliser, then by Proposition $3.6, \mathfrak{z}_{\mathfrak{b}}(\mathfrak{h}) / \mathfrak{h}$ is a Frobenius Lie algebra. It is not hard to compute this quotient for all cases in which $\mathfrak{h} \neq 0$.

- If $\mathfrak{g}=\mathfrak{s l}_{n}$, then $\operatorname{dim} \mathfrak{h}=\left[\frac{n-1}{2}\right]$ and $\mathfrak{z}_{\mathfrak{b}}(\mathfrak{h}) / \mathfrak{h} \simeq \mathfrak{b}\left(\mathfrak{s l}_{2}\right)^{[n / 2]}$.

- If $\mathfrak{g}=\mathfrak{s o}_{4 n+2}$, then then $\operatorname{dim} \mathfrak{h}=1$ and $\mathfrak{z}_{\mathfrak{b}}(\mathfrak{h}) / \mathfrak{h} \simeq \mathfrak{b}\left(\mathfrak{s o}_{4 n}\right)$.

- If $\mathfrak{g}=\mathbf{E}_{6}$, then $\operatorname{dim} \mathfrak{h}=2$ and $\mathfrak{z}_{\mathfrak{b}}(\mathfrak{h}) / \mathfrak{h} \simeq \mathfrak{b}\left(\mathfrak{s o}_{8}\right)$.

2. If $\mathfrak{g}=\mathfrak{s l}_{n}$ or $\mathfrak{s p}_{2 n}$ and $\mathfrak{s}$ is a seaweed subalgebra of $\mathfrak{g}$, then a generic stabiliser for $\left(\mathfrak{s}, \mathrm{ad}^{*}\right)$ always exists, and it is a toral subalgebra [31]. For instance, let $\mathfrak{p} \subset \mathfrak{g l}_{2 n}$ be a maximal parabolic subalgebra whose Levi part is $\mathfrak{g l}_{n} \dot{+} \mathfrak{g l}_{n}$. Then a generic stabiliser for $\left(\mathfrak{p}, \mathrm{ad}^{*}\right)$ is $n$-dimensional and toral, and $\mathfrak{z}_{\mathfrak{p}}(\mathfrak{h}) / \mathfrak{h} \simeq \mathfrak{b}\left(\mathfrak{s l}_{2}\right)^{n}$.

3. There are non-trivial examples of Lie algebras such that a generic stabiliser for $\mathrm{ad}^{*}$ exists, is near-toral, and equals its own centraliser, but it is not toral. Let $e$ be a nilpotent element in $\mathfrak{g}=\mathfrak{s l}_{n}$ and $\mathfrak{q}=\mathfrak{z}_{\mathfrak{g}}(e)$. Then a generic stabiliser for the coadjoint representation of $\mathfrak{q}$ exists, see [48]. If $\mathfrak{h}$ is such a stabiliser, then the description of $\mathfrak{h}$ given in [48, Theorems $1 \& 5]$ shows that $\mathfrak{z}_{\mathfrak{q}}(\mathfrak{h})=\mathfrak{h}$. Hence, by Corollary $3.5, \mathbb{k}\left(\mathfrak{q}^{*}\right)^{Q}$ is the field of invariants of a finite group. 


\section{$\S 4$. Semi-Direct Products of Lie Algebras and Modules of Covariants}

In this section, we review some notions and results that will play the principal role in the following exposition.

(I) Recall a semi-direct product construction for Lie groups and algebras.

Let $V$ be a $Q$-module, and hence a $\mathfrak{q}$-module. Then $\mathfrak{q} \times V$ has a natural structure of Lie algebra, $V$ being an Abelian ideal in it. Explicitly, if $x, x^{\prime} \in \mathfrak{q}$ and $v, v^{\prime} \in V$, then

$$
\left[(x, v),\left(x^{\prime}, v^{\prime}\right)\right]=\left(\left[x, x^{\prime}\right], x \cdot v^{\prime}-x^{\prime} \cdot v\right) .
$$

This Lie algebra is denoted by $\mathfrak{q} \ltimes V$ or $\mathfrak{q} \oplus \epsilon V$. Accordingly, an element of this algebra is denoted by either $(x, v)$ or $x+\epsilon v$. Here $\epsilon$ is regarded as a formal symbol. Sometimes, e.g. if $V=\mathfrak{q}$, it is convenient to think of $\epsilon$ as element of the ring of dual numbers $\mathbb{k}[\epsilon]=\mathbb{k} \oplus \mathbb{k} \epsilon, \epsilon^{2}=0$. A connected algebraic group with Lie algebra $\mathfrak{q} \ltimes V$ is identified set-theoretically with $Q \times V$, and we write $Q \ltimes V$ for it. The product in $Q \ltimes V$ is given by

$$
(s, v)\left(s^{\prime}, v^{\prime}\right)=\left(s s^{\prime},\left(s^{\prime}\right)^{-1} \cdot v+v^{\prime}\right) .
$$

In particular, $(s, v)^{-1}=\left(s^{-1},-s \cdot v\right)$. The adjoint representation of $Q \ltimes V$ is given by the formula

$$
(\operatorname{Ad}(s, v))\left(x^{\prime}, v^{\prime}\right)=\left(\operatorname{Ad}(s) x^{\prime}, s \cdot v^{\prime}-x^{\prime} \cdot v\right),
$$

where $v, v^{\prime} \in V, x \in \mathfrak{q}$, and $s \in Q$.

Note that $V$ can be regarded as either a commutative unipotent subgroup of $Q \ltimes V$ or a commutative nilpotent subalgebra of $\mathfrak{q} \ltimes V$. Referring to $V$ as subgroup of $Q \ltimes V$, we write $1 \ltimes V$. A semi-direct product $\mathfrak{q} \ltimes V$ is said to be reductive if $\mathfrak{q}$ is a reductive (algebraic) Lie algebra.

(II) Our second important ingredient is the notion of modules of covariants.

Let $A$ be an algebraic group, acting on an affine variety $X$, and $V$ an $A$-module. The set of all $A$-equivariant morphisms from $X$ to $V$, denoted $\operatorname{Mor}_{A}(X, V)$, has a natural structure of $\mathbb{k}[X]^{A}$-module. This $\mathbb{k}[X]^{A}$-module is said to be the $\bmod$ ule of covariants (of type $V$ ). It is easily seen that $\operatorname{Mor}_{A}(X, V)$ can be identified with $(\mathbb{k}[X] \otimes V)^{A}$. For any $x \in X$, we denote by $\varepsilon_{x}$ the evaluation homomorphism $\operatorname{Mor}_{A}(X, V) \rightarrow V$, which takes $F$ to $F(x)$. Obviously, $\operatorname{Im}\left(\varepsilon_{x}\right) \subset V^{A_{x}}$.

Assume for a while that $A=G$ is reductive. Then the algebra $\mathbb{k}[X]^{G}$ is finitely generated and $\operatorname{Mor}_{G}(X, V)$ is a finitely generated $\mathbb{k}[X]^{G}$-module, see 
e.g. $[5,2.5],[46,3.12]$. A review of recent results on modules of covariants in the reductive case can be found in [42]. The following result is proved in [29, Theorem 1].

4.2 Theorem. If $\overline{G \cdot x}$ is normal and $\operatorname{codim} \overline{G \cdot x}(\overline{G \cdot x} \backslash G \cdot x) \geqslant 2$, then $\operatorname{Im}\left(\varepsilon_{x}\right)=V^{G_{x}}$.

Let $\mathfrak{g}^{\text {reg }}$ be the set of regular elements of $\mathfrak{g}$ and $T$ a maximal torus of $G$. The following fundamental result is due to Kostant [21, p. 385].

4.3 Theorem. Let $V$ be a $G$-module. Then $\operatorname{dim} V^{G_{x}}=\operatorname{dim} V^{T}$ for any $x \in \mathfrak{g}^{\text {reg }}$ and $\operatorname{Mor}_{G}(\mathfrak{g}, V)$ is a free $\mathbb{k}[\mathfrak{g}]^{G}$-module of rank $\operatorname{dim} V^{T}$.

In particular, if $V^{T}=0$, then there is no non-trivial $G$-equivariant mappings from $\mathfrak{g}$ to $V$. These modules of covariants are graded, and the degrees of minimal generating systems are uniquely determined. These degrees are called the generalised exponents of $V$. The multiset of generalised exponents of a $\mathfrak{g}$-module $V$ is denoted by $g$-exp $p_{\mathfrak{g}}(V)$. Similar results hold if $\mathfrak{g}$ is replaced with a "sufficiently good" $G$-module, see [47, Ch. III, §1] and [36, Prop. 4.3, 4.6]. Namely,

4.4 Theorem. Let $\tilde{V}$ be a $G$-module such that $\mathbb{k}[\tilde{V}]^{G}$ is a polynomial algebra and the quotient morphism $\pi: \tilde{V} \rightarrow \tilde{V} / / G$ is equidimensional. Then $\operatorname{Mor}_{G}(\tilde{V}, V)$ is a free $\mathbb{k}[\tilde{V}]^{G}$-module for any $G$-module $V$. Furthermore, if $(G$ : $\tilde{V})$ is stable, then the rank of $\operatorname{Mor}_{G}(\tilde{V}, V)$ equals $\operatorname{dim} V^{H}$, where $H$ is a generic isotropy group for $(G: \tilde{V})$.

An action $(G: V)$ is said to be stable, if the union of closed $G$-orbits is dense in $V$ (see [46, 7.5] and [44] about stable actions). If $(G: V)$ is stable, then a generic stabiliser is reductive and $\mathbb{k}(V)^{G}$ is the quotient field of $\mathbb{k}[V]^{G}$.

In some cases, a basis for free modules of covariants can explicitly be indicated. For any $f \in \mathbb{k}[V]$, the differential of $f$ can be regarded as a covector field on $V: v \mapsto d f_{v} \in V^{*}$. Starting with $f \in \mathbb{k}[V]^{G}$, one obtains in this way a covariant $d f \in \operatorname{Mor}_{G}\left(V, V^{*}\right)$. The following result of Thierry Vust appears in [47, Ch. III, § 2].

4.5 Theorem. Let a G-module $\tilde{V}$ satisfy all the assumptions of Theorem 4.4. Suppose also that $N_{G}(H) / H$ is finite. Let $f_{1}, \ldots, f_{m}$ be a set of basic invariants in $\mathbb{k}[\tilde{V}]^{G}$. Then $\operatorname{Mor}_{G}\left(\tilde{V}, \tilde{V}^{*}\right)$ is freely generated by $d f_{i}$, $i=1, \ldots, m$.

(III) Here we point out a connection between modules of covariants and invariants of semi-direct products. 
For $F \in \operatorname{Mor}_{A}(X, V)$, define the polynomial $\hat{F} \in \mathbb{k}\left[X \times V^{*}\right]^{A}$ by the rule $\widehat{F}(x, \xi)=\langle F(x), \xi\rangle$, where $\langle\rangle:, V \times V^{*} \rightarrow \mathbb{k}$ is the natural pairing.

4.6 Lemma. Consider the Lie algebra $\mathfrak{q} \ltimes V$ and the $\mathbb{k}[\mathfrak{q}]^{Q}$-module $\operatorname{Mor}_{Q}\left(\mathfrak{q}, V^{*}\right)$. Then for any $F \in \operatorname{Mor}_{Q}\left(\mathfrak{q}, V^{*}\right)$, we have $\widehat{F} \in \mathbb{k}[\mathfrak{q} \ltimes V]^{Q \ltimes V}$.

Proof. Clearly, $\widehat{F}$ is $Q$-invariant. The invariance with respect to $1 \ltimes V$ action means that

$$
\langle F(x), v\rangle=\left\langle F(x), v+x \cdot v^{\prime}\right\rangle
$$

holds for any $x \in \mathfrak{q}$ and $v, v^{\prime} \in V$. To this end, we notice that $\left\langle F(x), x \cdot v^{\prime}\right\rangle=$ $\left\langle x \cdot F(x), v^{\prime}\right\rangle$, and $x \cdot F(x)=0$, since $F: \mathfrak{q} \rightarrow V^{*}$ is a $Q$-equivariant morphism.

The point is that $\widehat{F}$ turns out to be invariant with respect to the action of the unipotent group $1 \ltimes V$.

\section{§5. Generic Stabilisers and Rational Invariants for Semi-Direct Products}

Given $Q$ and $V$, one may ask the following questions:

(Q1) When does a generic centraliser for $\mathfrak{q} \ltimes V$ exist? What are invarianttheoretic consequences of this?

It is easily seen that the existence of a generic centraliser for $\mathfrak{q}$ is a necessary condition. We will therefore assume that this is the case.

5.1 Theorem. Let $x \in \mathfrak{q}$ be a generic point. Suppose $V^{x}=V^{Z_{Q}(x)}$ and $V^{x} \oplus x \cdot V=V$. Then

(i) each point of the form $x+\epsilon v, v \in V^{\mathfrak{z}_{\mathfrak{q}}(x)}$, is generic and $\mathfrak{z}_{\mathfrak{q}}(x) \oplus \epsilon V^{\mathfrak{z}_{\mathfrak{q}}(x)}$ is a generic centraliser for $\mathfrak{q} \ltimes V$.

(ii) $\operatorname{trdeg} \mathbb{k}(\mathfrak{q} \ltimes V)^{Q \ltimes V}=\operatorname{trdeg} \mathbb{k}(\mathfrak{q})^{Q}+\operatorname{dim} V^{\mathfrak{z} \mathfrak{q}(x)}$;

(iii) The Weyl groups of $(\mathfrak{q}$, ad ) and $(\mathfrak{q} \ltimes V$, ad ) are isomorphic;

(iv) $\mathbb{k}(\mathfrak{q} \ltimes V)^{Q \ltimes V}$ is a purely transcendental extension of $\mathbb{k}(\mathfrak{q})^{Q}$.

Proof. Set $\mathfrak{h}=\mathfrak{z}_{\mathfrak{q}}(x), R=Q \ltimes V$, and $\mathfrak{r}=\mathfrak{q} \ltimes V$. It follows from the assumptions that $V^{x}=V^{\mathfrak{h}}$.

(i) Let $v \in V^{\mathfrak{h}}$ be arbitrary. Let us verify that Proposition 2.1 applies here. A 
direct calculation shows that $\mathfrak{z} \mathfrak{r}(x+\epsilon v)=\mathfrak{h} \oplus \epsilon V^{\mathfrak{h}}$ and this algebra is commutative. Next,

$$
[\mathfrak{r}, x+\epsilon v]=\{[z, x]+\epsilon(z \cdot v) \mid z \in \mathfrak{q}\}+\epsilon(x \cdot V) .
$$

Notice that $\mathfrak{q} \cdot v=([\mathfrak{q}, x] \oplus \mathfrak{h}) \cdot v=[\mathfrak{q}, x] \cdot v=x \cdot(\mathfrak{q} \cdot v) \subset x \cdot V$. Hence $z \cdot v \subset$ $x \cdot V$ for any $z \in \mathfrak{q}$ and $[\mathfrak{r}, x+\epsilon v]=[\mathfrak{q}, x] \oplus \epsilon(x \cdot V)$. Therefore the equality $[\mathfrak{r}, x+\epsilon v] \oplus \mathfrak{z}_{\mathfrak{r}}(x+\epsilon v)=\mathfrak{r}$ is equivalent to that $V^{x} \oplus x \cdot V=V$.

(ii) By part (i), $\tilde{\mathfrak{h}}:=\mathfrak{h} \oplus \epsilon V^{\mathfrak{h}}$ is a generic centraliser for $\mathfrak{r}$. Since $\operatorname{trdeg} \mathbb{k}(\mathfrak{q})^{Q}=\operatorname{dim} \mathfrak{h}$, the claim follows.

(iii) Using formula (4.1), one easily verifies that $N_{R}(\tilde{\mathfrak{h}})=N_{Q}(\mathfrak{h}) \ltimes V^{\mathfrak{h}}$ and $Z_{R}(\tilde{\mathfrak{h}})=Z_{Q}(\mathfrak{h}) \ltimes V^{\mathfrak{h}}$. Hence using Theorem 2.6, we obtain

$$
\tilde{W}=N_{R}(\tilde{\mathfrak{h}}) / Z_{R}(\tilde{\mathfrak{h}}) \simeq N_{Q}(\mathfrak{h}) / Z_{Q}(\mathfrak{h})=W .
$$

(iv) Here we may work entirely with invariants of $W$. In view of (iii) and Theorem 2.6, it suffices to prove that $\mathbb{k}(\mathfrak{h})^{W} \hookrightarrow \mathbb{k}\left(\mathfrak{h} \oplus V^{\mathfrak{h}}\right)^{W}$ is a purely transcendental extension. Actually, a transcendence basis of $\mathbb{k}\left(\mathfrak{h} \oplus V^{\mathfrak{h}}\right)^{W}$ over $\mathbb{k}(\mathfrak{h})^{W}$ can explicitly be constructed. This follows from Theorem 5.2 below, since the representation of $W$ on $\mathfrak{h}$ is faithful.

The following result concerns fields of invariants of reductive algebraic groups.

Recall from $\S 4(\mathrm{III})$ that one may associate the invariant $\widehat{F} \in \mathbb{k}\left[V_{1} \times V_{2}\right]^{G}$ to any $F \in \operatorname{Mor}_{G}\left(V_{1}, V_{2}^{*}\right)$. If $D$ is a domain, then we write $D_{(0)}$ for the field of fractions.

5.2 Theorem. Let $\rho_{i}: G \rightarrow G L\left(V_{i}\right), i=1,2$, be representations of a reductive group $G$. Set $m=\operatorname{dim} V_{2}$ and $J=\mathbb{k}\left[V_{1}\right]^{G}$. Suppose that a generic isotropy subgroup for $\left(G: V_{1}\right)$ is trivial, and $\left(G: V_{1}\right)$ is stable. Then

(i) $\operatorname{dim}_{J_{(0)}} \operatorname{Mor}_{G}\left(V_{1}, V_{2}^{*}\right) \otimes_{J} J_{(0)}=m$;

(ii) Let $F_{1}, \ldots, F_{m} \in \operatorname{Mor}_{G}\left(V_{1}, V_{2}^{*}\right)$ be covariants such that $\left\{F_{i} \otimes 1 \mid i=\right.$ $1, \ldots, m\}$ form a basis for the $J_{(0)}$-vector space in (i). Then $\mathbb{k}\left(V_{1} \oplus V_{2}\right)^{G}=$ $\mathbb{k}\left(V_{1}\right)^{G}\left(\widehat{F}_{1}, \ldots, \widehat{F}_{m}\right)$. In other words, any such basis for $\operatorname{Mor}_{G}\left(V_{1}, V_{2}^{*}\right) \otimes_{J}$ $J_{(0)}$ gives rise to a transcendence basis for the field $\mathbb{k}\left(V_{1} \oplus V_{2}\right)^{G}$ over $\mathbb{k}\left(V_{1}\right)^{G}$.

Proof. (i) Because $\left(G: V_{1}\right)$ is stable, $J_{(0)}=\mathbb{k}\left(V_{1}\right)^{G}$. Since $\operatorname{Mor}_{G}\left(V_{1}, V_{2}^{*}\right)$ is a finitely-generated $J$-module, $\underline{M}=\operatorname{Mor}_{G}\left(V_{1}, V_{2}^{*}\right) \otimes_{J} J_{(0)}$ is a finitedimensional $J_{(0)}$-vector space. By the assumptions, there is an $x \in V_{1}$ such 
that the isotropy group $G_{x}$ is trivial and $G \cdot x=\overline{G \cdot x}$. Then by Theorem 4.2,

$(\diamond) \quad$ the evaluation map $\varepsilon_{x}: \operatorname{Mor}_{G}\left(V_{1}, V_{2}^{*}\right) \rightarrow V_{2}^{*}=\left(V_{2}^{*}\right)^{G_{x}}$ is onto.

Hence $\operatorname{dim} \underline{M} \geqslant m$. On the other hand, it cannot be greater than $m$.

(ii) In view of Theorem 1.1, it suffices to prove that $\mathbb{k}\left(V_{1}\right)^{G}\left(\widehat{F}_{1}, \ldots, \widehat{F}_{m}\right)$ separates the generic $G$-orbits in $V_{1} \oplus V_{2}$. First, the field $\mathbb{k}\left(V_{1}\right)^{G}$ separates the generic $G$-orbits in $V_{1}$. Therefore, for generic points $\left(x_{1}, x_{2}\right), x_{i} \in V_{i}$, the first coordinate is determined uniquely up to $G$-conjugation by the values $f\left(x_{1}\right)$, where $f$ runs over $\mathbb{k}\left(V_{1}\right)^{G}$. By condition $(\diamond), F_{1}\left(x_{1}\right), \ldots, F_{m}\left(x_{1}\right)$ form a basis for $V_{2}^{*}$ if $x_{1}$ is generic. Hence given a generic $x_{1}$ and arbitrary values of the invariants $\widehat{F}_{i}$, the second coordinate (i.e., $x_{2}$ ) is uniquely determined.

Remarks. 1. Most of the assumptions of Theorem 5.2 are always satisfied if $G$ is either finite or semisimple. For $G$ finite, it suffices to only require that $\rho_{1}$ is faithful. For $G$ semisimple, it suffices to require that a generic isotropy group of $\left(G: V_{1}\right)$ is trivial.

2. The assertion that the field extension in (ii) is purely transcendental is known, see e.g. $[12$, p. 6$]$. But the explicit construction of a transcendence basis via modules of covariants seems to be new.

The following assertion demonstrates important instances, where Theorem 5.1 applies.

5.3 Proposition. Theorem 5.1 applies to the following $\mathfrak{q}$-modules $V$ :

1. $\mathfrak{q}$ is an arbitrary Lie algebra having a generic centraliser and $V$ is either $\mathfrak{q}$ or $\mathfrak{q}^{*}$.

2. $\mathfrak{q}=\mathfrak{g}$ is reductive and $V$ is an arbitrary $\mathfrak{g}$-module.

Proof. 1. For $\mathfrak{q} \ltimes \mathfrak{q}$, the conditions of Theorem 5.1 are satisfied in view of Proposition 2.1 and Theorem 2.6. For $\mathfrak{q} \ltimes \mathfrak{q}^{*}$, these conditions are satisfied in view of Proposition 2.7.

2. Here $x \in \mathfrak{g}$ is a regular semisimple element and $Z_{G}(x)$ is a maximal torus. Therefore $V^{x}$ is the zero weight space of $V$ and $x \cdot V$ is the sum of all other weight spaces.

Remark. For the semi-direct products as in Proposition 5.3(2), we are able to describe the polynomial invariants, see $\S 6$.

A Lie algebra is said to be quadratic whenever its adjoint and coadjoint representations are equivalent. It is easily seen that $\mathfrak{q} \ltimes \mathfrak{q}^{*}$ is quadratic for any Lie algebra $\mathfrak{q}$. For, if $\langle$,$\rangle is the pairing of \mathfrak{q}$ and $\mathfrak{q}^{*}$, then the formula $\left(x_{1}+\epsilon \xi_{1}, x_{2}+\epsilon \xi_{2}\right)=\left\langle x_{1}, \xi_{2}\right\rangle+\left\langle x_{2}, \xi_{1}\right\rangle$ determines a non-degenerate symmetric 
$\mathfrak{q} \ltimes \mathfrak{q}^{*}$-invariant form. For $\mathfrak{q} \ltimes \mathfrak{q}^{*}$, there is no difference between the adjoint and coadjoint representations. So, previous results of this section describe some properties of the coadjoint representation of $\mathfrak{q} \ltimes \mathfrak{q}^{*}$ as well. However, for an arbitrary $V$ the adjoint and coadjoint representation of $\mathfrak{q} \ltimes V$ are very different. Hence our second problem is:

(Q2) When does a generic stabiliser for $\left(\mathfrak{q} \ltimes V\right.$, ad $\left.\mathrm{d}^{*}\right)$ exist? What are invarianttheoretic consequences of this?

This problem is quite different from (Q1). It seems to be more involved and restrictive.

Set $\mathfrak{r}=\mathfrak{q} \ltimes V$ and $R=Q \ltimes V$. The dual space $\mathfrak{r}^{*}$ is identified with $\mathfrak{q}^{*} \oplus V^{*}$, and a typical element of it is denoted by $\eta=(\alpha, \xi)$. For $(s, v) \in \mathfrak{r}$, the coadjoint representation is given by

$$
\left(\operatorname{ad}_{\mathfrak{r}}^{*}(s, v)\right)(\alpha, \xi)=\left(\operatorname{ad}_{\mathfrak{q}}^{*}(s)(\alpha)-v * \xi, s \cdot \xi\right) .
$$

Here the mapping $\left((s, \xi) \in \mathfrak{q} \times V^{*}\right) \mapsto\left(s \cdot \xi \in V^{*}\right)$ is the natural $\mathfrak{q}$-module structure on $V^{*}$, and $\left((v, \xi) \in V \times V^{*}\right) \mapsto\left(v * \xi \in \mathfrak{q}^{*}\right)$ is the moment mapping with respect to the symplectic structure on $V \times V^{*}$.

To describe the stabiliser of any point in $\mathfrak{r}^{*}$, we need some notation. For $\alpha \in \mathfrak{q}^{*}$, let $\mathcal{K}_{\alpha}$ denote the Kirillov form on $\mathfrak{q}$ associated with $\alpha$, i.e., $\mathcal{K}_{\alpha}\left(s_{1}, s_{2}\right)=$ $\left\langle\alpha,\left[s_{1}, s_{2}\right]\right\rangle$. Then $\operatorname{ker}\left(\mathcal{K}_{\alpha}\right)=\mathfrak{q}_{\alpha}$, the stabiliser of $\alpha$. If $\mathfrak{h}$ is a subalgebra of $\mathfrak{q}$, then $\left.\mathcal{K}_{\alpha}\right|_{\mathfrak{h}}$ can also be regarded as the Kirillov form associated with $\left.\alpha\right|_{\mathfrak{h}} \in \mathfrak{h}^{*}$.

5.5 Proposition. For any $\eta=(\alpha, \xi) \in \mathfrak{r}^{*}$, we have

$$
\mathfrak{r}_{\eta}=\left\{(s, v) \mid s \in \operatorname{ker}\left(\left.\mathcal{K}_{\alpha}\right|_{\mathfrak{q}_{\xi}}\right) \quad \& \quad \operatorname{ad}_{\mathfrak{q}}^{*}(s) \alpha=v * \xi\right\} .
$$

Proof. Straightforward. The first condition imposed on $s$ guarantees us the equality $s \cdot \xi=0$ and that the equation $\operatorname{ad}_{\mathfrak{q}}^{*}(s) \alpha=v * \xi$ has a solution $v$ for any such $s$.

It follows that $\mathfrak{r}_{\eta}$ is a direct sum of the space $\{w \in V \mid w * \xi=0\}=(\mathfrak{q} \cdot \xi)^{\perp}$, sitting in $V$, and a space of dimension $\operatorname{dim} \operatorname{ker}\left(\left.\mathcal{K}_{\alpha}\right|_{\mathfrak{q}_{\xi}}\right)$, which is embedded in $\mathfrak{q} \ltimes V$ somehow diagonally. (We will see below that under additional constraints this second space lies entirely in $\mathfrak{q}$.)

A result of Raïs on semi-direct products [32] describes $\mathfrak{r}$-regular points in $\mathfrak{r}^{*}$ and gives the value of ind $\mathfrak{r}$, that is, the dimension of the stabilizer of the $\mathfrak{r}$-regular points in $\mathfrak{r}^{*}$. Namely, if $\xi \in V^{*}$ is $\mathfrak{q}$-regular, then $(\alpha, \xi)$ is $\mathfrak{r}$-regular 
if and only if $\alpha$ is $\mathfrak{q}_{\xi}$-regular as element of $\mathfrak{q}_{\xi}^{*}$ (with respect to the coadjoint representation of $\mathfrak{q}_{\xi}$ ). By a theorem of Duflo-Vergne [13], the stabiliser of any regular point in the coadjoint representation is commutative, see also [30, 1.8] for an invariant-theoretic proof.

It seems to be difficult to find out a general condition ensuring that Eq. (3.1) holds for some regular point in $\mathfrak{r}^{*}$. For this reason, we only look at the three cases occurring already in Proposition 5.3 in connection with generic centralisers.

- If $\left(\mathfrak{q}, \mathrm{ad}^{*}\right)$ has a generic stabiliser, then $\left(\mathfrak{q} \ltimes \mathfrak{q}, \mathrm{ad}^{*}\right)$ has.

Indeed, if $\mathfrak{q}_{\xi}$ is a generic stabiliser $\left(\xi \in \mathfrak{q}^{*}\right)$, then $\mathfrak{q}_{\xi} \ltimes \mathfrak{q}_{\xi}$ is the stabiliser of $\eta=(0, \xi) \in(\mathfrak{q} \ltimes \mathfrak{q})^{*}$ and $\left[\mathfrak{q} \ltimes \mathfrak{q}, \mathfrak{q}_{\xi} \ltimes \mathfrak{q}_{\xi}\right] \cap\left(\mathfrak{q}_{\xi} \ltimes \mathfrak{q}_{\xi}\right)=\{0\}$.

- If $\left(\mathfrak{q}, \operatorname{ad}^{*}\right)$ has a generic stabiliser, then $\left(\mathfrak{q} \ltimes \mathfrak{q}^{*}, \operatorname{ad}^{*}\right)$ may have no generic stabilisers.

Example. Let $\mathfrak{q}$ be the 3 -dimensional Heisenberg algebra $H_{1}$. The generic stabiliser for $\left(\mathfrak{q}, \mathrm{ad}^{*}\right)$ exists and equals the centre of $\mathfrak{q}$. But $\hat{\mathfrak{q}}=\mathfrak{q} \ltimes \mathfrak{q}^{*}$ is nilpotent and quadratic. Therefore $\left(\hat{\mathfrak{q}}, \mathrm{ad}^{*}\right) \simeq(\hat{\mathfrak{q}}$, ad $)$ has no generic stabiliser.

- Suppose $\mathfrak{q}=\mathfrak{g}$ is reductive. Then $\mathfrak{g} \simeq \mathfrak{g}^{*}$.

By [34], $\left(\mathfrak{g}: V^{*}\right)$ always has a generic stabiliser. Assume that this stabiliser is reductive. There is no much harm in it, since there are finitely many $\mathfrak{g}$-modules whose generic stabiliser is not reductive. Then our goal is to prove that the existence of a generic stabiliser for $\left(\mathfrak{r}, \mathrm{ad}^{*}\right)$ imposes a very strong constraint on the action $(G: V)$.

Let $\Omega_{V^{*}}$ be the open subset of $\mathfrak{g}$-generic points in $V^{*}$. Fix a generic stabiliser $\mathfrak{h} \subset \mathfrak{g}$ and a Cartan subalgebra $\mathfrak{t}_{\mathfrak{h}} \subset \mathfrak{h}$.

5.6 Lemma. There is an open $R$-stable subset $\Xi \subset\left(\mathfrak{r}^{*}\right)^{r e g} \cap\left(\mathfrak{g} \times \Omega_{V^{*}}\right)$ such that if $\eta=(\alpha, \xi) \in \Xi$, then $\mathfrak{r}_{\eta}$ is a direct sum of two spaces, one lying in $\mathfrak{g}^{*}$ and another lying in $V^{*}$. Furthermore, eventually replacing $\eta$ with an $H$-conjugate point, one can achieve that $\mathfrak{r}_{\eta}=\mathfrak{t}_{\mathfrak{h}} \ltimes(\mathfrak{g} \cdot \xi)^{\perp}$.

Proof. Since $\mathfrak{h}$ is reductive, the $\mathfrak{h}$-modules $\mathfrak{h}$ and $\mathfrak{h}^{*}$ can be identified using the restriction to $\mathfrak{h}$ of a non-degenerate $\mathfrak{g}$-invariant symmetric bilinear form on $\mathfrak{g}$. Suppose $\eta=(\alpha, \xi) \in\left(\mathfrak{r}^{*}\right)^{r e g} \cap\left(\mathfrak{g} \times \Omega_{V^{*}}\right)$. Without loss of generality, assume that $\mathfrak{g}_{\xi}=\mathfrak{h}$. As was explained above, the $\mathfrak{r}$-regularity of $\eta$ means that $\alpha$ is $\mathfrak{h}$-regular as an element of $\mathfrak{h}^{*}$. Having identified $\mathfrak{h}^{*}$ and $\mathfrak{h}$, we may assume that $\alpha$ is regular semisimple. This last condition distinguishes the required subset $\Xi$. Then $\operatorname{ker}\left(\left.\mathcal{K}_{\alpha}\right|_{\mathfrak{h}}\right)$ is a Cartan subalgebra of $\mathfrak{h}$, and if $s \in \operatorname{ker}\left(\left.\mathcal{K}_{\alpha}\right|_{\mathfrak{h}}\right)$, then $\operatorname{ad}_{\mathfrak{g}}^{*}(s) \alpha=0$. Comparing this with Proposition 5.5, we see that $\mathfrak{r}_{\eta}=$ 
$\operatorname{ker}\left(\left.\mathcal{K}_{\alpha}\right|_{\mathfrak{h}}\right) \ltimes(\mathfrak{g} \cdot \xi)^{\perp}$. Taking an $H$-conjugate, which does not affect $\xi$, we may achieve that $\operatorname{ker}\left(\left.\mathcal{K}_{\alpha}\right|_{\mathfrak{h}}\right)=\mathfrak{t}_{\mathfrak{h}}$.

Thus, for almost all $\mathfrak{r}$-regular points in $\mathfrak{r}^{*}$, their stabilisers are conjugate to subalgebras of the form $\tilde{\mathfrak{h}}=\mathfrak{t}_{\mathfrak{h}} \ltimes(\mathfrak{g} \cdot \xi)^{\perp}$. Set $U=(\mathfrak{g} \cdot \xi)^{\perp}$. By the very construction, $U$ is $\mathfrak{h}$-stable. Since $\eta$ is regular and therefore $\mathfrak{r}_{\eta}$ is commutative, $\mathfrak{t}_{\mathfrak{h}}$ acts trivially on $U$, i.e., $\mathfrak{t}_{\mathfrak{h}} \cdot U=0$.

\subsection{Proposition.}

1. Suppose $\left(\mathfrak{r}, \operatorname{ad}^{*}\right)$ has a generic stabiliser. Then $\mathfrak{g} \cdot U \cap U=\{0\}$.

2. If $\mathfrak{h}=0$, then the converse is also true.

Proof. 1. By Lemma 5.6 and Eq. (3.1), $\left(\mathfrak{r}, \mathrm{ad}^{*}\right)$ has a generic stabiliser if and only if $[\mathfrak{r}, \tilde{\mathfrak{h}}] \cap \tilde{\mathfrak{h}}=\{0\}$. We have

$$
[\mathfrak{r}, \tilde{\mathfrak{h}}]=\left[\mathfrak{g} \ltimes V, \mathfrak{t}_{\mathfrak{h}} \ltimes U\right]=\left[\mathfrak{g}, \mathfrak{t}_{\mathfrak{h}} \ltimes U\right]+\mathfrak{t}_{\mathfrak{h}} \cdot V .
$$

Clearly, $\mathfrak{g} \cdot U$ is a subspace of $\left[\mathfrak{g}, \mathfrak{t}_{\mathfrak{h}} \ltimes U\right]$. Hence we get the condition that $\mathfrak{g} \cdot U \cap U=\{0\}$.

2. Let $V=U \oplus V^{\prime}$ be an $\mathfrak{h}$-stable decomposition. Then $\mathfrak{t}_{\mathfrak{h}} \cdot V=\mathfrak{t}_{\mathfrak{h}} \cdot V^{\prime} \subset V^{\prime}$. Hence this summand causes no harm. If $\mathfrak{h}=0$, then $\left[\mathfrak{g}, \mathfrak{t}_{\mathfrak{h}} \ltimes U\right]=\mathfrak{g} \cdot U$. Therefore the condition $\mathfrak{g} \cdot U \cap U=\{0\}$ appears to be necessary and sufficient for the existence of a generic stabiliser.

Recall from [11] the notion of a polar representation of a reductive group. Let $v \in V$ be semisimple, i.e., $G \cdot v$ is closed. Define $\mathfrak{c}_{v}=\{x \in V \mid \mathfrak{g} \cdot x \subset \mathfrak{g} \cdot v\}$. Then $(G: V)$ is said to be polar if there is a semisimple $v \in V$ such that $\operatorname{dim} \mathfrak{c}_{v}=\operatorname{dim} V / / G$. Such $\mathfrak{c}$ is called a Cartan subspace. Polar representations have a number of nice (and hence restrictive) properties. For instance, all points of $\mathfrak{c}$ are semisimple, all Cartan subspaces are $G$-conjugate, the group $W_{\mathfrak{c}}:=\mathrm{N}(\mathfrak{c}) / Z(\mathfrak{c})$ is finite, and $\mathbb{k}[V]^{G} \simeq \mathbb{k}[\mathfrak{c}]^{W_{\mathfrak{c}}}[11]$. The latter implies that $\mathbb{k}[V]^{G}$ is polynomial and the morphism $\pi_{G}: V \rightarrow V / / G$ is equidimensional [26].

Our main result related to Question (Q2) is:

5.8 Theorem. Suppose the action $(G: V)$ is stable. Then $\left(\mathfrak{r}=\mathfrak{g} \ltimes V, \operatorname{ad}^{*}\right)$ has a generic stabiliser if and only if $(G: V)$ is a polar representation.

Proof. 1. Suppose $\left(\mathfrak{g} \ltimes V\right.$, $\left.\mathrm{ad}^{*}\right)$ has a generic stabiliser.

Choose $\eta=(\alpha, \xi) \in \Xi$ as prescribed by Lemma 5.6 , so that $\mathfrak{r}_{\eta}=\mathfrak{t}_{\mathfrak{h}} \ltimes U$ is a generic stabiliser and hence $\mathfrak{g} \cdot U \cap U=\{0\}$ (Proposition 5.7). In view of 
stability, we may also assume that $\xi$ is (g-regular and) semisimple. Let us prove that $U$ is a Cartan subspace of $V$.

As is well known, $\operatorname{dim} V / / G=\operatorname{dim} V^{*} / / G$ and $(G: V)$ is stable if and only if $\left(G: V^{*}\right)$ is, see e.g. [44]. By the stability hypothesis,

$$
\max _{\nu \in V^{*}} \operatorname{dim} G \cdot \nu=\max _{v \in V} \operatorname{dim} G \cdot v=\operatorname{dim} V-\operatorname{dim} V / / G .
$$

Hence $G \cdot \xi=\operatorname{dim} V-\operatorname{dim} V / / G$ and $\operatorname{dim} U=\operatorname{dim} V / / G$.

Claim. There is a closed $G$-orbit of maximal dimension meeting $U$.

Proof of the claim. The proof of main results in [11] is based on transcendental methods (compact real forms of $G$, Kempf-Ness theory). This is an excuse for our using similar methods below. In the next paragraph, we assume that $\mathbb{k}=\mathbb{C}$.

Let $G_{c}$ be a maximal compact subgroup of $G$ with Lie algebra $\mathfrak{g}_{c}$. Fix a $G_{c}$-invariant Hermitian form $\langle$,$\rangle on V^{*}$. Without loss of generality, we may assume that $\xi$ is of minimal length in $G \cdot \xi$ and hence $\langle\mathfrak{g} \cdot \xi, \xi\rangle=0$, see [11, Sect. 1]. Upon the identification the $\mathfrak{g}_{c}$-modules $V$ and $V^{*}$ via $\langle\rangle,, \xi$ appears to be a point of $U$. If $\tilde{v} \in U$ corresponds to $\xi$ under this identification, then we still have $\left\langle\mathfrak{g}_{c} \cdot \tilde{v}, \tilde{v}\right\rangle=0$, and therefore $\langle\mathfrak{g} \cdot \tilde{v}, \tilde{v}\rangle=0$. Hence $G \cdot \tilde{v}$ is closed [11, Theorem 1.1]. Since $\left(\mathfrak{g}_{c}\right)_{\xi}=\left(\mathfrak{g}_{c}\right)_{\tilde{v}}$ and $\left(\mathfrak{g}_{c}\right)_{\tilde{v}}$ is a compact real form of $\mathfrak{g}_{\tilde{v}}$ [11, Prop. 1.3], we conclude that $\operatorname{dim} \mathfrak{g}_{\tilde{v}}=\operatorname{dim} \mathfrak{g}_{\xi}=\operatorname{dim} \mathfrak{h}$.

For $\tilde{v} \in U$, we have $\operatorname{dim} \mathfrak{g} \cdot \tilde{v}=\operatorname{dim} V-\operatorname{dim} U$. Hence $\mathfrak{g} \cdot \tilde{v}=\mathfrak{g} \cdot U$ for dimension reason. In particular, $\mathfrak{g} \cdot y \subset \mathfrak{g} \cdot \tilde{v}$ for any $y \in U$. Thus, $U$ satisfies all conditions in the definition of a Cartan subspace.

2. Suppose $(G: V)$ is stable and polar.

Let $v \in V$ be a regular semisimple element and $\mathfrak{c}=\mathfrak{c}_{v}$ the corresponding Cartan subspace. Then $V=\mathfrak{g} \cdot \mathfrak{c} \oplus \mathfrak{c}$ and $\mathfrak{g} \cdot \mathfrak{c}=\mathfrak{g} \cdot v\left[11\right.$, Section 2]. Set $\mathfrak{h}=\mathfrak{g}_{v}$. The Lie algebra $\mathfrak{s}:=\mathfrak{t}_{\mathfrak{h}} \ltimes \mathfrak{c}$ is commutative, and a direct verification shows that it satisfies Eq. (3.1). Indeed,

$$
\left[\mathfrak{g} \ltimes V, \mathfrak{t}_{\mathfrak{h}} \ltimes \mathfrak{c}\right]=\left[\mathfrak{g}, \mathfrak{t}_{\mathfrak{h}} \ltimes \mathfrak{c}\right]+\mathfrak{t}_{\mathfrak{h}} \cdot V .
$$

Using the $\mathfrak{t}_{\mathfrak{h}}$-stable decomposition $V=\mathfrak{g} \cdot \mathfrak{c} \oplus \mathfrak{c}$, we see that $\mathfrak{t}_{\mathfrak{h}} \cdot V \subset \mathfrak{g} \cdot \mathfrak{c}$. As for the first summand, its $\mathfrak{g}$-component does not belong to $\mathfrak{t}_{\mathfrak{h}}$ and its $V$-component belongs to $\mathfrak{g} \cdot \mathfrak{c}$. Hence $\left[\mathfrak{g} \ltimes V, \mathfrak{t}_{\mathfrak{h}} \ltimes \mathfrak{c}\right] \cap\left(\mathfrak{t}_{\mathfrak{h}} \ltimes \mathfrak{c}\right)=\{0\}$. It remains to find an $\eta \in \mathfrak{r}^{*}$ such that $\mathfrak{r}_{\eta}=\mathfrak{s}$.

The dual version of the previous Claim shows that $(\mathfrak{g} \cdot \mathfrak{c})^{\perp}$ is a Cartan subspace of $V^{*}$ and that, for sufficiently general $\xi \in(\mathfrak{g} \cdot \mathfrak{c})^{\perp}$, we have $\mathfrak{g}_{\xi}=\mathfrak{h}$ 
and $\mathfrak{g} \cdot \xi=\mathfrak{c}^{\perp}$. Now, take an $\alpha \in \mathfrak{g}^{*}$ such that under the identification $\mathfrak{g}^{*} \simeq \mathfrak{g}$ it becomes a regular element of $\mathfrak{t}_{\mathfrak{h}}\left(\right.$ i.e., $\left.\alpha \in\left(\mathfrak{t}_{\mathfrak{h}}\right)^{\text {reg }}\right)$. Then $\gamma=(\alpha, \xi) \in\left(\mathfrak{r}^{*}\right)^{\text {reg }}$ and $\mathfrak{r}_{\gamma}=\mathfrak{s}$.

We mention without proof the following consequence of Theorem 5.8.

5.9 Corollary. If a generic stabiliser $\mathfrak{h}$ for $\left(\mathfrak{r}, \operatorname{ad}^{*}\right)$ is near-toral, then $\operatorname{rk} \mathfrak{h}=\operatorname{rk} \mathfrak{g}$ and $U=V^{\mathfrak{h}}$. In case of $\mathfrak{g}$ simple, this implies that $V$ is either the adjoint or "little adjoint" $\mathfrak{g}$-module. (The latter means that the highest weight is the short dominant root, in case $\mathfrak{g}$ has roots of different length.)

Remark. It may happen that a generic stabiliser for $\left(G: V^{*}\right)$ is not reductive, but $\left(\mathfrak{g} \ltimes V, \mathrm{ad}^{*}\right)$ still has a generic stabiliser. Indeed, there are $G$-modules $V$ such that $\mathfrak{r}=\mathfrak{g} \ltimes V$ is Frobenius, i.e., $\mathfrak{r}^{*}$ has a dense $R$-orbit, which certainly ensures the existence of a generic stabiliser. For $G$ simple, the list of such $V$ is obtained in [15].

Finally, we consider the field of rational invariants for the coadjoint representation of $\mathfrak{r}=\mathfrak{g} \ltimes V$. By [32],

$$
\operatorname{trdeg} \mathbb{k}\left(\mathfrak{r}^{*}\right)^{R}=\operatorname{trdeg} \mathbb{k}\left(V^{*}\right)^{G}+\operatorname{ind} \mathfrak{h},
$$

where $\mathfrak{h}$ is a generic stabiliser for $\left(G: V^{*}\right)$. It follows from Eq. (5.4) that $\mathbb{k}\left(V^{*}\right)^{G}$ can be regarded as a subfield of $\mathbb{k}\left(\mathfrak{r}^{*}\right)^{R}$.

5.10 Theorem. If ind $\mathfrak{h}=0$, then $\mathbb{k}\left(\mathfrak{r}^{*}\right)^{R} \simeq \mathbb{k}\left(V^{*}\right)^{G}$.

Proof. It suffices to verify that $\mathbb{k}\left(V^{*}\right)^{G}$ separates $R$-orbits in a dense open subset of $\mathfrak{r}^{*}$. Let $p: \mathfrak{r}^{*}=V^{*} \oplus \mathfrak{g}^{*} \rightarrow V^{*}$ denote the projection. If $\mathcal{O} \subset V^{*}$ is a generic $G$-orbit, then we will prove that $p^{-1}(\mathcal{O})$ contains a dense $R$-orbit. The latter is equivalent to that, for any $\xi \in \mathcal{O}, G_{\xi} \ltimes V$ has a dense orbit in $p^{-1}(\xi)=$ $\{\xi\} \times \mathfrak{g}^{*}$. Since $1 \ltimes V$ is a normal subgroup of $G_{\xi} \ltimes V$, we first look at its orbits. For any $(\xi, \alpha) \in p^{-1}(\xi)$, we have $(1 \ltimes V) \cdot(\xi, \alpha)=(\xi, \alpha+V * \xi)$. Hence all orbits are parallel affine space of $\operatorname{dimension} \operatorname{dim}(V * \xi)$. Therefore, it will be sufficient to prove that $G_{\xi}$ has a dense orbit in the (geometric) quotient $p^{-1}(\xi) /(1 \ltimes V)$. Because $V * \xi=\left(\mathfrak{g}_{\xi}\right)^{\perp}$, that quotient is isomorphic to $\mathfrak{g}^{*} /\left(\mathfrak{g}_{\xi}\right)^{\perp} \simeq\left(\mathfrak{g}_{\xi}\right)^{*}$ as $G_{\xi}$-variety. Now, the presence of a dense $G_{\xi}$-orbit in $\left(\mathfrak{g}_{\xi}\right)^{*}$ exactly means that ind $\mathfrak{g}_{\xi}=0$, which is true as $\xi$ is generic.

Remarks. 1. In Theorem 5.10, the reductivity of $G$ is not needed. It suffices to assume that $\left(G: V^{*}\right)$ has a generic stabiliser.

2. A related result for $\mathbb{k}\left(\mathfrak{r}^{*}\right)^{R}$ is obtained in [31, Corollary 2.9] under the assumption that $\operatorname{trdeg} \mathbb{k}\left(V^{*}\right)^{G}=0$, but without assuming that $G$ is reductive. 


\section{§6. Reductive Semi-Direct Products and their Polynomial Invariants}

In this section, we study polynomial invariants of semi-direct products $\mathfrak{q}=\mathfrak{g} \ltimes V$, where $\mathfrak{g}$ is reductive.

Our main technical tool is the following result of Igusa (see [19, Lemma 4], [46, Theorem 4.12]). For reader's convenience, we provide a proof. Given an irreducible variety $Y$, we say that an open subset $\Omega \subset Y$ is big if $Y \backslash \Omega$ contains no divisors.

6.1 Lemma (Igusa). Let $A$ be an algebraic group acting regularly on an irreducible affine variety $X$. Suppose $S$ is an integrally closed finitely generated subalgebra of $\mathbb{k}[X]^{A}$ and the morphism $\pi: X \rightarrow \operatorname{Spec} S=: Y$ has the properties:

(i) the fibres of $\pi$ over a dense open subset of $Y$ contain a dense A-orbit;

(ii) $\operatorname{Im} \pi$ contains a big open subset of $Y$.

Then $S=\mathbb{k}[X]^{A}$. In particular, the algebra of A-invariants is finitely generated.

Proof. From (i) and Rosenlicht's theorem, it follows that $\mathbb{k}(Y)=\mathbb{k}(X)^{A}$. In particular, $\mathbb{k}(X)^{A}$ is the quotient field of $\mathbb{k}[X]^{A}$. Assume that $S \neq \mathbb{k}[X]^{A}$. Then one can find a finitely generated intermediate subalgebra: $S \subset \tilde{S} \subset$ $\mathbb{k}[X]^{A}$ such that $S \neq \tilde{S}$. The natural morphism $\tilde{\pi}:$ Spec $\tilde{S} \rightarrow Y$ is birational and its image contains a big open subset of $Y$ (because $\pi$ does). Since $Y$ is normal, the Richardson lemma [5, 3.2 Lemme 1] implies that $\tilde{\pi}$ is an isomorphism. This contradiction shows that $S=\mathbb{k}[X]^{A}$.

Recall that $Q:=G \ltimes V$ is a connected group with Lie algebra $\mathfrak{q}$. Here $1 \ltimes V$ is exactly the unipotent radical of $Q$, which is also denoted $Q^{u}$. Let $T$ be a maximal torus of $G$ with the corresponding Cartan subalgebra $t$.

First, we consider the adjoint representation of $\mathfrak{g} \ltimes V$.

6.2 Theorem. Let $V$ be an arbitrary $G$-module, $\mathfrak{q}=\mathfrak{g} \ltimes V$, and $m=$ $\operatorname{dim} V^{\mathfrak{t}}$. Then

(i) $\mathbb{k}[\mathfrak{q}]^{Q^{u}}$ is a polynomial algebra of Krull dimension $\operatorname{dim} \mathfrak{g}+m$. It is freely generated by the coordinates on $\mathfrak{g}$ and the functions $\widehat{F}_{i}, i=1, \ldots, m$, associated with covariants of type $V^{*}$ (see below).

(ii) $\mathbb{k}[\mathfrak{q}]^{Q}$ is a polynomial algebra of Krull dimension $\operatorname{dim} \mathfrak{t}+m$. It is freely generated by the basic invariants of $\mathbb{k}[\mathfrak{g}]^{G}$ and the same functions $\widehat{F}_{i}, i=$ $1, \ldots, m$. 
(iii) $\max \operatorname{dim}_{x \in \mathfrak{q}} Q \cdot x=\operatorname{dim} \mathfrak{q}-\operatorname{dim} \mathfrak{q} / / Q$;

(iv) If $\pi: \mathfrak{q} \rightarrow \mathfrak{q} / / Q$ is the quotient morphism, then $\Omega:=\left\{x \in \mathfrak{q} \mid d \pi_{x}\right.$ is onto $\}$ is a big open subset of $\mathfrak{q}$.

Proof. (i) By Theorem 4.3, $\operatorname{Mor}_{G}\left(\mathfrak{g}, V^{*}\right)$ is a free $\mathbb{k}[\mathfrak{g}]^{G}$-module of rank $m$. Let $F_{1}, \ldots, F_{m}$ be a basis for this module and $\widehat{F}_{1}, \ldots, \widehat{F}_{m}$ the corresponding $Q$ invariants on $\mathfrak{q}$, i.e., $\widehat{F}_{i}(x+\epsilon v)=\left\langle F_{i}(x), v\right\rangle$. To prove that $\mathbb{k}[\mathfrak{q}]^{Q^{u}}$ is freely generated by the coordinate functions on $\mathfrak{g}$ and the polynomials $\widehat{F}_{i}, i=1, \ldots, m$, we wish to apply Lemma 6.1 .

Set $\mathfrak{X}_{m}=\left\{x \in \mathfrak{g} \mid \operatorname{dim} \operatorname{span}\left\{F_{1}(x), \ldots, F_{m}(x)\right\}=m\right\}$. That is, $\mathfrak{X}_{m}$ is the set of those $x$, where the vectors $F_{i}(x) \in V^{*}, i=1, \ldots, m$, are linearly independent.

Claim. $\mathfrak{X}_{m}$ is a big open subset of $\mathfrak{g}$. More precisely, codim $\mathfrak{g}\left(\mathfrak{g} \backslash \mathfrak{X}_{m}\right) \geqslant 3$.

Proof of the claim. The set of regular elements of $\mathfrak{g}, \mathfrak{g}^{\text {reg }}$, has the property that $\operatorname{codim}\left(\mathfrak{g} \backslash \mathfrak{g}^{r e g}\right) \geqslant 3$ and $\overline{G \cdot x}$ is normal for any $x \in \mathfrak{g}^{r e g}$ [21]. The condition that $\operatorname{codim} \overline{G \cdot x}(\overline{G \cdot x} \backslash G \cdot x) \geqslant 2$ is satisfied for every $x \in \mathfrak{g}$, since any $G$-orbit is even-dimensional. By Theorems 4.2 and 4.3, we conclude that $\mathfrak{X}_{m} \supset \mathfrak{g}^{\text {reg }}$, and the claim follows.

Let $x_{1}, \ldots, x_{n}$ be the coordinates on $\mathfrak{g}$, where $n=\operatorname{dim} \mathfrak{g}$. Then $x_{1}, \ldots, x_{n}$, $\widehat{F}_{1}, \ldots, \widehat{F}_{m}$ are algebraically independent, because their differentials are linearly independent on $\mathfrak{X}_{m} \ltimes V$. Consider the mapping

$$
\tau: \mathfrak{q} \rightarrow \operatorname{Spec} \mathbb{k}\left[x_{1}, \ldots, x_{n}, \widehat{F}_{1}, \ldots, \widehat{F}_{m}\right]=\mathbb{k}^{n+m},
$$

where $\tau(x+\epsilon v)=\left(x, \widehat{F}_{1}(x+\epsilon v), \ldots, \widehat{F}_{m}(x+\epsilon v)\right)$. We identify $\mathbb{k}^{n+m}$ with $\mathfrak{g} \times \mathbb{k}^{m}$. If $x=\left(x_{1}, \ldots, x_{n}\right) \in \mathfrak{X}_{m}$, then the $F_{i}(x)$ 's are linearly independent, so that the system

$$
\widehat{F}_{i}(x+\epsilon v)=\left\langle F_{i}(x), v\right\rangle=\alpha_{i}, \quad i=1, \ldots, m
$$

has a solution $v$ for any $m$-tuple $\alpha=\left(\alpha_{1}, \ldots, \alpha_{m}\right)$. Hence $\operatorname{Im} \tau \supset \mathfrak{X}_{m} \times \mathbb{k}^{m}$, which means that $\operatorname{Im} \tau$ contains a big open subset of $\mathbb{k}^{n+m}$.

It follows from the above Claim that $\mathfrak{g}^{\text {reg }}=\mathfrak{X}_{m} \cap\{y \in \mathfrak{g} \mid \operatorname{dim} G \cdot y=$ $n-m\}$. Take $x \in \mathfrak{g}^{r e g}$, and let $v_{\alpha}$ be a solution to the system $\widehat{F}_{i}(x+\epsilon v)=\alpha_{i}$. Then $\tau^{-1}(x, \alpha) \ni x+\epsilon v_{\alpha}$ and

$$
\tau^{-1}(x, \alpha) \supset Q^{u} \cdot\left(x+\epsilon v_{\alpha}\right)=\left\{x+\epsilon\left(v_{\alpha}+x * V\right)\right\} .
$$

Since $x \in \mathfrak{X}_{m}$, we have $\operatorname{dim} \tau^{-1}(x, \alpha)=n-m$. On the other hand, $\operatorname{dim}[\mathfrak{g}, x]=$ $n-m$, by the definition of $\mathfrak{g}^{r e g}$. Hence $\tau^{-1}(x, \alpha)=Q^{u} \cdot\left(x+\epsilon v_{\alpha}\right)$ for dimension reason. Thus, a generic fibre of $\tau$ is a $Q^{u}$-orbit, and Lemma 6.1 applies here. 
(ii) Clearly,

$$
\mathbb{k}[\mathfrak{q}]^{Q}=\left(\mathbb{k}[\mathfrak{q}]^{Q^{u}}\right)^{G}=\mathbb{k}\left[x_{1}, \ldots, x_{n}, \widehat{F}_{1}, \ldots, \widehat{F}_{m}\right]^{G} .
$$

Since the $\widehat{F}_{i}$ 's are already $G$-invariant, the algebra in question is equal to

$$
\mathbb{k}[\mathfrak{g}]^{G}\left[\widehat{F}_{1}, \ldots, \widehat{F}_{m}\right] .
$$

(iii) The dimension of a $Q$-orbit cannot be greater than $\operatorname{dim} \mathfrak{q}-\operatorname{dim} \mathfrak{q} / / Q$, and if $x \in \mathfrak{t}$ is regular, then $\operatorname{dim} Q \cdot(x+\epsilon 0)=\operatorname{dim} Q-\operatorname{dim} \mathfrak{t}-m$.

(iv) It follows from the previous discussion that $\Omega \supset \mathfrak{g}^{\text {reg }} \ltimes V$.

6.3 Remarks. 1. If $V^{T}=\{0\}$, then the module of covariants of type $V^{*}$ is trivial, so that we obtain a natural isomorphism $\mathbb{k}[\mathfrak{q}]^{Q} \simeq \mathbb{k}[\mathfrak{g}]^{G}$.

2. From Theorem 5.1 and Proposition 5.5, it follows that $\tilde{\mathfrak{t}}:=\mathfrak{t} \ltimes V^{\mathfrak{t}}$ is a generic centraliser in $\mathfrak{q}$ and $\tilde{W}=N_{Q}(\tilde{\mathfrak{t}}) / Z_{Q}(\tilde{\mathfrak{t}})$ is isomorphic to $W=N_{G}(\mathfrak{t}) / Z_{G}(\mathfrak{t})$, the usual Weyl group of $\mathfrak{g}$. Therefore

$$
\mathbb{k}(\mathfrak{q})^{Q} \simeq \mathbb{k}\left(\mathfrak{t} \ltimes V^{\mathfrak{t}}\right)^{W}=\mathbb{k}\left(\mathfrak{t} \times V^{\mathfrak{t}}\right)^{W} .
$$

Since $\mathbb{k}(\mathfrak{t})^{W}$ is a rational field, Theorem 5.1(iv) implies that $\mathbb{k}(\mathfrak{q})^{Q}$ is rational, too. For $\mathfrak{g}$ semisimple, the rationality of $\mathbb{k}(\mathfrak{q})^{Q}$ also follows from Theorem 6.2 , because in this situation $\mathbb{k}(\mathfrak{q})^{Q}$ is the quotient field of $\mathbb{k}[\mathfrak{q}]^{Q}$. However, if $V^{\mathfrak{t}} \neq 0$, then the restriction homomorphism

$$
\text { res }: \mathbb{k}[\mathfrak{q}]^{Q} \rightarrow \mathbb{k}\left[\mathfrak{t} \times V^{\mathfrak{t}}\right]^{W}
$$

is not onto. For, the description of the generators of $\mathbb{k}[\mathfrak{q}]^{Q}$ shows that $\mathbb{k}\left[V^{\mathfrak{t}}\right]^{W}$ does not belong to the image of res.

Now, we look at polynomial invariants of the coadjoint representation of $\mathfrak{q}=$ $\mathfrak{g} \ltimes V$. As we know from $\S 5$, the existence of a generic stabiliser for $\left(\mathfrak{q}, \mathrm{ad}^{*}\right)$ is a rare phenomenon; but this existence is not always needed for describing invariants. It follows from Eq. (5.4) that $\mathbb{k}\left[V^{*}\right]^{G}$ can be regarded as a subalgebra of $\mathbb{k}\left[\mathfrak{q}^{*}\right]^{Q}$. Recall that $\operatorname{trdeg} \mathbb{k}\left(\mathfrak{q}^{*}\right)^{Q}=\operatorname{trdeg} \mathbb{k}\left(V^{*}\right)^{G}+\operatorname{ind} \mathfrak{h}$, where $\mathfrak{h}$ is a generic stabiliser for $\left(G: V^{*}\right)$. In particular, if $\mathfrak{g}$ is semisimple and $\mathfrak{h}$ is reductive, then $\operatorname{trdeg} \mathbb{k}\left(\mathfrak{q}^{*}\right)^{Q}=\operatorname{trdeg} \mathbb{k}\left(V^{*}\right)^{G}+\operatorname{rk} \mathfrak{h}$. Since the roles of $V$ and $\mathfrak{g}$ are interchanged in the dual space, one might hope that $\mathbb{k}\left[\mathfrak{q}^{*}\right]^{Q}$ could be generated by $\mathbb{k}\left[V^{*}\right]^{G}$ and certain invariants arising from $\operatorname{Mor}_{G}\left(V, \mathfrak{g}^{*}\right)$. This is however false, because it can happen that $\mathrm{rk} \mathfrak{h}>0$, but $\operatorname{Mor}_{G}\left(V, \mathfrak{g}^{*}\right)=0$. In general, it is not clear how to discover "missing" invariants associated with the summand ind $\mathfrak{h}$ (or 
$\operatorname{rk} \mathfrak{h})$.

The simplest case is that in which $\mathfrak{h}=0$. Then we are in a position to state an analogue of Theorem 6.2 .

6.4 Theorem. As above, let $\mathfrak{q}=\mathfrak{g} \times V$ and $Q^{u}=1 \ltimes V$. Suppose $a$ generic stabiliser for $\left(G: V^{*}\right)$ is trivial. Then $\mathbb{k}\left[\mathfrak{q}^{*}\right]^{Q^{u}}=\mathbb{k}\left[V^{*}\right]$ and $\mathbb{k}\left[\mathfrak{q}^{*}\right]^{Q}=$ $\mathbb{k}\left[V^{*}\right]^{G}$.

Proof. The second equality stems from the first. To prove the first equality, we use the same method as in Theorem 6.2. The natural projection $\mathfrak{q}^{*} \rightarrow \mathfrak{q}^{*} / \mathfrak{g}^{*} \simeq V^{*}$ is $Q^{u}$-equivariant and satisfies all the requirements of Lemma 6.1. The details are left to the reader.

Remark. In Theorem 6.4, the reductivity of $G$ is not needed.

\section{$\S 7 . \quad$ Takiff Lie Algebras and their Invariants}

For $\mathfrak{g}$ semisimple, some interesting results on the invariants of $(\mathfrak{g} \ltimes \mathfrak{g}$, ad $)$ are obtained by Takiff in [40]. For this reason, Lie algebras of the form $\mathfrak{q} \ltimes \mathfrak{q}$ are sometimes called Takiff (Lie) algebras, see [33],[16]. We will follow this terminology.

In this section, we consider orbits and invariants of certain representations of a Takiff group $\hat{Q}=Q \ltimes \mathfrak{q}$. Some results on rational invariants have already appeared in $\S 5$. Our main object here is the polynomial (regular) invariants. We obtain a generalisation of the main result in [40], which concerns several aspects. First, in place of semisimple Lie algebras, we consider a wider class. Second, the initial representation of $Q$ is not necessarily adjoint. Third, we also describe the invariants of the unipotent group $1 \ltimes \mathfrak{q} \subset \hat{Q}$. Fourth, our proof does not exploit complex numbers and complex topology.

If $V$ is a $\mathfrak{q}$-module, then $V \times V$ can regarded as $\mathfrak{q} \ltimes \mathfrak{q}$-module in a very natural way. For $\left(x_{1}, x_{2}\right) \in \mathfrak{q} \ltimes \mathfrak{q}$ and $\left(v_{1}, v_{2}\right) \in V \times V$, we define

$$
\left(x_{1}, x_{2}\right) \cdot\left(v_{1}, v_{2}\right):=\left(x_{1} \cdot v_{1}, x_{1} \cdot v_{2}-x_{2} \cdot v_{1}\right) .
$$

This $\mathfrak{q}$-module will be denoted by $\hat{V}=V \ltimes V$. We also write $v_{1}+\epsilon v_{2}$ for $\left(v_{1}, v_{2}\right)$. If $f \in \mathbb{k}[V]^{Q}$, then $d f \in \operatorname{Mor}_{Q}\left(V, V^{*}\right)$, and we define $\widehat{F}_{f} \in \mathbb{k}[\hat{V}]$ by the rule: $\widehat{F}_{f}(x+\epsilon y)=\left\langle d f_{x}, y\right\rangle$. Similarly to Lemma 4.6, one proves

$$
\widehat{F}_{f} \in \mathbb{k}[\hat{V}]^{\hat{Q}} .
$$

Here one needs the fact that $d f_{v}$ annihilates the tangent space of $Q \cdot v$ at $v \in V$. 
7.1 Theorem. Let $V$ be a $Q$-module. Suppose the action $(Q: V)$ satisfies the following conditions:

(1) $\mathbb{k}[V]^{Q}$ is a polynomial algebra;

(2) $\max \operatorname{dim}_{v \in V} Q \cdot v=\operatorname{dim} V-\operatorname{dim} V / / Q$;

(3) If $\pi_{Q}: V \rightarrow V / / Q$ is the quotient morphism and $\Omega:=\{v \in V \mid$ $\left(d \pi_{Q}\right)_{v}$ is onto $\}$, then $V \backslash \Omega$ contains no divisors.

Then

(i) $\mathbb{k}[\hat{V}]^{1 \ltimes \mathfrak{q}}$ is a polynomial algebra of Krull dimension $\operatorname{dim} V+\operatorname{dim} V / / Q$, which is generated by the coordinates on the first factor of $\hat{V}$ and the polynomials $\widehat{F}_{1}, \ldots, \widehat{F}_{m}$ associated with the differentials of basic invariants in $\mathbb{k}[V]^{Q}$

(ii) $\mathbb{k}[\hat{V}]^{\hat{Q}}$ is a polynomial algebra of Krull dimension $2 \operatorname{dim} V / / Q$, which is freely generated by the basic invariants of $\mathbb{k}[V]^{Q}$ and the same functions $\widehat{F}_{i}, i=1, \ldots, m$.

(iii) The $\hat{Q}$-module $\hat{V}$ satisfies conditions (1)-(3), too.

Proof. The proof is very close in the spirit to the proof of Theorem 6.2, though some technical details are different.

Set $N=1 \ltimes \mathfrak{q}$. Let $f_{1}, \ldots, f_{m}, m=\operatorname{dim} V / / Q$, be algebraically independent generators of $\mathbb{k}[V]^{Q}$. As was noticed above, to each $f_{i}$ one may associate the polynomial $\widehat{F}_{i}=\widehat{F}_{f_{i}} \in \mathbb{k}[V \ltimes V]^{\hat{Q}}$.

(i) We are going to prove, using Lemma 6.1 , that $\mathbb{k}[\hat{\mathfrak{q}}]^{N}$ is freely generated by the coordinate functions on $V$ (which is the first component of $\hat{V}$ ) and the polynomials $\widehat{F}_{i}, i=1, \ldots, m$. Let $x_{1}, \ldots, x_{n}$ be the coordinate functions on $V$. Then $x_{1}, \ldots, x_{n}, \widehat{F}_{1}, \ldots, \widehat{F}_{m}$ are algebraically independent, because their differentials are linearly independent on $\Omega \ltimes V$. Consider the mapping

$$
\hat{\tau}: \hat{V} \rightarrow \operatorname{Spec} \mathbb{k}\left[x_{1}, \ldots, x_{n}, \widehat{F}_{1}, \ldots, \widehat{F}_{m}\right]=\mathbb{k}^{n+m} .
$$

We identify $\mathbb{k}^{n+m}$ with $V \times \mathbb{k}^{m}$. If $x=\left(x_{1}, \ldots, x_{n}\right) \in \Omega$, then $\left(d f_{i}\right)_{x}$ are linearly independent, so that the system $\widehat{F}_{i}(x+\epsilon y)=\alpha_{i}, i=1, \ldots, m$, has a solution $y$ for any $m$-tuple $\alpha=\left(\alpha_{1}, \ldots, \alpha_{m}\right)$. Hence $\operatorname{Im} \hat{\tau} \supset \Omega \times \mathbb{k}^{m}$, which means that $\operatorname{Im} \hat{\tau}$ contains a big open subset of $\mathbb{k}^{n+m}$.

Next, consider $\Omega^{\prime}=\Omega \cap\{y \in V \mid \operatorname{dim} Q \cdot y=n-m\}$. In view of condition (2), it is still a non-empty open $Q$-stable subset of $V$. Take $x \in \Omega^{\prime}$, and let $y_{\alpha}$ be a solution to the system $\widehat{F}_{i}(x+\epsilon y)=\left\langle\left(d f_{i}\right)_{x}, y\right\rangle=\alpha_{i}, i=1, \ldots, m$. Then $\hat{\tau}^{-1}(x, \alpha) \ni x+\epsilon y_{\alpha}$ and

$$
\hat{\tau}^{-1}(x, \alpha) \supset N \cdot\left(x+\epsilon y_{\alpha}\right)=\left\{x+\epsilon\left(y_{\alpha}+\mathfrak{q} \cdot x\right)\right\} .
$$


Since $x \in \Omega$, we have $\operatorname{dim} \hat{\tau}^{-1}(x, \alpha)=n-m$. On the other hand, $\operatorname{dim} Q \cdot x=$ $n-m$, because of the definition of $\Omega^{\prime}$. Hence $\hat{\tau}^{-1}(x, \alpha)=N \cdot\left(x+\epsilon y_{\alpha}\right)$ for dimension reason. Thus, a generic fibre of $\hat{\tau}$ is an $N$-orbit, and Lemma 6.1 applies here.

(ii) Clearly,

$$
\mathbb{k}[\hat{V}]^{\hat{Q}}=\left(\mathbb{k}[\hat{V}]^{N}\right)^{Q}=\mathbb{k}\left[x_{1}, \ldots, x_{n}, \widehat{F}_{1}, \ldots, \widehat{F}_{m}\right]^{Q} .
$$

Since the $\widehat{F}_{i}$ 's are already $Q$-invariant, the algebra in question is equal to

$$
\mathbb{k}[V]^{Q}\left[\widehat{F}_{1}, \ldots, \widehat{F}_{m}\right]=\mathbb{k}\left[f_{1}, \ldots, f_{m}, \widehat{F}_{1}, \ldots, \widehat{F}_{m}\right] .
$$

(iii) We have to check that the $\hat{Q}$-module $\hat{V}$ satisfies properties (1)-(3).

- Property (1) is verified in (ii).

$\hat{Q}$.

- If $x \in \Omega$, then $\operatorname{dim} \hat{Q} \cdot(x+\epsilon 0)=2 n-2 m$, which gives property (2) for

- Set $\hat{\Omega}=\Omega \times V$. It is a big open subset of $\hat{V}$. Explicit expressions for algebraically independent generators of $\mathbb{k}[\hat{V}]^{\hat{Q}}$ show that their differentials are linearly independent on $\hat{\Omega}$, which is exactly Property (3) for $\hat{V}$.

7.2 Remarks. 1. If the pair $(\mathfrak{q}, V)$ satisfies properties $(1)-(3)$ of Theorem 7.1 , then the passage $(\mathfrak{q}, V) \mapsto(\hat{\mathfrak{q}}, \hat{V})$ can be iterated ad infinitum without losing those properties.

2. Since the adjoint representation of a semisimple Lie algebra $\mathfrak{g}$ has properties (1)-(3), iterating the Takiffisation procedure $\mathfrak{g} \mapsto \mathfrak{g} \ltimes \mathfrak{g}$ always yields algebras with a polynomial ring of invariants for the adjoint representation. This is the main result of [40]. Explicit form of the basic invariants for $(\mathfrak{g} \ltimes \mathfrak{g}$, ad $)$ is also pointed out there. Notice also that Takiff's results follow from either Theorem 6.2 with $V=\mathfrak{g}$ or Theorem 7.1 with $\mathfrak{q}=V=\mathfrak{g}$.

3. By Theorem 6.2, the adjoint representation of $\mathfrak{q}=\mathfrak{g} \ltimes V$ satisfies all the conditions of Theorem 7.1. Therefore these $\mathfrak{q}$ can be used as building blocks for Takiffisation procedure, which yields more and more complicated Lie algebras having polynomial algebras of invariants.

Let us make some comments on the conditions of Theorem 7.1. If $Q=G$ is semisimple, then conditions (2) and (3) are always satisfied, regardless of the fact whether $\mathbb{k}[V]^{G}$ is polynomial. For condition (3) we refer to [20, Satz 2], while (2) follows since $G$ has no rational characters and therefore $\mathbb{k}(V)^{G}$ is the quotient field of $\mathbb{k}[V]^{G}$. Thus, we have

7.3 Corollary. If $\rho: G \rightarrow G L(V)$ is a representation of a semisimple group such that $\mathbb{k}[V]^{G}$ is polynomial, then Theorem 7.1 applies to the $G \ltimes \mathfrak{g}$ module $V \ltimes V$. 


\section{$\S 8 . \quad$ The Null-Cone and its Irreducibility}

In previous sections, we described several instances of representations of nonreductive Lie algebras having a polynomial algebra of invariants. If $Q \subset$ $G L(\tilde{V})$ and $\mathbb{k}[\tilde{V}]^{Q}$ is polynomial, then it is natural to inquire of whether it is true that $\mathbb{k}[\tilde{V}]$ is a free $\mathbb{k}[\tilde{V}]^{Q}$-module. As is well known, the freeness is equivalent to that the quotient morphism $\pi: \tilde{V} \rightarrow \tilde{V} / / Q$ is equidimensional, i.e., has the property that $\operatorname{dim} \pi^{-1}(\pi(0))=\operatorname{dim} \tilde{V}-\operatorname{dim} \tilde{V} / / Q$. As in the case of reductive group actions, we say that $\pi^{-1}(\pi(0))$ is the null-cone, denoted $\mathcal{N}^{Q}(\tilde{V})$ or $\mathcal{N}(\tilde{V})$.

In this section, we only deal with reductive semi-direct products and their representations. Our goal is to describe necessary and sufficient conditions for equidimensionality of $\pi$ and point out some consequences of it. We consider two types of representations:

A) $\mathfrak{q}=\mathfrak{g} \ltimes V$, where $V$ is a $\mathfrak{g}$-module, and $\tilde{V}=\mathfrak{q}$, i.e., we consider the adjoint representation of $\mathfrak{q}$.

B) $\mathfrak{q}=\mathfrak{g} \ltimes \mathfrak{g}$ is a reductive Takiff algebra and $\tilde{V}=V \ltimes V$, where $V$ is a $\mathfrak{g}$-module.

We begin with case $\mathbf{A})$. Recall that $m=\operatorname{dim} V^{T}$ and $F_{1}, \ldots, F_{m}$ is a basis for the $\mathbb{k}[\mathfrak{g}]^{G}$-module $\operatorname{Mor}_{G}\left(\mathfrak{g}, V^{*}\right)$. The null-cone for $(\mathfrak{g}$, ad $)$ is denoted by $\mathcal{N}(\mathfrak{g})$ or merely by $\mathcal{N}$. In other words, $\mathcal{N}$ is the set of nilpotent elements of $\mathfrak{g}$. Recall that $\mathcal{N}$ is irreducible and $\operatorname{dim} \mathcal{N}=\operatorname{dim} \mathfrak{g}-\operatorname{dim} \mathfrak{g} / / G=\operatorname{dim} \mathfrak{g}-\operatorname{dim} \mathfrak{t}$.

Theorem 6.2 says that if $V^{T}=0$, then $\mathbb{k}[\mathfrak{q}]^{Q}=\mathbb{k}[\mathfrak{g}]^{G}$ and therefore $\mathcal{N}(\mathfrak{q}) \simeq \mathcal{N} \times$ $V$. In this trivial case, $\pi_{Q}$ is equidimensional, since it is so for $\pi_{G}: \mathfrak{g} \rightarrow \mathfrak{g} / / G$. Therefore we assume below that $V^{T} \neq 0$.

Define a stratification of $\mathfrak{g}$ in the following way:

$$
\mathfrak{X}_{i, V}=\mathfrak{X}_{i}=\left\{x \in \mathfrak{g} \mid \operatorname{dim} \operatorname{span}\left\{F_{1}(x), \ldots, F_{m}(x)\right\}=i\right\} .
$$

Then $\overline{\mathfrak{X}}_{i} \subset \overline{\mathfrak{X}}_{i+1}$ and $\overline{\mathfrak{X}}_{m}=\mathfrak{g}$. The induced stratification on the null-cone is $\mathfrak{X}_{i}(\mathcal{N}):=\mathfrak{X}_{i} \cap \mathcal{N}$. As is shown in the proof of Theorem 6.2, $\mathfrak{X}_{m}$ is a big open subset of $\mathfrak{g}$ containing $\mathfrak{g}^{\text {reg }}$. Therefore $\mathfrak{X}_{m}(\mathcal{N})$ is a big open subset of $\mathcal{N}$ containing the regular nilpotent orbit.

\subsection{Theorem.}

1. The quotient morphism $\pi_{Q}: \mathfrak{q} \rightarrow \mathfrak{q} / / Q$ is equidimensional if and only if $\operatorname{codim}_{\mathcal{N}} \mathfrak{X}_{i}(\mathcal{N}) \geqslant m-i$.

2. If $\mathcal{N}(\mathfrak{q})$ is irreducible, then $\pi_{Q}$ is equidimensional;

3. $\mathcal{N}(\mathfrak{q})$ is irreducible if and only if $\operatorname{codim}_{\mathcal{N}} \mathfrak{X}_{i}(\mathcal{N}) \geqslant m-i+1$ for $i<m$. 
Proof. 1. Since $\pi_{Q}$ is dominant, all irreducible components of $\mathcal{N}(\mathfrak{q})$ are of dimension $\geqslant \operatorname{dim} \mathfrak{q}-\operatorname{dim} \mathfrak{q} / / Q$. By Theorem $6.2, \mathcal{N}(\mathfrak{q})=\{(x, v) \mid x \in$ $\left.\mathcal{N} \& \widehat{F}_{i}(x, v)=\left\langle F_{i}(x), v\right\rangle=0 \quad \forall i\right\}$. Let $p: \mathcal{N}(\mathfrak{q}) \rightarrow \mathcal{N}$ be the projection onto the first factor. Then $\mathcal{N}(\mathfrak{q})=\bigsqcup_{i=0}^{m} p^{-1}\left(\mathfrak{X}_{i}(\mathcal{N})\right)$ and $\operatorname{dim} p^{-1}\left(\mathfrak{X}_{i}(\mathcal{N})\right)=$ $\operatorname{dim} \mathfrak{X}_{i}(\mathcal{N})+\operatorname{dim} V-i$.

2. By Theorem 6.2, if $e \in \mathcal{N}^{r e g}$, then $(e, 0) \in \mathfrak{q}^{r e g}$ and $\left(d \pi_{Q}\right)_{(e, 0)}$ is onto. Therefore, $(e, 0)$ is a smooth point of $\mathcal{N}(\mathfrak{q})$, and the unique irreducible component of $\mathcal{N}(\mathfrak{q})$ to which $(e, 0)$ belongs is of dimension $\operatorname{dim} \mathfrak{q}-\operatorname{dim} \mathfrak{q} / / Q$. On the other hand, $\operatorname{dim} p^{-1}\left(\mathfrak{X}_{m}(\mathcal{N})\right)=\operatorname{dim} \mathcal{N}+\operatorname{dim} V-m=\operatorname{dim} \mathfrak{q}-\operatorname{dim} \mathfrak{q} / / Q$. Hence $\overline{p^{-1}\left(\mathfrak{X}_{m}(\mathcal{N})\right)}$ is the irreducible component of $\mathcal{N}(\mathfrak{q})$ containing $(e, 0)$.

3. The proof of part 2 shows that $\overline{p^{-1}\left(\mathfrak{X}_{m}(\mathcal{N})\right)}$ is an irreducible component of $\mathcal{N}(\mathfrak{q})$ of expected dimension. To ensure the irreducibility, we have to require that $\overline{p^{-1}\left(\mathfrak{X}_{i}(\mathcal{N})\right)}$ cannot be an irreducible component for $i<m$. Since all irreducible components of $\mathcal{N}(\mathfrak{q})$ are of dimension $\geqslant \operatorname{dim} \mathfrak{q}-\operatorname{dim} \mathfrak{q} / / Q$, the condition that $\operatorname{dim} p^{-1}\left(\mathfrak{X}_{i}(\mathcal{N})\right)<\operatorname{dim} \mathfrak{q}-\operatorname{dim} \mathfrak{q} / / Q$ for $i<m$ is equivalent to the irreducibility.

The following is now immediate.

8.2 Corollary. If $m=1$, then $\mathcal{N}(\mathfrak{q})$ is irreducible; if $m=2$, then $\pi_{Q}$ is equidimensional.

8.3 Remarks. 1. Since $\mathcal{N}$ consists of finitely many $G$-orbits, condition 8.1(1) is equivalent to the following: if $G \cdot x \subset \mathfrak{X}_{i}(\mathcal{N})$, then $\operatorname{dim} G \cdot x \leqslant$ $\operatorname{dim} \mathcal{N}-(m-i)$, or

$$
\operatorname{dim} \mathfrak{z}_{\mathfrak{g}}(x)-\operatorname{rk} \mathfrak{g} \geqslant m-\operatorname{dim}\left(\operatorname{span}\left\{F_{1}(x), \ldots, F_{m}(x)\right\}\right) .
$$

Furthermore, a more careful look at the projection $\mathcal{N}(\mathfrak{q}) \rightarrow \mathcal{N}$ shows that if last condition is satisfied, then the number of the irreducible components of $\mathcal{N}(\mathfrak{q})$ equals the number of the $G$-orbits $G \cdot x \subset \mathcal{N}$ such that $\operatorname{dim} \mathfrak{z} \mathfrak{g}(x)-\operatorname{rk} \mathfrak{g}=$ $m-\operatorname{dim}\left(\operatorname{span}\left\{F_{1}(x), \ldots, F_{m}(x)\right\}\right)$.

2. The condition in Theorem 8.1(1) for $i=0$ reads $\operatorname{dim} \mathcal{N}-\operatorname{dim}\left(\mathfrak{X}_{0}(\mathcal{N})\right) \geqslant$ $m$, or $\operatorname{dim} V^{T} \leqslant \operatorname{dim} \mathcal{N}-\operatorname{dim} \mathfrak{X}_{0}(\mathcal{N}) \leqslant \operatorname{dim} \mathcal{N}$. This is a rough necessary condition for $\pi_{Q}$ to be equidimensional. Let $G$ be simple and $V_{\lambda}$ a simple $G$ module with highest weight $\lambda$. Then $\left(V_{\lambda}\right)^{T} \neq 0$ if and only if $\lambda$ lies in the root lattice, $\mathcal{R}$. The function $n \mapsto \operatorname{dim}\left(V_{n \lambda}\right)^{T}, \lambda \in \mathcal{R}$, has a polynomial growth. The only case in which this function is constant is that of $G=S L_{p}, \lambda=p \varphi_{1}$ or $p \varphi_{p-1}$. Here $\varphi_{i}$ 's are fundamental weights, and $\operatorname{dim}\left(V_{n \lambda}\right)^{T}=1$ for any $n \in \mathbb{N}$. 
Thus, modulo this exception, there are finitely many simple $G$-modules $V$ such that $V^{T} \neq 0$ and $\pi_{Q}$ is equidimensional.

For future use, we record a relationship between the stratifications of $\mathcal{N}$ and $\mathfrak{g}$.

8.4 Proposition. If $\operatorname{codim}_{\mathcal{N}} \mathfrak{X}_{i}(\mathcal{N}) \geqslant m-i+1$ for $i<m$, then $\operatorname{codim}_{\mathfrak{g}} \mathfrak{X}_{i} \geqslant m-i+2$.

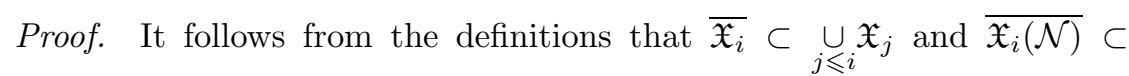
$\cup_{j \leqslant i} \mathfrak{X}_{j}(\mathcal{N})$. For $i<m$, we have $\mathfrak{X}_{i} \cap \mathfrak{g}^{\text {reg }}=\varnothing$ and hence $\pi_{G}\left(\overline{\mathfrak{X}_{i}}\right)=\overline{\mathfrak{X}_{i}} / / G$ is a proper subvariety of $\mathfrak{g} / / G$. Therefore

$\operatorname{dim} \overline{\mathfrak{X}_{i}} \leqslant \operatorname{dim} \mathfrak{g} / / G-1+\operatorname{dim} \overline{\mathfrak{X}_{i}(\mathcal{N})} \leqslant \operatorname{dim} \mathfrak{g}-(m-i+2)$.

There is another interesting cone related to $\mathfrak{q}=\mathfrak{g} \ltimes V$. Consider the morphism $\bar{\pi}: \mathfrak{q} \rightarrow \mathbb{k}^{m},(x, v) \mapsto\left(\widehat{F}_{1}(x, v), \ldots, \widehat{F}_{m}(x, v)\right)$. The zero-fibre of $\bar{\pi}$ is denoted by $\mathcal{N}^{u}(\mathfrak{q})$. Thus,

$$
\mathcal{N}^{u}(\mathfrak{q})=\left\{(x, v) \in \mathfrak{q} \mid\left\langle F_{i}(x), v\right\rangle=0 \quad i=1, \ldots, m\right\} .
$$

The proof of the following result is entirely similar to that of Theorem 8.1. One should only consider the projection $\mathcal{N}^{u}(\mathfrak{q}) \rightarrow \mathfrak{g}$.

\subsection{Theorem.}

1. The morphism $\bar{\pi}: \mathfrak{q} \rightarrow \mathbb{k}^{m}$ is equidimensional if and only if $\operatorname{codim}_{\mathfrak{g}} \mathfrak{X}_{i} \geqslant$ $m-i$.

2. If $\mathcal{N}^{u}(\mathfrak{q})$ is irreducible, then $\bar{\pi}$ is equidimensional;

3. $\mathcal{N}^{u}(\mathfrak{q})$ is irreducible if and only if $\operatorname{codim}_{\mathfrak{g}} \mathfrak{X}_{i} \geqslant m-i+1$ for $i<m$.

Now, comparing Theorem 8.1(iii), Proposition 8.4, and Theorem 8.5(iii), one concludes that if $\mathcal{N}(\mathfrak{q})$ is irreducible, then so is $\mathcal{N}^{u}(\mathfrak{q})$.

But one can derive a much stronger assertion on $\mathcal{N}^{u}(\mathfrak{q})$ from the irreducibility of $\mathcal{N}(\mathfrak{q})$. This is related to properties of symmetric algebras of certain modules over polynomial rings and exploits some technique from [22], [27].

Let $\operatorname{Mor}\left(\mathfrak{g}, V^{*}\right)$ be the $\mathbb{k}[\mathfrak{g}]$-module of all polynomial morphisms $F: \mathfrak{g} \rightarrow$ $V^{*}$. Consider the homomorphism $\hat{\tau}: \operatorname{Mor}\left(\mathfrak{g}, V^{*}\right) \rightarrow \operatorname{Mor}\left(\mathfrak{g}, V^{*}\right)$ defined by $\hat{\tau}(F)(x)=x \cdot F(x)$. (Here "." refers to the $\mathfrak{g}$-module structure on $V^{*}$.)

8.6 Theorem. $\operatorname{ker} \hat{\tau}$ is a free $\mathbb{k}[\mathfrak{g}]$-module of rank $m$. More precisely, $\left(F_{1}, \ldots, F_{m}\right)$ is a basis for ker $\hat{\tau}$. 
Proof. The proof is based on the same idea as the proof of Theorem 1.9 in [27].

Clearly, $\operatorname{ker} \hat{\tau}$ is a torsion-free $\mathbb{k}[\mathfrak{g}]$-module and the $\operatorname{rank} \operatorname{rk}(\operatorname{ker} \hat{\tau}):=$ $\operatorname{dim}\left(\operatorname{ker} \hat{\tau} \otimes_{\mathbb{k}[\mathfrak{g}]} \mathbb{k}(\mathfrak{g})\right)$ is well-defined. An easy argument shows that the rank of $\hat{\tau}$ over $\mathbb{k}(\mathfrak{g})$ equals $\operatorname{dim} V-\max _{x \in \mathfrak{g}} \operatorname{dim}\left(V^{*}\right)^{x}=\operatorname{dim} V-m$. Hence $\operatorname{rk}(\operatorname{ker} \hat{\tau})=m$. Obviously, $F_{i} \in \operatorname{ker} \hat{\tau}$ and $\bigoplus_{i=1}^{m} \mathbb{k}[\mathfrak{g}] F_{i}$ is a free submodule of ker $\hat{\tau}$ of rank $m$. It follows that, for any $F \in \operatorname{ker} \hat{\tau}$, there exist $\hat{p}, p_{1}, \ldots, p_{m} \in \mathbb{k}[\mathfrak{g}]$ such that

$$
\hat{p} F=\sum_{i} p_{i} F_{i}
$$

Assume $\hat{p} \notin \mathbb{k}^{*}$. Let $p$ be a prime factor of $\hat{p}$ and $D$ the divisor of zeros of $p$. Then $\sum_{i} p_{i}(v) F_{i}(v)=0$ for any $v \in D$. Since $\mathfrak{g}^{\text {reg }}$ is big, $\mathfrak{g}^{\text {reg }} \cap D$ is dense in $D$. Because $\left\{F_{i}(v)\right\}$ are linearly independent for $v \in \mathfrak{g}^{\text {reg }}$, we obtain $\left.p_{i}\right|_{D} \equiv 0$. Hence $p_{i} / p \in \mathbb{k}[\mathfrak{g}]$ for each $i$, and we are done.

Let $E$ denote the $\mathbb{k}[\mathfrak{g}]$-module $\operatorname{Im} \hat{\tau}$. In view of the previous theorem, we have the exact sequence

$$
0 \rightarrow \bigoplus_{i=1}^{m} \mathbb{k}[\mathfrak{g}] F_{i} \stackrel{\hat{\beta}}{\rightarrow} \operatorname{Mor}\left(\mathfrak{g}, V^{*}\right) \stackrel{\hat{T}}{\rightarrow} E \rightarrow 0
$$

Choose a basis $\xi_{1}, \ldots, \xi_{n}$ for $V^{*}$. Using this basis, we identify $\operatorname{Mor}\left(\mathfrak{g}, V^{*}\right)=$ $\mathbb{k}[\mathfrak{g}] \otimes V^{*}$ with $\mathbb{k}[\mathfrak{g}]^{n}$. Then we can write $F_{j}(x)=\sum_{i=1}^{n} F_{i j}(x) \xi_{i}$, where $F_{i j} \in$ $\mathbb{k}[\mathfrak{g}]$. If we regard sequence $(8.7)$ as a sequence

$$
0 \rightarrow \mathbb{k}[\mathfrak{g}]^{m} \stackrel{\hat{\beta}}{\rightarrow} \mathbb{k}[\mathfrak{g}]^{n} \stackrel{\hat{\tau}}{\rightarrow} E \rightarrow 0
$$

then $\hat{\beta}$ becomes an $n \times m$-matrix with entries $F_{i j}$. Let $I_{t}(\hat{\beta})$ be the ideal generated by $t \times t$ minors of $\hat{\beta}$. For $d \in \mathbb{N}$, consider the following condition

$$
\text { ht } I_{t}(\hat{\beta}) \geqslant m-t+1+d \quad \text { for } 1 \leqslant t \leqslant m \text {. }
$$

The ideals $I_{t}(\hat{\beta})$ are independent of the presentation of $E$. These are Fitting ideals of $E$, see e.g. $[43,1.1]$. Let $\operatorname{Sym}_{\mathbb{k}[\mathfrak{g}]}(E)$ denote the symmetric algebra of the $\mathbb{k}[\mathfrak{g}]$-module $E$.

8.8 Theorem. Suppose $\mathcal{N}(\mathfrak{q})$ is irreducible. Then

(i) The condition $\left(\mathcal{F}_{2}\right)$ is satisfied by $E$.

(ii) $\operatorname{Sym}_{\mathbb{k}[\mathfrak{g}]}(E)$ is a factorial domain of Krull dimension $\operatorname{dim} \mathfrak{g}+n-m$. 
(iii) $\mathcal{N}^{u}(\mathfrak{q})$ is an irreducible factorial complete intersection, and $\mathbb{k}\left[\mathcal{N}^{u}(\mathfrak{q})\right]=$ $\operatorname{Sym}_{\mathrm{k}[\mathfrak{g}]} E$.

(iv) $\mathcal{N}^{u}(\mathfrak{q})=\overline{\operatorname{Im}(\kappa)}$, where $\kappa: \mathfrak{q} \rightarrow \mathfrak{q}$ is defined by $\kappa(x, v)=(x, x \cdot v), x \in$ $\mathfrak{g}, v \in V$.

Proof. (i) It is easily seen that $\mathfrak{X}_{i}$ is the zero locus of $I_{i+1}(\hat{\beta})$. Therefore condition $\left(\mathcal{F}_{2}\right)$ is satisfied in view of Proposition 8.4.

(ii) The exact sequence (8.7) shows that $E$ has projective dimension at most one. Therefore part (ii) follows from (i) combined with [2, Prop. $3 \& 6]$.

(iii) The universal property of symmetric algebras implies that $\operatorname{Sym}_{\mathbb{k}[\mathfrak{g}]}(E)$ is the quotient of $\operatorname{Sym}_{\mathbb{k}[\mathfrak{g}]}\left(\mathbb{k}[\mathfrak{g}] \otimes V^{*}\right)=\mathbb{k}[\mathfrak{g} \times V]$ by the ideal generated by the image of $\hat{\beta}$. It follows from the construction that $\hat{\beta}\left(F_{i}\right)=\widehat{F}_{i}$. Hence $\operatorname{Sym}_{\mathbb{k}[\mathfrak{g}]}(E)=\mathbb{k}\left[\mathcal{N}^{u}(\mathfrak{q})\right]$, and the other assertions follow from (ii).

(iv) Clearly, $\overline{\operatorname{Im}(\kappa)}$ is an irreducible subvariety of $\mathfrak{q}$. Taking the (surjective) projection to $\mathfrak{g}$ and looking at the dimension of the generic fibre, one finds that $\operatorname{dim} \overline{\operatorname{Im}(\kappa)}=\operatorname{dim} \mathfrak{g}+n-m$. Thus, $\overline{\operatorname{Im}(\kappa)} \subset \mathcal{N}^{u}(\mathfrak{q})$, both have the same dimension and are irreducible. Hence they are equal.

Remark. For $V=\mathfrak{g}$, i.e., for the Takiff algebra $\mathfrak{g} \ltimes \mathfrak{g}$, condition $\left(\mathcal{F}_{2}\right)$ can be proved directly, without referring to the irreducibility of $\mathcal{N}(\mathfrak{q})$, see $[22$, Prop. 2.1]. In this special case, the above results for $\mathcal{N}^{u}(\mathfrak{q})$ are already obtained in [22, Prop. 2.4]. Actually, $\mathcal{N}(\mathfrak{q})$ is irreducible if $V=\mathfrak{g}$. But this fact, as well as "Takiff" terminology, was not used in loc. cit. In $\S 9$, we give new examples of semi-direct products $\mathfrak{q}=\mathfrak{g} \ltimes V$ such that $\mathcal{N}(\mathfrak{q})$ is irreducible and thereby new instances, where Theorem 8.8 applies.

Now, we proceed to case $\mathbf{B})$.

Recall that $\hat{G}=G \ltimes \mathfrak{g}$ and $\hat{V}=V \ltimes V$ is a $\hat{G}$-module. To a great extent, our results in this case are similar to those in case A). A notable distinction is, however, that whereas the adjoint representation of $G$ has some good properties for granted, we have to require these properties for $(G: V)$.

We will assume below that $(G: V)$ satisfies properties (1)-(3) of Theorem 7.1, with $G$ in place of $Q$, and use the respective notation. In particular, $\hat{G}^{u}=1 \ltimes V, m=\operatorname{dim} V / / G, \mathbb{k}[V]^{G}=\mathbb{k}\left[f_{1}, \ldots, f_{m}\right]$, and $\widehat{F}_{i} \in \mathbb{k}[\hat{V}]^{\hat{G}}$ is the invariant associated with $d f_{i}$. As in case A), we define a stratification of $V$ by

$\mathfrak{Y}_{i}=\left\{x \in V \mid \operatorname{dim} \operatorname{span}\left\{\left(d f_{1}\right)_{x}, \ldots,\left(d f_{m}\right)_{x}\right\}=i\right\}=\left\{x \in V \mid \operatorname{rk}\left(d \pi_{G}\right)_{x}=i\right\}$.

Then $\overline{\mathfrak{Y}}_{i} \subset \overline{\mathfrak{Y}}_{i+1}$ and $\overline{\mathfrak{Y}}_{m}=V$. Notice that $\mathfrak{Y}_{0}=\{0\}$. The induced stratification of the null-cone $\mathcal{N}^{G}(V)=\mathcal{N}(V)$ is $\mathfrak{Y}_{i}(\mathcal{N}(V)):=\mathfrak{Y}_{i} \cap \mathcal{N}(V)$. Since 
$\pi_{G}: V \rightarrow V / / G$ is onto, $\operatorname{dim} \mathcal{N}(V) \geqslant \operatorname{dim} V-\operatorname{dim} V / / G$. But, unlike the case of $(G: \mathfrak{g})$, it may happen that the last inequality is strict and $\mathfrak{Y}_{m}(\mathcal{N}(V))=\varnothing$.

8.9 Lemma. Suppose $\pi_{\hat{G}}: \hat{V} \rightarrow \hat{V} / / \hat{G}$ is equidimensional. Then so is $\pi_{G}: V \rightarrow V / / G$ and $\mathfrak{Y}_{m}(\mathcal{N}(V)) \neq \varnothing$.

Proof. Consider the projection $p: \mathcal{N}(\hat{V}) \rightarrow \mathcal{N}(V)$. If $j$ is the maximal index such that $\mathfrak{Y}_{j}(\mathcal{N}(V)) \neq \varnothing$, then $\operatorname{dim} \mathcal{N}(\hat{V})=\operatorname{dim} \mathcal{N}(V)+\operatorname{dim} V-j$. Since $\operatorname{dim} \mathcal{N}(V) \geqslant \operatorname{dim} V-m$, the result follows.

Thus, if we are searching for equidimensional quotient morphisms $\pi_{\hat{G}}$, then we must assume that

$$
\operatorname{dim} \mathcal{N}(V)=\operatorname{dim} V-\operatorname{dim} V / / G \text { and } \mathfrak{Y}_{m}(\mathcal{N}(V)) \neq \varnothing
$$

In this setting, analogs of results (8.1)-(8.8) are proved in a quite similar fashion. Let $\mathcal{N}^{u}(\hat{V})$ denote the zero-fibre of the morphism $\bar{\pi}: \hat{V} \rightarrow \mathbb{k}^{m}$ defined by

$$
\bar{\pi}\left(v_{1}, v_{2}\right)=\left(\widehat{F}_{1}\left(v_{1}, v_{2}\right), \ldots, \widehat{F}_{m}\left(v_{1}, v_{2}\right)\right)=\left(\left\langle\left(d f_{1}\right)_{v_{1}}, v_{2}\right\rangle, \ldots,\left\langle\left(d f_{m}\right)_{v_{1}}, v_{2}\right\rangle\right) .
$$

8.10 Theorem. Under the assumptions (1)-(3) of Theorem 7.1 and (*), we have

1. The morphism $\pi_{\hat{G}}: \hat{V} \rightarrow \hat{V} / / \hat{G}$ (resp. $\bar{\pi}: \hat{V} \rightarrow \mathbb{k}^{m}$ ) is equidimensional if and only if $\operatorname{codim}_{\mathcal{N}(V)} \mathfrak{Y}_{i}(\mathcal{N}(V)) \geqslant m-i\left(\right.$ resp. $\left.\operatorname{codim}_{V} \mathfrak{Y}_{i} \geqslant m-i\right)$ for all $i$.

2. If $\mathcal{N}(\hat{V})$ (resp. $\left.\mathcal{N}^{u}(\hat{V})\right)$ is irreducible, then $\pi_{\hat{G}}$ (resp. $\left.\bar{\pi}\right)$ is equidimensional;

3. $\mathcal{N}(\hat{V})$ (resp. $\left.\mathcal{N}^{u}(\hat{V})\right)$ is irreducible if and only if $\operatorname{codim}_{\mathcal{N}(V)} \mathfrak{Y}_{i}(\mathcal{N}(V)) \geqslant$ $m-i+1\left(\right.$ resp. $\left.\operatorname{codim}_{V} \mathfrak{Y}_{i} \geqslant m-i+1\right)$ for $i<m$.

4. If $\operatorname{codim}_{\mathcal{N}(V)} \mathfrak{Y}_{i}(\mathcal{N}(V)) \geqslant a$, then $\operatorname{codim}_{V} \mathfrak{Y}_{i} \geqslant m-i+a$.

Proof. The proof of parts 1-3 is similar to the proof of Theorem 8.1. For the last part, we notice that $\operatorname{dim} \overline{\mathfrak{Y}}_{i} / / G \leqslant i$. Therefore $\operatorname{dim} \mathfrak{Y}_{i} \leqslant i+$ $\operatorname{dim} \mathfrak{Y}_{i}(\mathcal{N}(V)) \leqslant i+\operatorname{dim} \mathcal{N}(V)-a=\operatorname{dim} V-(m-i+a)$. (Cf. the proof of Prop. 2.1 in [22].)

Consider the homomorphism of $\mathbb{k}[V]$-modules

$$
\hat{\mu}: \operatorname{Mor}\left(V, V^{*}\right) \rightarrow \operatorname{Mor}\left(V, \mathfrak{g}^{*}\right)
$$


defined by $\langle\hat{\mu}(F)(v), s\rangle:=\langle F(v), s \cdot v\rangle$ for $v \in V, s \in \mathfrak{g}$. Here "." refers to the $\mathfrak{g}$-module structure on $V$ and the first (resp. second) $\langle$,$\rangle stands for the pairing$ of $\mathfrak{g}$ and $\mathfrak{g}^{*}$ (resp. $V$ and $V^{*}$ ). By [27, Theorem 1.9], ker $\hat{\mu}$ is a free $\mathbb{k}[V]$-module of rank $m$ generated by $d f_{i}, i=1, \ldots, m$. Let $\hat{E}$ denote the $\mathbb{k}[V]$-module $\operatorname{Im} \hat{\mu}$.

8.11 Theorem. Suppose $\mathcal{N}(\hat{V})$ is irreducible. Then

(i) The condition $\left(\mathcal{F}_{2}\right)$ is satisfied by $\hat{E}$.

(ii) $\operatorname{Sym}_{\mathbb{R}[V]}(\hat{E})$ is a factorial domain of Krull dimension $2 \operatorname{dim} V-m$.

(iii) $\mathcal{N}^{u}(\hat{V})$ is an irreducible factorial complete intersection, and $\mathbb{k}\left[\mathcal{N}^{u}(\hat{V})\right]=$ $\operatorname{Sym}_{\mathbb{k}[V]} \hat{E}$.

(iv) $\mathcal{N}^{u}(\hat{V})=\overline{\operatorname{Im}(\varkappa)}$, where $\varkappa: V \times \mathfrak{g} \rightarrow V \times V$ is defined by $\varkappa(v, x)=(v, x \cdot v)$, $x \in \mathfrak{g}, v \in V$.

The proof of Theorem 8.11 is omitted, since it is similar to the proof of Theorem 8.8.

\section{$\S 9$. Isotropy Contractions and $\mathbb{Z}_{2}$-Contractions of Semisimple Lie} Algebras

Let $\mathfrak{h}$ be a subalgebra of $\mathfrak{q}$ such that $\mathfrak{q}=\mathfrak{h} \oplus \mathfrak{m}$ for some ad $\mathfrak{h}$-stable subspace $\mathfrak{m} \subset \mathfrak{q}$. (Such an $\mathfrak{h}$ is said to be reductive in $\mathfrak{q}$.) For instance, if $\vartheta$ is an involutory automorphism of $\mathfrak{q}$, then +1 and -1 -eigenspaces of $\vartheta$ yield such a decomposition. The fixed-point subalgebra of an involutory automorphism is called a symmetric subalgebra.

9.1 Definition. If $\mathfrak{h}$ is reductive in $\mathfrak{q}$, then the semi-direct product $\mathfrak{h} \ltimes \mathfrak{m}$ is called an isotropy contraction of $\mathfrak{q}$. If $\mathfrak{h}$ is symmetric, so the decomposition $\mathfrak{q}=\mathfrak{h} \oplus \mathfrak{m}$ is a $\mathbb{Z}_{2}$-grading, then $\mathfrak{h} \ltimes \mathfrak{m}$ is also called a $\mathbb{Z}_{2}$-contraction of $\mathfrak{q}$.

Notice that $\mathfrak{h} \ltimes \mathfrak{m}$ is a contraction of $\mathfrak{q}$ in the sense of the deformation theory of Lie algebras, see e.g. [45, Chapter $7, \S 2]$. More precisely, consider the invertible linear map $c_{t}: \mathfrak{q} \rightarrow \mathfrak{q}, t \in \mathbb{k} \backslash\{0\}$, such that $c_{t}(h+m)=h+t^{-1} m$. Define the new Lie algebra multiplication $[,]_{(t)}$ on the vector space $\mathfrak{q}$ by the rule

$$
[x, y]_{(t)}:=c_{t}\left(\left[c_{t}^{-1}(x), c_{t}^{-1}(y)\right]\right), \quad x, y \in \mathfrak{q} .
$$

Then, for all $t \neq 0$, the algebras $\mathfrak{q}_{(t)}$ are isomorphic, and $\lim _{t \rightarrow 0} \mathfrak{q}_{(t)}=\mathfrak{h} \ltimes \mathfrak{m}$.

9.2 Lemma. Any Takiff Lie algebra is a $\mathbb{Z}_{2}$-contraction. 
Proof. Consider the direct sum of Lie algebras $\mathfrak{q} \dot{+} \mathfrak{q}$ and the involution $\vartheta$ permuting the summands. Then the corresponding $\mathbb{Z}_{2}$-contraction is isomorphic to $\mathfrak{q} \ltimes \mathfrak{q}$.

In the rest of the section, we only consider isotropy contractions such that the initial ambient Lie algebra is semisimple and the subalgebra is reductive. Let $\mathfrak{k}=\mathfrak{h} \ltimes \mathfrak{m}$ be an isotropy contraction of a semisimple Lie algebra $\mathfrak{g}$. For $\mathfrak{g}$, one has equalities

$$
\operatorname{rk} \mathfrak{g}=\operatorname{ind} \mathfrak{g}=\operatorname{dim} \mathfrak{g} / / G \text {. }
$$

The first natural question is:

To which extent this remains true for isotropy contractions?

Recall that the complexity of a homogeneous space $G / H$, denoted $c(G / H)$, equals $\operatorname{trdeg} \mathbb{k}(G / H)^{B}$, where $B$ is a Borel subgroup of $G$, and $G / H$ is said to be spherical if $c(G / H)=0$. We refer to [28] for basic facts on complexity.

\subsection{Proposition.}

(1) We have ind $\mathfrak{k}=$ ind $\mathfrak{g}+2 c(G / H)$. In particular, ind $\mathfrak{k}=$ ind $\mathfrak{g}$ if and only if $H$ is a spherical subgroup of $G$.

(2) $\operatorname{dim} \mathfrak{k} / / K=\operatorname{dim} \mathfrak{z}_{\mathfrak{g}}(x)$, where $x \in \mathfrak{h}$ is an $\mathfrak{h}$-regular semisimple element.

Proof. (1) By [32], ind $\mathfrak{k}=\operatorname{trdeg} \mathbb{k}\left(\mathfrak{m}^{*}\right)^{H}+\operatorname{ind} \mathfrak{s}$, where $\mathfrak{s}$ is a generic stabiliser for $\left(H: \mathfrak{m}^{*}\right)$. Since $\mathfrak{m}$ is an orthogonal $\mathfrak{h}$-module, there is no difference between $\mathfrak{m}$ and $\mathfrak{m}^{*}$, the action $(H: \mathfrak{m})$ is stable [23] and therefore $\mathfrak{s}$ is reductive. Hence ind $\mathfrak{k}=\operatorname{dim} \mathfrak{m} / / H+\operatorname{rks}$. On the other hand, there is a formula for $c(G / H)$ in terms of the isotropy representation $(H: \mathfrak{m})$. Namely, $2 c(G / H)=$ $\operatorname{dim} \mathfrak{m} / / H-\operatorname{rk} \mathfrak{g}+\operatorname{rk} \mathfrak{s}$ [28, Cor. 2.2.9]. Hence the conclusion.

(2) By Theorem 6.2, $\operatorname{dim} \mathfrak{k} / / K=\operatorname{rkh}+\operatorname{dim} \mathfrak{m}^{\mathfrak{t}_{\mathfrak{h}}}$. The latter equals $\operatorname{dim} \mathfrak{z}_{\mathfrak{h}}(x)+\operatorname{dim} \mathfrak{m}^{x}$ for a regular semisimple element $x \in \mathfrak{t}_{\mathfrak{h}} \subset \mathfrak{h}$.

Remark. It is a general fact that the index of a Lie algebra cannot decrease under contraction. The previous result gives a precise meaning for this in case of isotropy contractions.

9.4 Corollary. If $\mathfrak{g}=\mathfrak{g}_{0} \oplus \mathfrak{g}_{1}$ is a $\mathbb{Z}_{2}$-grading and $\mathfrak{k}=\mathfrak{g}_{0} \ltimes \mathfrak{g}_{1}$ is the respective $\mathbb{Z}_{2}$-contraction, then ind $\mathfrak{k}=\operatorname{dim} \mathfrak{k} / / K=\operatorname{rk} \mathfrak{g}$.

Proof. As is well known, any symmetric subgroup $G_{0} \subset G$ is spherical, and $\mathfrak{g}_{0}$ contains a regular semisimple element of $\mathfrak{g}$. 
Thus, for $\mathbb{Z}_{2}$-contractions one obtains two, usually different, decompositions of the rank of $\mathfrak{g}$ :

$$
\mathrm{rk} \mathfrak{g}=\left\{\begin{array}{l}
\operatorname{ind} \mathfrak{k}=\mathrm{rk} \mathfrak{s}+\operatorname{dim} \mathfrak{g}_{1} / / G_{0} \\
\operatorname{dim} \mathfrak{k} / / K=\operatorname{rk} \mathfrak{g}_{0}+\operatorname{dim}\left(\mathfrak{g}_{1}\right)^{\mathfrak{t}_{0}}
\end{array}\right.
$$

where $\mathfrak{t}_{0}$ is a Cartan subalgebra of $\mathfrak{g}_{0}$.

If $\mathfrak{h}$ contains a $\mathfrak{g}$-regular semisimple element, then $\mathbb{k}[\mathfrak{g}]^{G}$ and $\mathbb{k}[\mathfrak{k}]^{K}$ are graded polynomial algebras of the same Krull dimension. The second natural question is:

Is there a relationship between the degrees of free homogeneous generators (basic invariants)?

Let $\operatorname{Deg}(A)$ denote the multiset of degrees of free generators of a graded polynomial algebra $A$. The elements of $\operatorname{Deg}(A)$ are assumed to be increasingly ordered.

9.5 Theorem. (1) If $\mathfrak{h}$ contains a $\mathfrak{g}$-regular semisimple element, then $\operatorname{Deg}\left(\mathbb{k}[\mathfrak{k}]^{K}\right) \leqslant \operatorname{Deg}\left(\mathbb{k}[\mathfrak{g}]^{G}\right)$ (componentwise inequalities).

(2) Suppose a regular nilpotent element of $\mathfrak{h}$ is also regular in $\mathfrak{g}$. Then $g$-exp $p_{\mathfrak{g}}(\mathfrak{g})=g-\exp _{\mathfrak{h}}(\mathfrak{h}) \uplus g-\exp _{\mathfrak{h}}(\mathfrak{m})$ (the union of multisets). Equivalently, $\operatorname{Deg}\left(\mathbb{k}[\mathfrak{k}]^{K}\right)=\operatorname{Deg}\left(\mathbb{k}[\mathfrak{g}]^{G}\right)$.

Proof. (1) Recall that $\mathfrak{k}=\lim _{t \rightarrow 0} \mathfrak{g}_{(t)}$. It is easily seen that this contraction gives rise to "a curve in the space of algebras of invariants" and to an embedding $\lim _{t \rightarrow 0} \mathbb{k}\left[\mathfrak{g}_{(t)}\right]^{G_{(t)}} \subset \mathbb{k}[\mathfrak{k}]^{K}$. The limit exists, because $\mathbb{k}\left[\mathfrak{g}_{(t)}\right]^{G_{(t)}}$ is graded and the (finite) dimension the homogeneous component of a given degree does not depend on $t$; so that the limit is taken in a suitable Grassmannian.

(2) Let $\{e, h, f\}$ be a principal $\mathfrak{s l}_{2}$-triple in $\mathfrak{h}$ (see $[45$, Ch. $6, \S 2.3]$ ). By the assumption, it is also a principal $\mathfrak{s l}_{2}$-triple in $\mathfrak{g}$. By a result of R. Brylinski [8], the generalised exponents of a $G$-module $V$ are obtained as follows. Take the subspace $V^{T}$ and its "e-limit" $\lim _{e}\left(V^{T}\right) \subset V$, see $[8, \S 2]$ for the precise definition. Then $g$-exp $(V)$ is the multiset of $h$-eigenvalues on $\lim _{e}\left(V^{T}\right)$. It is important that this "e-limit" depends only on the $\{e, h\}$-module structure on $V$. In our setting, $\mathfrak{g}$ and $\mathfrak{k}$ are isomorphic as $\mathfrak{h}$-modules, and $\mathfrak{k}=\mathfrak{h} \oplus \mathfrak{m}$ as $\mathfrak{h}$-module. Therefore

$$
g-\exp _{\mathfrak{g}}(\mathfrak{g})=g-\exp _{\mathfrak{h}}(\mathfrak{g})=g-\exp _{\mathfrak{h}}(\mathfrak{k})=g-\exp _{\mathfrak{h}}(\mathfrak{h}) \uplus g-\exp _{\mathfrak{h}}(\mathfrak{m}) .
$$

The second assertion follows from Theorem 6.2, because $\operatorname{Deg}\left(\mathbb{k}[\mathfrak{k}]^{K}\right)=$ $g$-exp $\operatorname{exh}_{\mathfrak{h}}(\mathfrak{k})+1$ (componentwise) and likewise for $\mathbb{k}[\mathfrak{g}]^{G}$. 
Part (2) of this theorem can be used for finding generalised exponents of certain representations.

9.6 Example. Let $\mathfrak{g}$ be $\mathfrak{s o}_{8}$ and $\mathfrak{h}$ the exceptional Lie algebra of type $\mathbf{G}_{2}(\operatorname{dim} \mathfrak{g}=28, \operatorname{dim} \mathfrak{h}=14)$. The restriction of the defining representation of $\mathfrak{g}$ to $\mathfrak{h}$ is the sum of $V(7)$, the 7 -dimensional simple $\mathfrak{h}$-module, and a 1 dimensional trivial module. Let $e \in \mathfrak{h}$ be a regular nilpotent element. It is known that $V(7)$ is a cyclic $e$-module. Therefore, as element of $\mathfrak{s o}_{8}, e$ has the Jordan form with blocks of size 7 and 1 . Hence $e$ is also regular in $\mathfrak{s o}_{8}$. Here $\mathfrak{m}=V(7) \oplus V(7)$. Since $g$-exp $\operatorname{exg}_{\mathfrak{g}}(\mathfrak{g})=\{1,3,3,5\}$ and $g$-exp $p_{\mathfrak{h}}(\mathfrak{h})=\{1,5\}$, we conclude that $g$ - $\exp _{\mathfrak{h}}(V(7))=\{3\}$. That is, the $\mathbb{k}[\mathfrak{h}]^{H}$-module $\operatorname{Mor}_{\mathfrak{h}}(\mathfrak{h}, V(7))$ is generated by the covariant of degree 3 .

This is also an instructive illustration to Theorem 8.1 and Corollary 8.2. Here $m=\mathrm{rk} \mathfrak{g}-\mathrm{rk} \mathfrak{h}=2$, hence $\pi_{K}$ is equidimensional. The basic covariant in $\operatorname{Mor}_{\mathfrak{h}}(\mathfrak{h}, V(7))$ vanishes on the subregular nilpotent orbit in $\mathcal{N}(\mathfrak{h})$. This follows from a result of Broer on the ideal defining the closure of the subregular nilpotent orbit $[7, \S 4]$. Therefore $\operatorname{codim}_{\mathcal{N}(\mathfrak{h})} \mathfrak{X}_{0}(\mathcal{N}(\mathfrak{h}))=2$ and $\mathcal{N}(\mathfrak{k})$ appears to be reducible.

From now on, we assume that $\mathfrak{k}$ is a $\mathbb{Z}_{2}$-contraction of $\mathfrak{g}$.

9.7 Theorem. Let $\mathfrak{g}=\mathfrak{g}_{0} \oplus \mathfrak{g}_{1}$ be a $\mathbb{Z}_{2}$-graded semisimple Lie algebra and $\mathfrak{k}=\mathfrak{g}_{0} \ltimes \mathfrak{g}_{1}$ its $\mathbb{Z}_{2}$-contraction. Then $\mathcal{N}(\mathfrak{k})$ is irreducible.

Proof. Let $\vartheta$ be the involution of $\mathfrak{g}$ determining the $\mathbb{Z}_{2}$-grading. It suffices to handle the case in which $\mathfrak{g}$ is not a sum of $\vartheta$-stable ideals. This means that either $\mathfrak{g}$ is simple or $\mathfrak{g}=\mathfrak{s} \dot{+} \mathfrak{s}$, where $\mathfrak{s}$ is simple and $\vartheta$ permutes the factors. In the second case, $\mathfrak{k}=\mathfrak{s} \ltimes \mathfrak{s}$ is a Takiff Lie algebra, and the required result is proved in [16, Theorem 2.4]. Therefore we concentrate on the first case.

From now on, $\mathfrak{g}$ is simple. Write $\mathcal{N}_{0}$ for the null-cone in $\mathfrak{g}_{0}$ and $K$ for the Takiff group $G_{0} \ltimes \mathfrak{g}_{1}$. Since $\mathfrak{g}_{1}$ is an orthogonal $G_{0}$-module, we do not distinguish $\mathfrak{g}_{1}$ and $\left(\mathfrak{g}_{1}\right)^{*}$.

1) Suppose $\vartheta$ is inner. Then $\mathrm{rk} \mathfrak{g}=\operatorname{rk} \mathfrak{g}_{0}$ and therefore the $\mathfrak{g}_{0}$-module $\mathfrak{g}_{1}$ has no zero weight space. As is noted in $\S 8$, the null-cone $\mathcal{N}(\mathfrak{k})$ is then isomorphic to $\mathcal{N}_{0} \times \mathfrak{g}_{1}$.

2) Suppose $\vartheta$ is outer. This is the difficult part of the proof, which relies on the classification of the involutions of simple Lie algebras. Recall that $m=$ $\operatorname{dim}\left(\mathfrak{g}_{1}\right)^{\mathfrak{t}_{0}}=\mathrm{rk} \mathfrak{g}-\mathrm{rk} \mathfrak{g}_{0}$.

$\left(a_{1}\right) \quad \mathrm{rkg}_{0}=\mathrm{rk} \mathfrak{g}-1$ and $m=1$. Here the assertion follows from Corollary 8.2. This happens if $\mathfrak{g}=\mathfrak{s o}_{2 n}$ and $\mathfrak{g}_{0}=\mathfrak{s o}_{2 k+1} \times \mathfrak{s o}_{2 l+1}$ with $k+l=n-1$. 
$\left(a_{2}\right) \quad r k \mathfrak{g}_{0}=\mathrm{rk} \mathfrak{g}-2$ and $m=2$. By Corollary $8.2, \pi_{K}$ is equidimensional. Still, $\mathcal{N}(\mathfrak{k})$ can be reducible a priori. To prove that this is not the case, consider the hierarchy $\mathfrak{X}_{0}\left(\mathcal{N}_{0}\right) \subset \overline{\mathfrak{X}_{1}\left(\mathcal{N}_{0}\right)} \subset \overline{\mathfrak{X}_{2}\left(\mathcal{N}_{0}\right)}=\mathcal{N}_{0}$ determined by the basic covariants of type $\mathfrak{g}_{1}$. Invoking the criterion of irreducibility (Theorem 8.1(iii)) with $m=2$ shows that only the condition with $i=0$ has to be satisfied. That is, we must have $\operatorname{codim}_{\mathcal{N}_{0}} \mathfrak{X}_{0}\left(\mathcal{N}_{0}\right) \geqslant 3$. This means that each nilpotent orbit in $\mathcal{N}_{0}$ of codimension 2 does not belong to $\mathfrak{X}_{0}\left(\mathcal{N}_{0}\right)$, i.e., there should exist a covariant $F \in \operatorname{Mor}_{G_{0}}\left(\mathfrak{g}_{0}, \mathfrak{g}_{1}\right)$ that does not vanish on such an orbit.

There are two involutions with $m=2$ in the exceptional algebras. In both cases, $\mathfrak{g}$ is of type $\mathbf{E}_{6}$ and $\mathfrak{g}_{0}$ is either $\mathbf{F}_{4}$ or $\mathbf{C}_{4}$. Furthermore, the degrees of basic covariants of type $\mathfrak{g}_{1}$ are 4,8 in both cases. Since $\mathfrak{g}_{0}$ is simple here, $\mathcal{N}_{0}$ has a unique orbit of codimension 2, the so-called subregular nilpotent orbit $\mathcal{O}_{\text {sub }}$. The closure of $\mathcal{O}_{\text {sub }}$ is normal and the equations of $\overline{\mathcal{O}}_{\text {sub }}$ in $\mathbb{k}\left[\mathcal{N}_{0}\right]$ are explicitly described, see $[7, \S 4]$. Therefore, it is not hard to verify that the covariant of degree 4 survives on $\mathcal{O}_{\text {sub }}$.

$\left(a_{3}\right)$ It remains to handle two series of $\left(\mathfrak{g}, \mathfrak{g}_{0}\right):\left(\mathfrak{s l}_{2 n}, \mathfrak{s p}_{2 n}\right)$ and $\left(\mathfrak{s l}_{n}, \mathfrak{s o}_{n}\right)$. In these cases, we explicitly describe the covariants of type $\mathfrak{g}_{1}$ and verify that the condition of Theorem 8.1(iii) is satisfied. Actually, we show that, for all $\mathbb{Z}_{2}$-contractions of simple Lie algebras, a stronger inequality holds, see Eq. (9.9) below.

Let us adapt Theorem 8.1 to our setting. We consider the stratification of $\mathcal{N}_{0}$ determined by covariants of type $\mathfrak{g}_{1}$. Since $\mathcal{N}_{0}$ consists of finitely many $G_{0}$-orbits, condition 8.1(iii) can be verified for each orbit separately. Therefore, it can be written as

$$
\operatorname{dim} \mathfrak{z}_{\mathfrak{g}_{0}}(x)-\operatorname{rk} \mathfrak{g}_{0}>m-\operatorname{dim} \operatorname{span}\left\{F_{1}(x), \ldots, F_{m}(x)\right\} \text { if } x \in \mathcal{N}_{0} \backslash \mathfrak{X}_{m}\left(\mathcal{N}_{0}\right),
$$

cf. Remark 8.3(1). What we are going to prove is:

$$
\operatorname{dim} \mathfrak{z}_{\mathfrak{g}_{0}}(x)-\mathrm{rk} \mathfrak{g}_{0} \geqslant 2\left(m-\operatorname{dim} \operatorname{span}\left\{F_{1}(x), \ldots, F_{m}(x)\right\}\right) \text { for any } x \in \mathcal{N}_{0} .
$$

Clearly, the last version is stronger and has an advantage of being stated more uniformly.

9.10 Theorem. Inequality (9.9) holds for any $\mathbb{Z}_{2}$-grading of a simple Lie algebra $\mathfrak{g}$.

Proof. Since the difference in the left-hand side of (9.9) is always even, there is no distinction between inequalities (9.8) and (9.9) for $m \leqslant 2$. Therefore 
the proof of Theorem 9.7 shows that it remains to verify Eq. (9.9) for the following series of $\mathbb{Z}_{2}$-gradings:

- $\mathfrak{g}_{0}=\mathfrak{s p}(V), \mathfrak{g}_{1}=\wedge_{0}^{2}(V), \operatorname{dim} V=2 n$.

- $\mathfrak{g}_{0}=\mathfrak{s o}(V), \mathfrak{g}_{1}=\mathcal{S}_{0}^{2}(V)$. Here one actually has two series, depending on the parity of $\operatorname{dim} V$.

We use familiar matrix models of classical Lie algebras and their representations. In the following computations, we need the fact that the nilpotent $G_{0}$-orbits are classified by certain partitions of $\operatorname{dim} V$, see [39, IV.2.15], [45, Ch. 6 §2.2]. A minor unpleasant phenomenon related to $\mathfrak{s o}_{2 n}$ is that there are two isomorphic $\mathrm{SO}_{2 n}$-orbits corresponding to a "very even partition". This does not affect, however, our computations. For $x \in \mathcal{N}_{0}$, let $\boldsymbol{\eta}=\left(\eta_{1}, \eta_{2}, \ldots\right)$ denote the corresponding partition. Write $\left(\hat{\eta}_{1}, \hat{\eta}_{2}, \ldots, \hat{\eta}_{s}\right)$ for the dual partition. This means in particular that $s=\eta_{1}$. What we need from these partitions is an explicit formula for $\operatorname{dim} \mathfrak{z}_{\mathfrak{g}_{0}}(x)$ and a way to determine $i$ such that $x \in \mathfrak{X}_{i}\left(\mathcal{N}_{0}\right)$.

Let us begin with the symplectic case. Let $J$ be a skew-symmetric nondegenerate bilinear form on $V$, which is identified with its matrix in a certain basis for $V$. Then

$-\mathfrak{s p}_{2 n}=\mathfrak{s p}($
$x J$ is symmetric $\}$;

- the representation space $\wedge_{0}^{2}(V)$ can be regarded as the space of skewsymmetric matrices modulo one-dimensional subspace generated by $J$. The $\mathfrak{s p}_{2 n}$-action on the space of skew-symmetric matrices is given by $(x, A) \mapsto x J A+$ $A(x J)^{t}$.

In this case $m=n-1$, i.e., there are $n-1$ basic covariants of type $\mathfrak{g}_{1}$. Since any regular nilpotent element in $\mathfrak{s p}_{2 n}$ is also regular in $\mathfrak{s l}_{2 n}$, the generalised exponents of the $\mathfrak{g}_{0}$-module $\mathfrak{g}_{1}$ can be found using Theorem 9.5(2). These are $2,4, \ldots, 2 n-2$. The key observation is that the corresponding covariants have a very simple expression. Namely, consider the maps $\left(x \in \mathfrak{s p}_{2 n}\right) \mapsto F_{i}(x)=x^{2 i} J$, $i=1, \ldots, n-1$. It is easily seen that $x^{2 i} J$ is skew-symmetric and each $F_{i}$ is $S p_{2 n}$-equivariant. Because the $F_{i}$ 's are linearly independent over $\mathbb{k}\left[\mathfrak{g}_{0}\right]^{G_{0}}$, these are precisely the basic covariants.

9.11 Proposition. Inequality (9.9) is satisfied for $\left(S p(V), \wedge_{0}^{2}(V)\right)$.

Proof. By [17, Corollary 3.8(a)], the dimension of the centraliser of $x$ in $\mathfrak{g}_{0}=\mathfrak{s p}_{2 n}$ is given by the formula $\operatorname{dim} \mathfrak{z}_{\mathfrak{g}_{0}}(x)=\frac{1}{2}\left(\sum_{i} \hat{\eta}_{i}^{2}+\#\left\{j \mid \eta_{j}\right.\right.$ is odd $\left.\}\right)$. 
The maximal nonzero power of $x$ is determined by the size of the maximal Jordan block, i.e., $\eta_{1}$. Therefore $x \in \mathfrak{X}_{i}\left(\mathcal{N}_{0}\right)$ if and only if $x^{2 i} \neq 0$ and $x^{2 i+2}=0$ if and only if $\left[\frac{\eta_{1}-1}{2}\right]=i$. Hence inequality (9.9), which we wish to prove, can be written as

$$
2\left[\frac{\eta_{1}-1}{2}\right]+\frac{1}{2}\left(\sum_{i=1}^{s} \hat{\eta}_{i}^{2}+\#\left\{j \mid \eta_{j} \text { is odd }\right\}\right)-n-2(n-1) \geqslant 0 .
$$

Using the relations $\sum \hat{\eta}_{i}=2 n$ and $\eta_{1}=s$, the left-hand side is transformed as follows:

$$
\begin{aligned}
& 2\left[\frac{\eta_{1}-1}{2}\right]+ \frac{1}{2}\left(\sum_{i=1}^{s} \hat{\eta}_{i}^{2}+\#\left\{j \mid \eta_{j} \text { is odd }\right\}\right)-\frac{3}{2} \sum \hat{\eta}_{i}+2= \\
& 2\left[\frac{s-1}{2}\right]+\frac{1}{2}\left(\sum_{i=1}^{s}\left(\hat{\eta}_{i}^{2}-3 \hat{\eta}_{i}\right)+\#\left\{j \mid \eta_{j} \text { is odd }\right\}\right)+2= \\
& \frac{1}{2}\left(\sum_{i=1}^{s}\left(\hat{\eta}_{i}-1\right)\left(\hat{\eta}_{i}-2\right)+\#\left\{j \mid \eta_{j} \text { is odd }\right\}\right)+2\left[\frac{s+1}{2}\right]-s .
\end{aligned}
$$

The first group of summands is non-negative, and so is the last group. Thus, inequality (9.9) holds for any nilpotent orbit in $\mathfrak{s p}_{2 n}$.

We continue with the orthogonal case, with $\operatorname{dim} V=N$. Here $\mathfrak{g}_{0}$ is the space of skew-symmetric $N \times N$-matrices and $\mathfrak{g}_{1}=\mathcal{S}_{0}^{2}(V)$ is the space of traceless symmetric $N \times N$-matrices.

If $N=2 n+1$, then $m=n$. In this case, a regular nilpotent element of $\mathfrak{s o}_{2 n+1}$ is also regular in $\mathfrak{s l}_{2 n+1}$, so that Theorem 9.5(2) applies, and $g$-exp $p_{G_{0}}\left(\mathfrak{g}_{1}\right)=$ $\{2,4, \ldots, 2 n\}$. Similarly to the symplectic case, we find that $x \mapsto F_{i}(x)=x^{2 i}$, $i=1,2, \ldots, n$, are the basic covariants. If $N=2 n$, then $m=n-1$. A regular nilpotent element of $\mathfrak{s o}_{2 n}$ is not regular in $\mathfrak{s l}_{2 n}$, but $F_{1}, \ldots, F_{n-1}$ are still the basic covariants. For, the $F_{i}$ 's are linearly independent over $\mathbb{k}\left[\mathfrak{g}_{1}\right]^{G_{0}}$ and neither of them vanishes on the regular nilpotent orbit in $\mathfrak{s o}_{2 n}$.

9.12 Proposition. Inequality (9.9) is satisfied for $\left(S O(V), \mathcal{S}_{0}^{2}(V)\right)$.

Proof. By [17, Corollary 3.8(a)], the dimension of the centraliser of $x$ in $\mathfrak{g}_{0}=\mathfrak{s o}_{N}$ is given by the formula $\operatorname{dim} \mathfrak{z}_{\mathfrak{g}_{0}}(x)=\frac{1}{2}\left(\sum_{i} \hat{\eta}_{i}^{2}-\#\left\{j \mid \eta_{j}\right.\right.$ is odd $\left.\}\right)$. The constraints imposed on partitions in the orthogonal case imply that $\hat{\eta}_{1} \equiv$ $\operatorname{dim} V(\bmod 2)$.

The maximal nonzero power of $x$ is determined by the size of the maximal 
Jordan block. Therefore $x \in \mathfrak{X}_{i}\left(\mathcal{N}_{0}\right)$ if and only if $x^{2 i} \neq 0$ and $x^{2 i+2}=0$ if and only if $\left[\frac{\eta_{1}-1}{2}\right]=i$. The following computations are slightly different for $\mathfrak{s o}_{2 n+1}$ and $\mathfrak{s o}_{2 n}$.

1. $N=2 n+1$. Here inequality (9.9) can be written as

$$
2\left[\frac{\eta_{1}-1}{2}\right]+\frac{1}{2}\left(\sum_{i} \hat{\eta}_{i}^{2}-\#\left\{j \mid \eta_{j} \text { is odd }\right\}\right)-3 n \geqslant 0 .
$$

Using the relations $\sum \hat{\eta}_{i}=2 n+1$ and $\eta_{1}=s$, the left-hand side is transformed as follows:

$$
\begin{aligned}
& 2\left[\frac{s-1}{2}\right]+\frac{1}{2}\left(\sum_{i=1}^{s} \hat{\eta}_{i}^{2}-\#\left\{j \mid \eta_{j} \text { is odd }\right\}\right)-\frac{3}{2}\left(\sum_{i=1}^{s} \hat{\eta}_{i}-1\right)= \\
& 2\left[\frac{s-1}{2}\right]+\frac{1}{2}\left(\sum_{i=1}^{s}\left(\hat{\eta}_{i}^{2}-3 \hat{\eta}_{i}+2\right)-2 s+3-\#\left\{j \mid \eta_{j} \text { is odd }\right\}\right)= \\
& \quad \frac{1}{2}\left(\sum_{i=1}^{s}\left(\hat{\eta}_{i}-1\right)\left(\hat{\eta}_{i}-2\right)-\#\left\{j \mid \eta_{j} \text { is odd }\right\}+4\left[\frac{s+1}{2}\right]-2 s-1\right)=: \mathrm{L} .
\end{aligned}
$$

To see that $L$ is nonnegative, consider several cases.

(a) $\hat{\eta}_{1}=1$ and hence all $\hat{\eta}_{i}=1$. Then $s=2 n+1$ and $\mathrm{L}=0$.

(b) $\hat{\eta}_{1}=3$ and therefore $\boldsymbol{\eta}=\left(\eta_{1}, \eta_{2}, \eta_{3}\right)$. Then $\sum_{i=1}^{s}\left(\hat{\eta}_{i}-1\right)\left(\hat{\eta}_{i}-2\right)=2 \eta_{3}$.

Hence

$$
\mathrm{L}=\eta_{3}+2\left[\frac{\eta_{1}+1}{2}\right]-\eta_{1}-\frac{1}{2}\left(1+\#\left\{j \mid \eta_{j} \text { is odd }\right\}\right) .
$$

Taking into account that the even parts in $\left(\eta_{1}, \eta_{2}, \eta_{3}\right)$ occur pairwise and $\eta_{1}+$ $\eta_{2}+\eta_{3}$ is odd, one quickly verifies that $\mathrm{L}$ is always nonnegative.

(c) $\hat{\eta}_{1} \geqslant 5$. Then $\sum_{i=1}^{s}\left(\hat{\eta}_{i}-1\right)\left(\hat{\eta}_{i}-2\right) \geqslant \hat{\eta}_{1}+2 \geqslant \#\left\{j \mid \eta_{j}\right.$ is odd $\}+2$. Next, $4\left[\frac{s+1}{2}\right]-2 s-1 \geqslant-1$. Hence $L$ is positive.

Thus, inequality (9.9) holds for any nilpotent orbit in $\mathfrak{s o}_{2 n+1}$.

2. $N=2 n$. Here the inequality we need to prove reads

$$
2\left[\frac{\eta_{1}-1}{2}\right]+\frac{1}{2}\left(\sum_{i} \hat{\eta}_{i}^{2}-\#\left\{j \mid \eta_{j} \text { is odd }\right\}\right)-n-2(n-1) \geqslant 0 .
$$

Using the relations $\sum \hat{\eta}_{i}=2 n$ and $\eta_{1}=s$, the left-hand side is being transformed to

$$
\frac{1}{2}\left(\sum_{i=1}^{s}\left(\hat{\eta}_{i}-1\right)\left(\hat{\eta}_{i}-2\right)-\#\left\{j \mid \eta_{j} \text { is odd }\right\}\right)+2\left[\frac{s+1}{2}\right]-s=: \mathrm{L} .
$$

Again, consider some cases. 
(a) $\hat{\eta}_{1}=2$, i.e., $x$ has only two Jordan blocks $\left(\eta_{1}, \eta_{2}\right)$. Then $\eta_{1}, \eta_{2}$ have the same parity, and in both cases $\mathrm{L}=0$.

(b) $\hat{\eta}_{1} \geqslant 4$. Then $\sum_{i=1}^{s}\left(\hat{\eta}_{i}-1\right)\left(\hat{\eta}_{i}-2\right) \geqslant \hat{\eta}_{1}+2>\#\left\{j \mid \eta_{j}\right.$ is odd $\}$. Since $2\left[\frac{s+1}{2}\right]-s \geqslant 0$, the total expression is positive.

Thus, inequality (9.9) holds for any nilpotent orbit in $\mathfrak{s o}_{2 n}$.

This completes the proof of Theorem 9.10.

Thus, all verifications needed to complete the proof of Theorem 9.7 are done. Below, we gather our results on $\mathbb{Z}_{2}$-contractions of semisimple Lie algebras.

9.13 Theorem. Let $\mathfrak{k}=\mathfrak{g}_{0} \ltimes \mathfrak{g}_{1}$ be a $\mathbb{Z}_{2}$-contraction of a semisimple Lie algebra $\mathfrak{g}$. Then

(1) $\mathbb{k}[\mathfrak{k}]^{K}$ is a polynomial algebra of Krull dimension $\mathrm{rk} \mathfrak{g}$;

(2) $\mathcal{N}(\mathfrak{k})$ is an irreducible complete intersection. If $\mathbb{k}[\mathfrak{k}]^{K}=\mathbb{k}\left[f_{1}, \ldots, f_{l}\right], l=$ $\mathrm{rk} \mathfrak{g}$, then the ideal of $\mathcal{N}(\mathfrak{k})$ in $\mathbb{k}[\mathfrak{k}]$ is generated by $f_{1}, \ldots, f_{l}$.

(3) the quotient morphism $\pi_{K}: \mathfrak{k} \rightarrow \mathfrak{k} / / K$ is equidimensional;

(4) $\mathbb{k}[\mathfrak{k}]$ is a free $\mathbb{k}[\mathfrak{k}]^{K}$-module.

(5) if $\kappa: \mathfrak{g}_{0} \oplus \mathfrak{g}_{1} \rightarrow \mathfrak{g}_{0} \oplus \mathfrak{g}_{1}$ is defined by $\kappa\left(x_{0}, x_{1}\right)=\left(x_{0},\left[x_{0}, x_{1}\right]\right)$, then $\overline{\operatorname{Im} \kappa}=\mathcal{N}^{u}(\mathfrak{q})$ and it is a factorial complete intersection of codimension $\operatorname{rk} \mathfrak{g}-\operatorname{rk} \mathfrak{g}_{0}$.

(6) the coadjoint representation of $\mathfrak{k}$ has a generic stabiliser.

Proof. Part (1) follows from Theorem 6.2. The irreducibility in Part (2) is just Theorem 9.7. Let $x \in \mathcal{N}_{0}$ be a regular nilpotent element. Then $\tilde{x}=(x, 0) \in$ $\mathcal{N}(\mathfrak{k})$, and the description of basic invariants $f_{1}, \ldots, f_{l}$ in Theorem 6.2 shows that $\left(d f_{1}\right)_{\tilde{x}}, \ldots,\left(d f_{l}\right)_{\tilde{x}}$ are linearly independent. Then a standard argument shows that the ideal of $\mathcal{N}(\mathfrak{k})$ is generated by $f_{1}, \ldots, f_{l}$ (cf. [21, Prop. 6].) Part (3) follows from (2) and Theorem 8.1(2). Part (4) is a formal consequence of Parts (1) and (3). Part (5) follows from Theorem 8.8 and the irreducibility of $\mathcal{N}(\mathfrak{k})$. Since the isotropy representation of any symmetric subalgebra of $\mathfrak{g}$ is polar, part (6) follows from Theorem 5.8.

To prove the irreducibility of $\mathcal{N}(\mathfrak{k})$, inequality (9.8) is sufficient. However, our efforts in proving stronger inequality (9.9) are not in vain, because that result also has a geometric meaning. 
9.14 Theorem. Let $\mathfrak{g}=\mathfrak{g}_{0} \oplus \mathfrak{g}_{1}$ be a $\mathbb{Z}_{2}$-grading. Consider the semidirect product $\tilde{\mathfrak{k}}=\mathfrak{g}_{0} \ltimes\left(\mathfrak{g}_{1} \oplus \mathfrak{g}_{1}\right)$ and the corresponding adjoint representation $(\tilde{K}: \tilde{\mathfrak{k}})$. Then the quotient morphism $\pi_{\tilde{K}}$ is equidimensional.

Proof. The criterion for equidimensionality of $\pi_{\tilde{K}}$, Theorem 8.1(i), written out in this case yields precisely inequality (9.9).

Main efforts in this section were devoted to $\mathbb{Z}_{2}$-contractions of $\mathfrak{g}$. However, there are interesting examples of other isotropy contractions with full bunch of good properties.

9.15 Examples. 1. Suppose $\mathfrak{g}=\mathfrak{s o}_{7}$ and $\mathfrak{h}$ is a simple subalgebra of type $\mathbf{G}_{2}$. It is a "truncation" of Example 9.6. Here $\mathfrak{m}=V(7)$, and one easily verifies that all conclusions of Theorem 9.13 hold for $\mathfrak{k}=\mathfrak{h} \ltimes \mathfrak{m}$.

2. $\mathfrak{g}=\mathfrak{s l}_{2 n+1}$ and $\mathfrak{h}=\mathfrak{s p}_{2 n}=\mathfrak{s p}(V)$. Here the $\mathfrak{s p}(V)$-module $\mathfrak{m}$ equals $\wedge^{2}(V) \oplus V \oplus V$. Since the $\mathfrak{s p}(V)$-module $V$ has no zero-weight space, the structure of $\mathcal{N}(\mathfrak{h} \ltimes \mathfrak{m})$ is essentially the same as for the $\mathbb{Z}_{2}$-contraction of the symmetric pair $\left(\mathfrak{s l}_{2 n}, \mathfrak{s p}_{2 n}\right)$.

Remark. Our proofs of Theorems 9.7 and 9.10 use classification of involutory automorphisms and explicit considerations of cases. It would be extremely interesting to find a case-free proof for the irreducibility of $\mathcal{N}(\mathfrak{k})$. Especially, because the corresponding irreducibility result for the Takiff algebra $\mathfrak{g} \ltimes \mathfrak{g}$ can be derived without checking cases. We discuss this topic in the following section.

\section{§10. Reductive Takiff Lie Algebras and their Representations}

The attentive reader may have noticed that we stated and proved the stronger inequality (9.9) only for the $\mathbb{Z}_{2}$-gradings of simple Lie algebras, leaving aside the permutation of two factors in $\mathfrak{g} \times \mathfrak{g}$ and the corresponding Takiff algebra $\hat{\mathfrak{g}}$.

The situation here is as follows. By Theorem 8.1, the counterpart of inequality (9.8) for $\hat{\mathfrak{g}}$ is equivalent to the irreducibility of $\mathcal{N}(\hat{\mathfrak{g}})$, and this was already proved by Geoffriau [16]. His proof consists of explicit verifications for all simple types. It was noticed by M. Brion [6] that a classification-free proof of (9.8) for $\hat{\mathfrak{g}}$, and hence the irreducibility of $\mathcal{N}(\hat{\mathfrak{g}})$, can be derived from the fact that $\mathcal{N}$ is a complete intersection having only rational singularities, see below. The advantage of the Takiff algebra case is that the rather mysterious term $\operatorname{dim} \operatorname{span}\left\{F_{1}(x), \ldots, F_{m}(x)\right\}$ is being interpreted as the rank of the differential of the quotient map $\pi_{G}: \mathfrak{g} \rightarrow \mathfrak{g} / / G$ at $x$. 
On the other hand, we will prove here the counterpart of (9.9) for $\hat{\mathfrak{g}}$, using the classification. Brion's idea cannot be applied directly to obtain a case-free proof of that stronger result. The reason for being interested in proving a counterpart of (9.9) for $\hat{\mathfrak{g}}$ is that we deduce from this the equidimensionality of some other quotient morphisms, see Theorems 10.8,10.9.

We work in the setting of case $\mathbf{B}$ ) from $\S 8$.

10.1 Definition. Let $\rho: G \rightarrow G L(V)$ be a representation of a connected reductive group $G$. Then $V$ or $\rho$ is said to be extremely good if

(1) $\mathbb{k}[V]^{G}$ is a polynomial algebra;

(2) $\max \operatorname{dim}_{x \in V} G \cdot x=\operatorname{dim} V-\operatorname{dim} V / / G$;

(3) If $\pi_{G}: V \rightarrow V / / G$ is the quotient morphism, then $\Omega:=\{x \in V \mid$ $\left(\mathrm{d} \pi_{G}\right)_{x}$ is onto 3 is a big open subset of $V$;

(4) $\mathcal{N}(V):=\pi_{G}^{-1}\left(\pi_{G}(0)\right)$ consists of finitely many $G$-orbits;

(5) $\mathcal{N}(V)$ is irreducible and has only rational singularities.

Note that properties (1)-(3) are those appearing in Theorem 7.1. Recall from $\S 7$ that if $G$ is semisimple, then (2) and (3) are always satisfied.

10.2 Theorem. Let $V$ be an extremely good $G$-module and $\hat{V}=V \ltimes V$ the corresponding $\hat{G}$-module. Then

(i) $\mathcal{N}^{\hat{G}}(\hat{V})=\mathcal{N}(\hat{V})$ is an irreducible complete intersection;

(ii) the ideal of $\mathcal{N}(\hat{V})$ in $\mathbb{k}[\hat{V}]$ is generated by the basic invariants in $\mathbb{k}[\hat{V}]^{\hat{G}}$;

(iii) $\pi_{\hat{G}}: \hat{V} \rightarrow \hat{V} / / \hat{G}$ is equidimensional and $\mathbb{k}[\hat{V}]$ is a free $\mathbb{k}[\hat{V}]^{\hat{G}}$-module.

Proof. Let $f_{1}, \ldots, f_{m}$ be algebraically independent generators of $\mathbb{k}[V]^{G}$. By Theorem $7.1, \mathbb{k}[\hat{V}]^{\hat{G}}$ is freely generated by the polynomials $f_{1}, \ldots, f_{m}$, $\widehat{F}_{f_{1}}, \ldots, \widehat{F}_{f_{m}}$. Recall from $\S 8$ the stratification of the null-cone:

$$
\mathfrak{Y}_{i}(\mathcal{N}(V))=\left\{v \in \mathcal{N}(V) \mid \operatorname{rk}\left(d \pi_{G}\right)_{v}=i\right\}, \quad i=0,1, \ldots, m .
$$

Since $\mathcal{N}(V)$ contains finitely many $G$-orbits, $\pi_{G}$ is equidimensional. If $G \cdot x$ is the dense $G$-orbit in $\mathcal{N}(V)$, then $\operatorname{dim} G \cdot x=\operatorname{dim} V-m$ and therefore $x \in \mathfrak{Y}_{m}(\mathcal{N}(V))$ [20, Korollar 2]. (Corollary 2 is stated in Knop's article under the assumption that $G$ is semisimple. However, that proof works also for reductive groups as long as conditions (2) and (3) are satisfied.) Since $\mathcal{N}(V)$ is irreducible and 
$\mathfrak{Y}_{m}(\mathcal{N}(V)) \neq \varnothing$, it is a complete intersection. The condition of the irreducibility of $\mathcal{N}(\hat{V})$ (Theorem 8.10(iii)) can be written as

$$
\operatorname{dim} V-\operatorname{dim} G \cdot v+\operatorname{rk}\left(d \pi_{G}\right)_{v}>2 m \quad \text { if } v \notin \mathfrak{Y}_{m}(\mathcal{N}(V)) .
$$

We derive this inequality from a property of the local ring of (the closure of) the orbit $G \cdot v \subset \mathcal{N}(V)$. Let $\mathcal{O}$ be this local ring. Then $\operatorname{dim} \mathcal{O}=\operatorname{dim} \mathcal{N}(V)-\operatorname{dim} G \cdot v$ and $\operatorname{edim} \mathcal{O}=\operatorname{dim} T_{v} \mathcal{N}(V)-\operatorname{dim} G \cdot v=\operatorname{dim} \mathfrak{g}_{v}-\operatorname{rk}\left(d \pi_{G}\right)_{v}$. Here edim $\mathcal{O}$ is the embedding dimension of $\mathcal{O}$ and $T_{v} \mathcal{N}(V)$ is the tangent space of $\mathcal{N}(V)$ at $v$. Since $\mathcal{N}(V)$ has only rational singularities, so has $\mathcal{O}$. By a result of Goto-Watanabe (see [25, Theorem 2']), if a local ring $\mathcal{O}$ is a complete intersection with only rational singularities and $\operatorname{dim} \mathcal{O}>0$, then $\operatorname{edim} \mathcal{O}<2 \operatorname{dim} \mathcal{O}$. Using the above expressions for $\operatorname{edim} \mathcal{O}$ and $\operatorname{dim} \mathcal{O}$, one obtains inequality (10.3), and thereby the irreducibility of $\mathcal{N}(\hat{V})$.

All other statements of the theorem are consequences of the fact that $\mathcal{N}(\hat{V})$ is irreducible. By Theorem 8.10(ii), $\pi_{\hat{G}}$ is equidimensional. If $v \in \mathfrak{Y}_{m}(\mathcal{N}(V))$, then the differentials of the generators $f_{1}, \ldots, f_{m}, \widehat{F}_{f_{1}}, \ldots, \widehat{F}_{f_{m}}$ are linearly independent at $(v, 0) \in \mathcal{N}(\hat{V}) \subset \hat{V}$. This fact and the irreducibility of $\mathcal{N}(\hat{V})$ imply that $\mathcal{N}(\hat{V})$ is a complete intersection whose ideal is generated by the polynomials $f_{1}, \ldots, f_{m}, \widehat{F}_{f_{1}}, \ldots, \widehat{F}_{f_{m}}$ (cf. [21, Prop. 6]).

Remark. The most subtle point in the definition of extremely good representations is the rationality of singularities of $\mathcal{N}(V)$. For the adjoint representations, this result is due to W. Hesselink [18]. The idea to exploit the fact that $\mathcal{N}=\mathcal{N}(\mathfrak{g})$ is a complete intersection with only rational singularities, and to use the Goto-Watanabe inequality for local rings is due to M. Brion [6]. Since $(G, \mathrm{Ad})$ is extremely good, this approach yields a conceptual proof of [16, Theorem 2.4].

10.4 Corollary. If $V$ is extremely good, then the closure of the image of the map

$$
\varkappa: V \times \mathfrak{g} \rightarrow V \times V, \quad(v, x) \mapsto(v, x \cdot v),
$$

is a factorial complete intersection of codimension $m=\operatorname{dim} V / / G$ and the ideal of $\overline{\operatorname{Im} \varkappa}$ is generated by $\widehat{F}_{f_{1}}, \ldots, \widehat{F}_{f_{m}}$.

Proof. This follows from the irreducibility of $\mathcal{N}(\hat{V})$ and Theorem 8.11.

Since conditions (4) and (5) are quite restrictive, there are only a few extremely good representations. Below is a list of such irreducible representations known to this author such that $G$ is simple and $\mathbb{k}[V]^{G} \neq \mathbb{k}$, except the adjoint ones: 


$$
\begin{aligned}
& \left(\mathbf{B}_{n} \text { or } \mathbf{D}_{n}, \varphi_{1}\right),\left(\mathbf{B}_{3}, \varphi_{3}\right),\left(\mathbf{B}_{4}, \varphi_{4}\right),\left(\mathbf{G}_{2}, \varphi_{1}\right),\left(\mathbf{A}_{n}, 2 \varphi_{1}\right),\left(\mathbf{A}_{2 n-1}, \varphi_{2}\right), \\
& \left(\mathbf{E}_{6}, \varphi_{1}\right),\left(\mathbf{C}_{3}, \varphi_{3}\right),\left(\mathbf{A}_{5}, \varphi_{3}\right),\left(\mathbf{D}_{6}, \varphi_{6}\right),\left(\mathbf{E}_{7}, \varphi_{1}\right),\left(\mathbf{B}_{5}, \varphi_{5}\right),\left(\mathbf{F}_{4}, \varphi_{1}\right),\left(\mathbf{C}_{n}, \varphi_{2}\right) .
\end{aligned}
$$

The representations are given by their highest weights, and $\left\{\varphi_{i}\right\}$ are fundamental weights of $G$ with numbering from [45]. For all representations in the list but the last one, the algebra of covariants, $\mathbb{k}[V]^{U}$, is polynomial [4] (here $U$ is a maximal unipotent subgroup of $G)$. Therefore the same is true for $\mathbb{k}[\mathcal{N}(V)]^{U}$. Then a result of Kraft (see $[3,1.5-6]$ ) shows that $\mathcal{N}(V)$ has rational singularities.

I conjecture that if $G$ is simple and $V$ is a simple $G$-module, then $V$ is extremely good if and only if $\operatorname{dim} V \leqslant \operatorname{dim} G$. Practically, this means that one has to only verify that $\mathcal{N}(V)$ has rational singularities for the following representations:

$\left(\mathbf{A}_{6}, \varphi_{3}\right),\left(\mathbf{A}_{7}, \varphi_{3}\right),\left(\mathbf{B}_{6}, \varphi_{6}\right),\left(\mathbf{D}_{7}, \varphi_{7}\right)$.

For $V=\mathfrak{g}$, inequality (10.3) reads

$$
\operatorname{dim} \mathfrak{z}_{\mathfrak{g}}(x)+\operatorname{rk}\left(d \pi_{G}\right)_{x}>2 \operatorname{rkg}=2 m \text { if } x \notin \mathfrak{X}_{m}(\mathcal{N})=\mathcal{N}^{r e g} .
$$

This inequality was proved in [16, 2.6-2.15] in a case-by-case fashion. Below, we prove a stronger result, which is the counterpart of inequality (9.9) in the context of Takiff algebras. By the Morozov-Jacobson theorem [45, Ch. 3, Theorem 1.3], any $x \in \mathcal{N} \backslash\{0\}$ can be embedded in an $\mathfrak{s l}_{2}$-triple $\{x, h, y\}$, where $h$ is semisimple; $x$ is said to be even if the ad $h$-eigenvalues in $\mathfrak{g}$ are even. Following E. B. Dynkin, $h$ is called a characteristic of $x$.

10.6 Theorem. Let $\mathfrak{g}$ be a simple Lie algebra and $x \in \mathcal{N}$. Then

$$
\mathrm{L}:=\operatorname{dim} \mathfrak{z}_{\mathfrak{g}}(x)+2 \operatorname{rk}\left(d \pi_{G}\right)_{x}-3 \operatorname{rk} \mathfrak{g} \geqslant 0 .
$$

If $\mathfrak{g}=\mathfrak{s l}_{n}$, then $\mathrm{L}=0$ if and only if the matrix $x$ has at most two Jordan blocks. Furthermore, if $\mathfrak{g} \neq \mathfrak{s l}_{2 n+1}$, then $\mathrm{L}=0$ if and only if $x$ is even and $\left[\mathfrak{z}_{\mathfrak{g}}(h), \mathfrak{z}_{\mathfrak{g}}(h)\right]$ is a sum of several copies of $\mathfrak{s l}_{2}$. (Here $h$ is a characteristic of $x$ ).

Proof. The proof is case-by-case. However, the computations themselves are much shorter and more transparent than those in [16], because our inequality is stronger, and we use formulae for $\operatorname{dim} \mathfrak{z}_{\mathfrak{g}}(x)$ in terms of dual partitions (already used for $S p$ and $S O$ in the proof of Propositions 9.11 and 9.12).

For the classical series, we work with the partition of $x$; while for the exceptional algebras the explicit classification of nilpotent orbits is used. If $\mathfrak{g}=$ $\mathfrak{g}(\mathbb{V})$ is classical and $x \in \mathfrak{g}(\mathbb{V})$ is nilpotent, then $\boldsymbol{\eta}=\left(\eta_{1}, \eta_{2}, \ldots\right)$ is the partition of $\operatorname{dim} \mathbb{V}$ corresponding to $x$ and $\left(\hat{\eta}_{1}, \ldots, \hat{\eta}_{s}\right)$ is the dual partition. Here $s=\eta_{1}$. For $S p$ and $S O$, our analysis is quite similar to those in Propositions 9.11 and 9.12 . 
(A) $\mathfrak{g}=\mathfrak{s l}(\mathbb{V}), \operatorname{dim} \mathbb{V}=n+1$.

Here $\operatorname{dim} \mathfrak{z}_{\mathfrak{g}}(x)=\sum_{i=1}^{s} \hat{\eta}_{i}^{2}-1$ and $\operatorname{rk}\left(d \pi_{G}\right)_{x}=\eta_{1}-1=s-1$ [35, Theorem 4.2.1]. Then

$\mathrm{L}=\sum_{i=1}^{s} \hat{\eta}_{i}^{2}-1+2(s-1)-3 n=\sum_{i=1}^{s} \hat{\eta}_{i}^{2}-3 \sum_{i=1}^{s} \hat{\eta}_{i}+2 s=\sum_{i=1}^{s}\left(\hat{\eta}_{i}-1\right)\left(\hat{\eta}_{i}-2\right) \geqslant 0$.

This expression equals zero if and only if all $\hat{\eta}_{i} \leqslant 2$, i.e., $x$ has at most two Jordan blocks.

(B) $\mathfrak{g}=\mathfrak{s o}(\mathbb{V}), \operatorname{dim} \mathbb{V}=2 n+1$.

Here $\hat{\eta}_{1}$ is odd, $\operatorname{dim} \mathfrak{z}_{\mathfrak{g}}(x)=\frac{1}{2}\left(\sum_{i} \hat{\eta}_{i}^{2}-\#\left\{j \mid \eta_{j}\right.\right.$ is odd $\left.\}\right)$, and $\operatorname{rk}\left(d \pi_{G}\right)_{x}=[s / 2]$ [35, Theorem 4.3.3]. Then

$$
\begin{aligned}
\mathrm{L}=\frac{1}{2}\left(\sum_{i=1}^{s} \hat{\eta}_{i}^{2}-\#\left\{j \mid \eta_{j} \text { is odd }\right\}\right)+2[s / 2]-3 n= \\
\quad \frac{1}{2}\left(\sum_{i=1}^{s}\left(\hat{\eta}_{i}-1\right)\left(\hat{\eta}_{i}-2\right)+3-\#\left\{j \mid \eta_{j} \text { is odd }\right\}-2(s-2[s / 2])\right) .
\end{aligned}
$$

If $\hat{\eta}_{1}=1$, then $\mathrm{L}=0$. This is the case of regular nilpotent elements.

If $\hat{\eta}_{1}=3$, then $\sum_{i=1}^{s}\left(\hat{\eta}_{i}-1\right)\left(\hat{\eta}_{i}-2\right)=2 \eta_{3} \geqslant 2$. Therefore $2 \mathrm{~L}=2 \eta_{2}+3-\#\{j \mid$ $\eta_{j}$ is odd $\}-2(s-2[s / 2])$. Since $\#\left\{j \mid \eta_{j}\right.$ is odd $\} \leqslant 3$ and $2(s-2[s / 2]) \leqslant 2,2 \mathrm{~L}$ is nonnegative. Furthermore, $\mathrm{L}=0$ if and only if $\eta_{3}=1$ and all $\eta_{i}$ 's are odd. If $\hat{\eta}_{1} \geqslant 5$, then $\sum_{i=1}^{s}\left(\hat{\eta}_{i}-1\right)\left(\hat{\eta}_{i}-2\right) \geqslant \hat{\eta}_{1}+2 \geqslant \#\left\{j \mid \eta_{j}\right.$ is odd $\}+2$. Next, $3-2(s-2[s / 2]) \geqslant 0$. Hence $\mathbf{L}$ is positive.

(C) $\mathfrak{g}=\mathfrak{s p}(\mathbb{V}), \operatorname{dim} \mathbb{V}=2 n$.

Here $\operatorname{dim}_{\mathfrak{z}}(x)=\frac{1}{2}\left(\sum_{i} \hat{\eta}_{i}^{2}+\#\left\{j \mid \eta_{j}\right.\right.$ is odd $\left.\}\right)$ and $\operatorname{rk}\left(d \pi_{G}\right)_{x}=[s / 2][35$, Theorem 4.3.3]. Then

$$
\begin{aligned}
\mathrm{L}=\frac{1}{2}\left(\sum_{i=1}^{s} \hat{\eta}_{i}^{2}+\right. & \left.\#\left\{j \mid \eta_{j} \text { is odd }\right\}\right)+2[s / 2]-3 n= \\
& \frac{1}{2}\left(\sum_{i=1}^{s}\left(\hat{\eta}_{i}-1\right)\left(\hat{\eta}_{i}-2\right)+\#\left\{j \mid \eta_{j} \text { is odd }\right\}-2(s-2[s / 2])\right) .
\end{aligned}
$$

It is easily seen that $L=0$ if and only if $\hat{\eta}_{1} \leqslant 2$. Otherwise it is positive.

(D) $\mathfrak{g}=\mathfrak{s o}(\mathbb{V}), \operatorname{dim} \mathbb{V}=2 n$.

Here $\hat{\eta}_{1}$ is even and $\operatorname{dim}_{\mathfrak{z} \mathfrak{g}}(x)$ is as in (B). For the rank of $d \pi_{G}$, we have [35, Theorem 4.4.2] $\operatorname{rk}\left(d \pi_{G}\right)_{x}=\left\{\begin{aligned} {[s / 2], } & \text { if } \hat{\eta}_{1} \geqslant 4 ; \\ (2 n-i+1) / 2, & \text { if } \boldsymbol{\eta}=(2 n-i, i) \text { with } i \text { odd; } \\ l, & \text { if } \boldsymbol{\eta}=(n, n) \text { and } n=2 l .\end{aligned}\right.$ 
Then

$$
\begin{aligned}
\mathrm{L}=\frac{1}{2}\left(\sum_{i=1}^{s} \hat{\eta}_{i}^{2}-\#\left\{j \mid \eta_{j} \text { is odd }\right\}\right)+2 \operatorname{rk}\left(d \pi_{G}\right)_{x}-3 n= \\
\frac{1}{2}\left(\sum_{i=1}^{s}\left(\hat{\eta}_{i}-1\right)\left(\hat{\eta}_{i}-2\right)+4 \operatorname{rk}\left(d \pi_{G}\right)_{x}-2 s-\#\left\{j \mid \eta_{j} \text { is odd }\right\}\right) .
\end{aligned}
$$

Now, a consideration of cases shows that $\mathrm{L}=0$ if $\hat{\eta}_{1}=2$. If $\hat{\eta}_{1} \geqslant 4$, then $\mathrm{L}>0$ unless $\boldsymbol{\eta}=\left(\eta_{1}, \eta_{2}, 1,1\right)$, where $\eta_{1}, \eta_{2}$ are both odd.

(EFG) $\mathfrak{g}$ is exceptional.

It is enough to check inequality (10.7) for sufficiently large orbits (with $\operatorname{dim} \mathfrak{z}_{\mathfrak{g}}(x)$ $\leqslant 3 \mathrm{rkg}$ ). To this end, one can consult the tables in [10, Ch. 8] for dimensions of orbits and [35, Appendix] for the values of $\operatorname{rk}\left(d \pi_{G}\right)_{x}$. Below we list all non-regular nilpotent orbits with $L=0$. The orbits are represented by their Dynkin-Bala-Carter labels.

$\mathbf{G}_{2}: \quad \mathbf{G}_{2}\left(a_{1}\right)$

$\mathbf{F}_{4}: \quad \mathbf{F}_{4}\left(a_{1}\right), \mathbf{F}_{4}\left(a_{2}\right) ;$

$\mathbf{E}_{6}: \quad \mathbf{E}_{6}\left(a_{1}\right), \mathbf{D}_{5}, \mathbf{E}_{6}\left(a_{3}\right) ;$

$\mathbf{E}_{7}: \quad \mathbf{E}_{7}\left(a_{1}\right), \mathbf{E}_{7}\left(a_{2}\right), \mathbf{E}_{6}, \mathbf{E}_{6}\left(a_{1}\right) ;$

$\mathbf{E}_{8}: \quad \mathbf{E}_{8}\left(a_{1}\right), \mathbf{E}_{8}\left(a_{2}\right), \mathbf{E}_{8}\left(a_{3}\right), \mathbf{E}_{8}\left(a_{4}\right)$.

Inspecting the tables in $[10$, Ch. 8$]$ shows that these are precisely the even nilpotent orbits whose weighted Dynkin diagrams have no adjacent zeros, which exactly means that $\left[\mathfrak{z}_{\mathfrak{g}}(h), \mathfrak{z}_{\mathfrak{g}}(h)\right]$ is a sum of several $\mathfrak{s l}_{2}$ 's.

For $\mathfrak{g}$ classical, there is a rule for writing out the characteristic $h$ in terms of $\boldsymbol{\eta}\left[39\right.$, Ch. IV]. Hence the Levi subalgebra $\mathfrak{z}_{\mathfrak{g}}(h)$ can be computed. This yields the last assertion of the theorem.

A geometric meaning of (10.7) will be made clear in the following result. Let $\mathfrak{g}=\mathfrak{g}_{0} \oplus \mathfrak{g}_{1}$ be a $\mathbb{Z}_{2}$-grading and $\vartheta$ the corresponding involutory automorphism of $\mathfrak{g}$. Then $\vartheta$ can be extended to an involution of the Takiff algebra $\hat{\mathfrak{g}}$ by letting $\vartheta(x+\epsilon y)=\vartheta(x)+\epsilon \vartheta(y)$. The corresponding eigenspaces are $\hat{\mathfrak{g}}_{0}=\mathfrak{g}_{0} \ltimes \mathfrak{g}_{0}$ and $\hat{\mathfrak{g}}_{1}=\mathfrak{g}_{1} \ltimes \mathfrak{g}_{1}$. Here $\hat{\mathfrak{g}}_{1}$ is a $\hat{\mathfrak{g}}_{0}$-module just in the sense of definition given in $\S 7$. The $G_{0}$-module $\mathfrak{g}_{1}$ is not extremely good, so that Theorem 10.2 cannot be applied. But it is 'good enough' in the sense that it satisfies properties (1), (2), (4) of Definition 10.1. 
10.8 Theorem. Suppose $\mathfrak{g}=\mathfrak{g}_{0} \oplus \mathfrak{g}_{1}$ is a $\mathbb{Z}_{2}$-grading of maximal rank, i.e., $\mathfrak{g}_{1}$ contains a Cartan subalgebra of $\mathfrak{g}$. Then the quotient morphism $\hat{\pi}$ : $\hat{\mathfrak{g}}_{1} \rightarrow \hat{\mathfrak{g}}_{1} / / \hat{G}_{0}$ is equidimensional.

Proof. Recall the relationship between orbits and null-cones for the actions $(G: \mathfrak{g})$ and $\left(G_{0}: \mathfrak{g}_{1}\right)$. The null-cones are $\mathcal{N}$ and $\mathcal{N}\left(\mathfrak{g}_{1}\right)$, respectively.

- $\mathcal{N}\left(\mathfrak{g}_{1}\right)=\mathcal{N} \cap \mathfrak{g}_{1} ;$

- $G \cdot x \cap \mathfrak{g}_{1}$ is a union of finitely many $G_{0}$-orbits;

- If $x \in \mathfrak{g}_{1}$, then $\operatorname{dim} G_{0} \cdot x=\frac{1}{2} \operatorname{dim} G \cdot x$;

- For any $x \in \mathfrak{g}$, we have $G \cdot x \cap \mathfrak{g}_{1} \neq \varnothing$;

- $\mathbb{k}[\mathfrak{g}]^{G} \simeq \mathbb{k}\left[\mathfrak{g}_{1}\right]^{G_{0}}$.

The first three properties hold for all $\mathbb{Z}_{2}$-gradings, whereas the last two are characteristic for the involutions of maximal rank, see [1].

Let us see what the equidimensionality criterion (Theorem 8.10(i)) means here. We have $V=\mathfrak{g}_{1}, G=G_{0}$, and $m=\operatorname{dim} \mathfrak{g}_{1} / / G_{0}$. Since $\mathcal{N}\left(\mathfrak{g}_{1}\right)$ consists of finitely many $G_{0}$-orbits, that criterion reads

$$
\operatorname{dim} \mathcal{N}\left(\mathfrak{g}_{1}\right)-\operatorname{dim} G_{0} \cdot x \geqslant \operatorname{dim} \mathfrak{g}_{1} / / G_{0}-\operatorname{rk}\left(d \pi_{G_{0}}\right)_{x}
$$

for any $x \in \mathcal{N}\left(\mathfrak{g}_{1}\right)$. Here $\pi_{G_{0}}: \mathfrak{g}_{1} \rightarrow \mathfrak{g}_{1} / / G_{0}$ is the quotient morphism. In view of the above properties of such $\mathbb{Z}_{2}$-gradings, we have $\operatorname{dim} \mathcal{N}\left(\mathfrak{g}_{1}\right)=\frac{1}{2} \operatorname{dim} \mathcal{N}=$ $\frac{1}{2}(\operatorname{dim} \mathfrak{g}-\mathrm{rk} \mathfrak{g}), \operatorname{dim} \mathfrak{g}_{1} / / G_{0}=\mathrm{rk} \mathfrak{g}$, and $\operatorname{rk}\left(d \pi_{G_{0}}\right)_{x}=\operatorname{rk}\left(d \pi_{G}\right)_{x}$. The latter stems from the isomorphism $\mathbb{k}[\mathfrak{g}]^{G} \simeq \mathbb{k}\left[\mathfrak{g}_{1}\right]^{G_{0}}$. Rewriting the previous inequality using this data yields precisely inequality (10.7)! Thus, the fact that $\hat{\pi}$ is equidimensional is essentially equivalent to Theorem 10.6.

Yet another geometric application of Eq. (10.7) is the following (cf. Theorem 9.14):

10.9 Theorem. Set $\mathfrak{g}^{[n]}=\mathfrak{g} \ltimes \mathfrak{g}^{\oplus n}$, where $\mathfrak{g}^{\oplus n}$ (the sum of $n$ copies) regarded as commutative Lie algebra and $n \geqslant 1$. Consider the adjoint action $\left(G^{[n]}: \mathfrak{g}^{[n]}\right)$. Then $\pi_{G^{[n]}}$ is equidimensional if and only if $n \leqslant 2$.

Proof. For $n=1$, the assertion is already proved. Next, $\operatorname{dim}\left(\mathfrak{g}^{\oplus n}\right)^{T}=$ $n \mathrm{rk} \mathfrak{g}$ and for $x \in \mathcal{N}$ the equidimensionality condition of Theorem 8.1(i) reads $\operatorname{dim} \mathfrak{z}_{\mathfrak{g}}(x)-\mathrm{rk} \mathfrak{g} \geqslant n\left(\operatorname{rk} \mathfrak{g}-\operatorname{rk}\left(d \pi_{G}\right)_{x}\right)$, which is exactly $(10.7)$ for $n=2$. 
Conversely, if $n \geqslant 3$, then this condition is not satisfied for the subregular nilpotent orbit.

Remark. In the last theorem, the null-cone $\mathcal{N}\left(\mathfrak{g}^{[2]}\right)$ is always reducible. Indeed, each nilpotent $G$-orbit such that $\mathrm{L}=0$ in (10.7) gives rise to an irreducible component of $\mathcal{N}\left(\mathfrak{g}^{[2]}\right)$, see Remark 8.3(1). The proof of Theorem 10.6 shows that, for any $\mathfrak{g}$, there are at least two orbits with $L=0$.

There are several equivalent ways to present inequality (10.7). Let $\mathcal{B}$ denote the variety of Borel subgroups of $G$. For any $x \in \mathcal{N}$, set $\mathcal{B}_{x}=\left\{B^{\prime} \in \mathcal{B} \mid x \in\right.$ Lie $\left.B^{\prime}\right\}$. Recall that $\mathfrak{X}_{i}=\mathfrak{X}_{i, \mathfrak{g}}=\left\{x \in \mathfrak{g} \mid \operatorname{rk}\left(d \pi_{G}\right)_{x}=i\right\}$ and $\mathfrak{X}_{i, \mathfrak{g}}(\mathcal{N})=\mathfrak{X}_{i, \mathfrak{g}} \cap \mathcal{N}$. This stratification is determined by the covariants of type $\mathfrak{g}$.

10.10 Proposition. Let $\mathfrak{g}$ be a simple Lie algebra and $m=\mathrm{rk} \mathfrak{g}$. Then the following holds:

(1) $\operatorname{codim}_{\mathcal{N}} \mathfrak{X}_{i, \mathfrak{g}}(\mathcal{N}) \geqslant 2(m-i)$ for any $i=0,1, \ldots, m$;

(2) $\operatorname{dim} \mathcal{B}_{x}+\mathrm{rk}\left(d \pi_{G}\right)_{x} \geqslant \operatorname{rk} \mathfrak{g}$ for any $x \in \mathcal{N}$;

(3) If $\mathcal{O}$ is the local ring of any $G$-orbit in $\mathcal{N}$, then $\operatorname{edim} \mathcal{O} \leqslant \frac{3}{2} \operatorname{dim} \mathcal{O}$;

(4) If $\mathfrak{g}=\mathfrak{g}_{0} \oplus \mathfrak{g}_{1}$ is a $\mathbb{Z}_{2}$-grading of maximal rank and $x \in \mathcal{N}\left(\mathfrak{g}_{1}\right)$, then $\operatorname{dim}\left(G_{0}\right)_{x}+\operatorname{rk}\left(d \pi_{G_{0}}\right)_{x} \geqslant \operatorname{rkg}$.

(5) If $\mathfrak{g}=\mathfrak{g}_{0} \oplus \mathfrak{g}_{1}$ is a $\mathbb{Z}_{2}$-grading of maximal rank and $\mathcal{O}^{\prime}$ is the local ring of a $G_{0}$-orbit in $\mathcal{N}\left(\mathfrak{g}_{1}\right)$, then $\operatorname{edim} \mathcal{O}^{\prime} \leqslant 2 \operatorname{dim} \mathcal{O}^{\prime}$.

Proof. In fact, all these conditions are equivalent to inequality (10.7). Since $\mathcal{N}$ contains finitely many $G$-orbits, (1) can be written as $\operatorname{codim}_{\mathcal{N}} G \cdot x \geqslant$ $2\left(m-\mathrm{rk}\left(\mathrm{d} \pi_{G}\right)_{x}\right)$ for any $x \in \mathcal{N}$, which makes it clear that (1) is equivalent to $(10.7)$. For $(2)$, one should use the fact that $\operatorname{dim} \mathcal{B}_{x}=\frac{1}{2}\left(\operatorname{dim} \mathfrak{z}_{\mathfrak{g}}(x)-\operatorname{rk} \mathfrak{g}\right)$, see e.g. $[38,4.3 .10,4.5]$. For $(3)$, one have to use formulae for $\operatorname{dim} \mathcal{O}$ and $\operatorname{edim} \mathcal{O}$ written out in the proof of Theorem 10.2. For (4), we notice that since $\operatorname{dim} \mathfrak{g}_{1}-\operatorname{dim} \mathfrak{g}_{0}=\mathrm{rkg}$, the equality $\operatorname{dim} G_{0} \cdot x=\frac{1}{2} \operatorname{dim} G \cdot x$ is equivalent to that $\operatorname{dim}\left(G_{0}\right)_{x}=\frac{1}{2}\left(\operatorname{dim} \mathfrak{z}_{\mathfrak{g}}(x)-\mathrm{rk} \mathfrak{g}\right)=\operatorname{dim} \mathcal{B}_{x}$. Finally, the inequalities in (4) and (5) are obtained from each other via simple transformations.

Remark. Concerning (5), we note that this inequality is weaker than the Goto-Watanabe inequality from the proof of Theorem 10.2 , but $\mathcal{N}\left(\mathfrak{g}_{1}\right)$ is not normal and can be reducible.

10.11 Corollary. $\operatorname{codim}_{\mathfrak{g}} \mathfrak{X}_{i, \mathfrak{g}} \geqslant 3(m-i)$ for any $i=0,1, \ldots, m$.

Proof. It follows from the definition of $\mathfrak{X}_{i, \mathfrak{g}}$ that $\operatorname{dim} \overline{\mathfrak{X}}_{i, \mathfrak{g}} / / G=i$. Since $\overline{\mathfrak{X}}_{i, \mathfrak{g}}$ is conical, the fibre of the origin of the morphism $\overline{\mathfrak{X}}_{i, \mathfrak{g}} \rightarrow \overline{\mathfrak{X}}_{i, \mathfrak{g}} / / G$ has the 
maximal dimension, i.e., $\operatorname{dim} \mathfrak{X}_{i, \mathfrak{g}} \leqslant i+\operatorname{dim} \mathfrak{X}_{i, \mathfrak{g}}(\mathcal{N})$, which is exactly what we want, in view of Proposition 10.10(1).

There are many open problems and observations related to our results on reductive Takiff algebras and $\mathbb{Z}_{2}$-contractions. Here are some of them.

$1^{\circ}$. It seems that if $H$ is a spherical reductive subgroup of $G$ and $\mathfrak{k}=\mathfrak{h} \ltimes \mathfrak{m}$ is the corresponding isotropy contraction of $\mathfrak{g}$, then $\pi_{K}$ is always equidimensional. At least, I have verified this in case $G$ is simple. In fact, Examples 9.6 and 9.15 present several instances of this verification.

$2^{o}$. It would be quite interesting to have a case-free proof for Theorem 10.6 or, equivalently, 10.8. Various equivalent forms of that result presented in Proposition 10.10 suggest that there might be different approaches to proving it. From the geometric point of view, the equidimensionality of $\hat{\pi}$ means that there exists a transversal subspace to $\mathcal{N}\left(\hat{\mathfrak{g}}_{1}\right)$, i.e., a subspace $U$ such that $\operatorname{dim} U=$ $\operatorname{dim} \hat{\mathfrak{g}}_{1} / / \hat{G}_{0}$ and $U \cap \mathcal{N}\left(\hat{\mathfrak{g}}_{1}\right)=\{0\}$.

$3^{\circ}$. Whenever some quotient morphism is equidimensional, it is interesting to find a natural transversal subspace to the null-cone. One may ask for such a subspace in the setting of Theorems 9.13, 10.2, 10.8. Even for the adjoint representation of $\hat{\mathfrak{g}}=\mathfrak{g} \ltimes \mathfrak{g}$ it is not known how to naturally construct a transversal space to $\mathcal{N}(\hat{\mathfrak{g}})$. If $\Delta_{\mathfrak{t}} \subset \mathfrak{g} \ltimes \mathfrak{g}$ is the diagonally embedded Cartan subalgebra, then $\Delta_{\mathfrak{t}} \cap \mathcal{N}(\hat{\mathfrak{g}})=\{0\}$, so that one has a "one-half" of a transversal space. The problem is to construct the second half. Similarly, if $\mathfrak{k}$ is a $\mathbb{Z}_{2}$-contraction of a simple Lie algebra, it is not known how to construct a transversal space to $\mathcal{N}^{u}(\mathfrak{k})$.

$4^{o}$. If one knows that some null-cone $\mathcal{N}$ is irreducible, then it is tempting to find a resolution of singularities for $\mathcal{N}$.

$5^{o}$. A case-by-case verification shows that $\mathfrak{X}_{1, \mathfrak{g}}(\mathcal{N})$ is irreducible for any simple $\mathfrak{g}$, and the dense $G$-orbit in it is Richardson.

\section{§11. On Invariants and Null-Cones for Generalised Takiff Lie Algebras}

Following [33], we recall the definition of a generalised Takiff Lie algebra. The infinite-dimensional $\mathbb{k}$-vector space $\mathfrak{q}_{\infty}:=\mathfrak{q} \otimes \mathbb{k}[\mathrm{T}]$ has a natural structure of a Lie algebra such that $\left[x \otimes \mathrm{T}^{l}, y \otimes \mathrm{T}^{k}\right]=[x, y] \otimes \mathrm{T}^{l+k}$. Then $\mathfrak{q}_{\geqslant(n+1)}=$ $\bigoplus_{j \geqslant n+1} \mathfrak{q} \otimes \mathrm{T}^{j}$ is an ideal of $\mathfrak{q}_{\infty}$, and the respective quotient is a generalised Takiff Lie algebra, denoted $\mathfrak{q}\langle n\rangle$. We also say that $\mathfrak{q}\langle n\rangle$ is the $n$-th Takiff algebra. Write $Q\langle n\rangle$ for the corresponding connected group. Clearly, $\operatorname{dim} \mathfrak{q}\langle n\rangle=(n+1) \operatorname{dim} \mathfrak{q}$ and $\mathfrak{q}\langle 1\rangle \simeq \mathfrak{q} \ltimes \mathfrak{q}$. The main results of [33] are the following: 
(i) ind $\mathfrak{q}\langle n\rangle=(n+1)$ ind $\mathfrak{q}$,

(ii) if $\mathfrak{q}=\mathfrak{g}$ is semisimple, then $\mathbb{k}[\mathfrak{g}\langle n\rangle]^{G\langle n\rangle}$ is a polynomial algebra whose set of basic invariants is explicitly described.

Actually, the authors of [33] work with invariants of the coadjoint representation of $G\langle n\rangle$, but this makes no difference, since $\mathfrak{g}\langle n\rangle$ is quadratic.

In this section, we generalise the results from (ii) in the spirit of $\S 7$. Let $\mathfrak{q}\langle n\rangle^{u}$ denote the image of $\mathfrak{q}_{\geqslant 1}$ in $\mathfrak{q}\langle n\rangle$. It is a nilpotent Lie algebra, which is noncommutative for $n \geqslant 2$, and $\mathfrak{q}\langle n\rangle \simeq \mathfrak{q} \ltimes \mathfrak{q}\langle n\rangle^{u}$. Accordingly, one obtains the semi-direct product structure of the group: $Q\langle n\rangle=Q \ltimes Q\langle n\rangle^{u}$.

11.1 Theorem. Suppose $\mathfrak{q}$ satisfies conditions

(1) $\mathbb{k}[\mathfrak{q}]^{Q}$ is a polynomial algebra;

(2) $\max \operatorname{dim}_{x \in \mathfrak{q}} Q \cdot x=\operatorname{dim} \mathfrak{q}-\operatorname{dim} \mathfrak{q} / / Q$;

(3) If $\pi_{Q}: \mathfrak{q} \rightarrow \mathfrak{q} / / Q$ is the quotient morphism and $\Omega:=\{x \in \mathfrak{q} \mid$ $\left(d \pi_{Q}\right)_{x}$ is onto $\}$, then $\mathfrak{q} \backslash \Omega$ contains no divisors.

Then

(i) $\mathbb{k}[\mathfrak{q}\langle n\rangle]^{Q\langle n\rangle^{u}}$ is a polynomial algebra of Krull dimension $\operatorname{dim} \mathfrak{q}+n \operatorname{dim} \mathfrak{q} / / Q$ whose algebraically independent generators can explicitly be described;

(ii) $\mathbb{k}[\mathfrak{q}\langle n\rangle]^{Q\langle n\rangle}$ is a polynomial algebra of Krull dimension $(n+1) \operatorname{dim} \mathfrak{q} / / Q$ whose algebraically independent generators can explicitly be described.

Proof. Let $\boldsymbol{x}=x_{0}+\epsilon x_{1}+\cdots+\epsilon^{n} x_{n}$ denote the image of $\sum_{i=0}^{n} x_{i} \otimes \mathrm{T}^{i}$ in $\mathfrak{q}\langle n\rangle$. Here each $x_{i} \in \mathfrak{q}$ and $\epsilon$ is regarded as the image of $\mathrm{T}$ in $\mathbb{k}[\mathrm{T}] /\left(\mathrm{T}^{n+1}\right)$. Set $m=\operatorname{dim} \mathfrak{q} / / Q$, and let $f_{1}, \ldots, f_{m}$ be a set of basic invariants in $\mathbb{k}[\mathfrak{q}]^{Q}$. Expand the polynomial $f_{i}\left(x_{0}+\epsilon x_{1}+\cdots+\epsilon^{n} x_{n}\right)$ using the relation $\epsilon^{n+1}=0$. We obtain

$$
f_{i}\left(x_{0}+\epsilon x_{1}+\cdots+\epsilon^{n} x_{n}\right)=\sum_{j=0}^{n} \epsilon^{j} \widehat{F}_{i}^{(j)}\left(x_{0}, x_{1}, \ldots, x_{n}\right) .
$$

Following the argument in [33, Sect. III], one proves that

$$
\begin{gathered}
\widehat{F}_{i}^{(j)} \text { depends only on } x_{0}, \ldots, x_{j} \text { and } \\
\widehat{F}_{i}^{(j)}\left(x_{0}, \ldots, x_{j}\right)=\left\langle\left(d f_{i}\right)_{x_{0}}, x_{j}\right\rangle+p_{i j}\left(x_{0}, \ldots, x_{j-1}\right) .
\end{gathered}
$$

It follows from the construction that all $\widehat{F}_{i}^{(j)}$ belong to $\mathbb{k}[\mathfrak{q}\langle n\rangle]^{Q\langle n\rangle}$.

(i) Making use of Lemma 6.1 and Eq. (11.2), we prove that the polynomials $\widehat{F}_{i}^{(j)}, i=1, \ldots, m, j=1, \ldots, n$, and the coordinates on the first factor in $\mathfrak{q}\langle n\rangle$ freely generate $\mathbb{k}[\mathfrak{q}\langle n\rangle]^{Q\langle n\rangle^{u}}$. 
Consider the mapping

$$
\psi: \mathfrak{q}\langle n\rangle \rightarrow \mathfrak{q} \times \mathbb{k}^{n m},
$$

given by $\psi(\boldsymbol{x})=\left(x_{0}, \widehat{F}_{1}^{(1)}(\boldsymbol{x}), \ldots, \widehat{F}_{m}^{(n)}(\boldsymbol{x})\right)$. Here we regard $\mathfrak{q}$ as $\mathfrak{q}\langle n\rangle / \mathfrak{q}\langle n\rangle^{u}$, so that $\mathfrak{q} \times \mathbb{k}^{n m}$ is a variety with trivial $Q\langle n\rangle^{u}$-action. If $x_{0} \in \Omega$, then $\left(d f_{i}\right)_{x_{0}}$ are linearly independent. Therefore Eq. (11.2) shows that the system $\widehat{F}_{i}^{(j)}\left(x_{0}+\epsilon y_{1}+\cdots+\epsilon^{n} y_{n}\right)=\alpha_{i}^{(j)}, i=1, \ldots, m, j=1, \ldots, n$ has a solution, say $\left(y_{1}, \ldots, y_{n}\right)$, for any $n m$-tuple $\boldsymbol{\alpha}=\left(\alpha_{1}^{(1)}, \ldots, \alpha_{m}^{(n)}\right)$. Indeed, $\left(y_{1}, \ldots, y_{n}\right)$ can be computed consecutively: First $y_{1}$, then $y_{2}$, and so on. Hence $\operatorname{Im} \psi \supset \Omega \times \mathbb{k}^{n m}$, i.e., $\operatorname{Im} \psi$ contains a big open subset of $\mathfrak{q} \times \mathbb{k}^{n m}$. This also implies that the coordinates on $\mathfrak{q}$ and the polynomials $\widehat{F}_{i}^{(j)}$ are algebraically independent. It follows that

$$
\max _{\boldsymbol{x} \in \mathfrak{q}\langle n\rangle} \operatorname{dim} Q\langle n\rangle^{u} \cdot \boldsymbol{x} \leqslant \operatorname{dim} \mathfrak{q}\langle n\rangle-\operatorname{dim} \mathfrak{q}-m n=n(\operatorname{dim} \mathfrak{q}-m) .
$$

Next, consider $\Omega^{\prime}=\Omega \cap\{z \in \mathfrak{q} \mid \operatorname{dim} Q \cdot z=\operatorname{dim} \mathfrak{q}-m\}$. In view of condition (2), it is still a non-empty open $Q$-stable subset of $\mathfrak{q}$. Fix $x_{0} \in \Omega^{\prime}$, and let $\left(\bar{y}_{1}, \ldots, \bar{y}_{n}\right)$ be a solution to the system $\widehat{F}_{i}^{(j)}\left(x_{0}+\epsilon y_{1}+\cdots+\epsilon^{n} y_{n}\right)=\alpha_{i}^{(j)}, i=1, \ldots, m$, $j=1, \ldots, n$. Then $\psi^{-1}\left(x_{0}, \boldsymbol{\alpha}\right) \supset Q\langle n\rangle^{u} \cdot\left(x_{0}+\sum_{i=1}^{n} \epsilon^{i} \bar{y}_{i}\right)$. Since $x_{0} \in \Omega$, we have $\operatorname{dim} \psi^{-1}\left(x_{0}, \boldsymbol{\alpha}\right)=n(\operatorname{dim} \mathfrak{q}-m)$. On the other hand, the following holds

Claim. If $x \in \mathfrak{q}^{r e g}$, then $\operatorname{dim} Q\langle n\rangle^{u} \cdot\left(x+\epsilon y_{1}+\cdots+\epsilon^{n} y_{n}\right)=n \operatorname{dim} Q \cdot x=$ $n(\operatorname{dim} \mathfrak{q}-m)$ for any $\left(y_{1}, \ldots, y_{n}\right) \in \mathfrak{q}^{n}$.

Proof of the claim. We argue by induction on $n$. For $n=1$, the assertion is obvious. Assume that $n \geqslant 2$. Consider the $Q\langle n\rangle^{u}$-equivariant projection

$$
\left(x+\sum_{1}^{n} \epsilon^{i} y_{i} \in \mathfrak{q}\langle n\rangle\right) \stackrel{p}{\longmapsto}\left(x+\sum_{1}^{n-1} \epsilon^{i} y_{i} \in \mathfrak{q}\langle n-1\rangle\right) .
$$

Let $\mathcal{O}_{n}$ denote the $Q\langle n\rangle^{u}$-orbit of $x+\sum_{1}^{n} \epsilon^{i} y_{i}$. Then $p\left(\mathcal{O}_{n}\right)=\mathcal{O}_{n-1}$. By the induction hypothesis, $\operatorname{dim} \mathcal{O}_{n-1}=(n-1)(\operatorname{dim} \mathfrak{q}-m)$. It is easily seen that

$$
p^{-1}\left(x+\sum_{1}^{n-1} \epsilon^{i} y_{i}\right) \cap \mathcal{O}_{n} \supset x+\sum_{1}^{n-1} \epsilon^{i} y_{i}+\epsilon^{n}\left(y_{n}+[\mathfrak{q}, x]\right) .
$$

For, the right hand side is precisely an orbit of the subgroup $\exp \left(\epsilon^{n} \mathfrak{q}\right) \subset Q\langle n\rangle^{u}$. Hence $\operatorname{dim} \mathcal{O}_{n} \geqslant n(\operatorname{dim} \mathfrak{q}-m)$. But it is already proved that the dimension of every $Q\langle n\rangle^{u}$-orbit is at most $n(\operatorname{dim} \mathfrak{q}-m)$.

Hence $\psi^{-1}\left(x_{0}, \boldsymbol{\alpha}\right)=Q\langle n\rangle^{u} \cdot\left(x_{0}+\epsilon \bar{y}_{1}+\cdots+\epsilon^{n} \bar{y}_{n}\right)$ for dimension reason. Thus, a generic fibre of $\psi$ is an $Q\langle n\rangle^{u}$-orbit, and Lemma 6.1 applies here. 
(ii) Follows from (i) and the description of $Q\langle n\rangle^{u}$-invariants.

11.3 Remark. It was noticed in $\S 9$ that any Takiff algebra $\mathfrak{q} \ltimes \mathfrak{q}$ is a $\mathbb{Z}_{2}$-contraction of $\mathfrak{q} \dot{+} \mathfrak{q}$. Similar phenomenon holds for the generalised Takiff algebras: $\mathfrak{q}\langle n\rangle$ is a contraction of $\mathfrak{q} \dot{+} \cdots \dot{+} \mathfrak{q}=(n+1) \mathfrak{q}$. The starting point for constructing such a contraction is to consider the action $\mathbb{Z}_{n+1}$ on $(n+1) \mathfrak{q}$ that cyclically permutes the summands. On the other hand, given $\mathfrak{q}\langle n\rangle$, it can further be contracted to $\mathfrak{q} \ltimes \mathfrak{q}^{\oplus n}$, the "usual" semi-direct product, where $\mathfrak{q}^{\oplus n}$ is regarded as commutative Lie algebra. The details are left to the reader. Thus,

$$
\mathfrak{q} \dot{+} \cdots \dot{+} \mathfrak{q}=(n+1) \mathfrak{q} \rightsquigarrow \mathfrak{q}\langle n\rangle \rightsquigarrow \mathfrak{q} \ltimes \mathfrak{q}^{\oplus n}
$$

is a chain of contractions.

Using Eq. (10.7) and Eq. (11.2) one can easily prove that if $\mathfrak{g}$ is semisimple and $\mathfrak{g}\langle 2\rangle$ is the second Takiff Lie algebra, then the quotient morphism $\pi_{G\langle 2\rangle}$ : $\mathfrak{g}\langle 2\rangle \rightarrow \mathfrak{g}\langle 2\rangle / / G\langle 2\rangle$ is equidimensional. This is a particular case of the theorem of Eisenbud-Frenkel mentioned in the introduction.

\section{Acknowledgements}

Work on this article commenced during my visits to the Université de Poitiers (France) in 1996-98. I would like to thank Thierry Levasseur for arranging those visits, inspiring conversations, and drawing my attention to work of Geoffriau. Thanks are also due to Michel Brion for sharing some important insights on Takiff algebras. I am grateful to Sasha Premet for drawing my attention to results of Eisenbud and Frenkel.

\section{References}

[1] L. V. Antonyan, Classification of homogeneous elements of $\mathbb{Z}_{2}$-graded semisimple Lie algebras, Vestnik Moskov. Univ. Ser. I Mat. Mekh. 1982, no. 2, 29-34 (Russian). English translation: Moscow Univ. Math. Bulletin 37 (1982), no. 2, 36-43.

[2] L. L. Avramov, Complete intersections and symmetric algebras, J. Algebra 73 (1981), no. 1, 248-263.

[3] M. Brion. Sur la théorie des invariants, Publ. Math. Univ. Pierre et Marie Curie 45 (1981), 1-92.

[4] Inst. Fourier (Grenoble) 33 (1983), no. 1, 1-27.

[5] - Invariants et covariants des groupes algébriques réductifs, Dans: "Théorie des invariants et géometrie des variétés quotients" (Travaux en cours, t. 61), 83-168, Paris: Hermann, 2000.

[6] 
[7] B. Broer, Line bundles on the cotangent bundle of the flag variety, Invent. Math. 113 (1993), no. 1, 1-20.

[8] R. K. Brylinski, Limits of weight spaces, Lusztig's $q$-analogs, and fiberings of adjoint orbits, J. Amer. Math. Soc. 2 (1989), no. 3, 517-533.

[9] R. Brylinski and B. Kostant, The variety of all invariant symplectic structures on a homogeneous space and normalizers of isotropy subgroups, in Symplectic geometry and mathematical physics (Aix-en-Provence, 1990), 80-113, Progr. Math., 99, Birkhäuser, Boston.

[10] D. H. Collingwood and W. M. McGovern, Nilpotent orbits in semisimple Lie algebras, Van Nostrand Reinhold, New York, 1993.

[11] J. Dadok and V. Kac, Polar representations, J. Algebra 92 (1985), no. 2, 504-524.

[12] I. V. Dolgachev, Rationality of fields of invariants, in Algebraic geometry, Bowdoin, 1985 (Brunswick, Maine, 1985), 3-16, Proc. Sympos. Pure Math., Part 2, Amer. Math. Soc., Providence, RI.

[13] M. Duflo and M. Vergne, Une propriété de la représentation coadjointe d'une algèbre de Lie, C. R. Acad. Sci. Paris Sér. A-B 268 (1969), A583-A585.

[14] A. G. Èlašvili, Canonical form and stationary subalgebras of points in general position for simple linear Lie groups, Funkcional. Anal. i Priložen. 6 (1972), no. 1, 51-62 (Russian). English translation: Funct. Anal. Appl. 6 (1972), 44-53.

[15] - Frobenius Lie algebras, Funktsional. Anal. i Prilozhen. 16 (1982), no. 4, 94-95 (Russian). English translation: Funct. Anal. Appl. 16 (1982), 326-328.

[16] F. Geoffriau, Sur le centre de l'algèbre enveloppante d'une algèbre de Takiff, Ann. Math. Blaise Pascal 1 (1994), no. 2, 15-31.

[17] W. Hesselink, Singularities in the nilpotent scheme of a classical group, Trans. Amer. Math. Soc. 222 (1976), 1-32.

[18] Cohomology and the resolution of the nilpotent variety, Math. Ann. 223 (1976), no. 3, 249-252.

[19] J. Igusa, Geometry of absolutely admissible representations, in Number theory, algebraic geometry and commutative algebra, in honor of Yasuo Akizuki, 373-452, Kinokuniya, Tokyo, 1973.

[20] F. Knop, Über die Glattheit von Quotientenabbildungen, Manuscripta Math. 56 (1986), no. 4, 419-427.

[21] B. Kostant, Lie group representations on polynomial rings, Amer. J. Math. 85 (1963), 327-404.

[22] T. Levasseur and J. T. Stafford, Differential operators commuting with invariant functions, Comment. Math. Helv. 72 (1997), no. 3, 426-433.

[23] D. Luna, Sur les orbites fermées des groupes algébriques réductifs, Invent. Math. 16 (1972), 1-5.

[24] M. Mustață, Jet schemes of locally complete intersection canonical singularities, (with an appendix by D. Eisenbud and E. Frenkel), Invent. Math. 145 (2001), no. 3, 397-424.

[25] H. Nakajima and K. Watanabe, The classification of quotient singularities which are complete intersections, in Complete intersections (Acireale, 1983), 102-120, Lecture Notes in Math., 1092, Springer, Berlin.

[26] D. I. Panyushev, Orbit spaces of finite and connected linear groups, Izv. Akad. Nauk SSSR Ser. Mat. 46 (1982), no. 1, 95-99 (Russian). English translation: Math. USSRIzv. 20 (1983), 97-101.

[27] commuting varieties, Compositio Math. 94 (1994), no. 2, 181-199.

[28] Complexity and rank of actions in invariant theory, J. Math. Sci. (New York) 95 (1999), no. 1, 1925-1985.

[29] $ـ$ On covariants of reductive algebraic groups, Indag. Math. (N.S.) 13 (2002), no. 1, 125-129.

[30] normalizer of the centralizer, Math. Proc. Cambridge Philos. Soc. 134 (2003), no. 1, 
41-59.

[31] An extension of Raïs' theorem and seaweed subalgebras of simple Lie algebras, Ann. Inst. Fourier (Grenoble) 55 (2005), no. 3, 693-715.

[32] M. Raïs, L'indice des produits semi-directs $E \times_{\rho} \mathfrak{g}$, C. R. Acad. Sci. Paris Sér. A-B 287 (1978), no. 4, A195-A197.

[33] M. Raïs and P. Tauvel, Indice et polynômes invariants pour certaines algèbres de Lie, J. Reine Angew. Math. 425 (1992), 123-140.

[34] R. W. Richardson, Jr., Principal orbit types for algebraic transformation spaces in characteristic zero, Invent. Math. 16 (1972), 6-14.

[35] Derivatives of invariant polynomials on a semisimple Lie algebra, in Miniconference on harmonic analysis and operator algebras (Canberra, 1987), 228-241, Austral. Nat. Univ., Canberra.

[36] G. W. Schwarz, Representations of simple Lie groups with regular rings of invariants, Invent. Math. 49 (1978), no. 2, 167-191.

[37] J.-P. Serre, Complex semisimple Lie algebras, Translated from the French by G. A. Jones, Springer, New York, 1987.

[38] T. A. Springer, Conjugacy classes in algebraic groups, in Group theory, Beijing 1984, 175-209, Lecture Notes in Math., 1185, Springer, Berlin.

[39] T. A. Springer and R. Steinberg, Conjugacy classes, in Seminar on Algebraic Groups and Related Finite Groups (The Institute for Advanced Study, Princeton, N.J., 1968/69), 167-266, Lecture Notes in Math., 131, Springer, Berlin.

[40] S. J. Takiff, Rings of invariant polynomials for a class of Lie algebras, Trans. Amer. Math. Soc. 160 (1971), 249-262.

[41] P. Tauvel and R. W. T. Yu, Indice et formes linéaires stables dans les algèbres de Lie, J. Algebra 273 (2004), no. 2, 507-516.

[42] M. Van den Bergh, Modules of covariants, in Proceedings of the International Congress of Mathematicians, Vol. 1, 2 (Zürich, 1994), 352-362, Birkhäuser, Basel.

[43] W. V. Vasconcelos, Arithmetic of blowup algebras, Cambridge Univ. Press, Cambridge, 1994.

[44] E. B. Vinberg, On stability of actions of reductive algebraic groups. Fong, Yuen (ed.) et al., "Lie algebras, rings and related topics". Papers of the 2nd Tainan-Moscow international algebra workshop, Tainan, Taiwan, January 11-17, 1997. Hong Kong: Springer, 188-202 (2000).

[45] E. B. Vinberg, V. V. Gorbatsevich and A. L. Onishchik, Sovremennye problemy matematiki. Fundamentalnye napravleniya, t. 41.Moskva: VINITI 1990 (Russian). English translation: Lie Groups and Lie Algebras III (Encyclopaedia Math. Sci., vol. 41) Berlin: Springer 1994.

[46] È. B. Vinberg and V. L. Popov, Invariant theory, in Algebraic geometry, 4 (Russian), 137-314, 315, Akad. Nauk SSSR, Vsesoyuz. Inst. Nauchn. i Tekhn. Inform., Moscow: VINITI 1989 (Russian). English translation in: Algebraic Geometry IV (Encyclopaedia Math. Sci., vol. 55, pp.123-284) Berlin: Springer 1994.

[47] T. Vust, Covariants de groupes algébriques réductifs, Thèse no. 1671, Université de Genève, 1974.

[48] O. S. Yakimova, The index of centralisers of elements in classical Lie algebras, Funktsional. Anal. i Prilozhen. 40 (2006), no. 1, 52-64, 96; translation in Funct. Anal. Appl. 40 (2006), no. 1, 42-51. 